\title{
WestVirginiaUniversity
}

THE RESEARCH REPOSITORY @ WVU

Graduate Theses, Dissertations, and Problem Reports

2008

\section{Hemodynamics of intracranial aneurysms: Factors that may influence occurrence}

Francisco Adolfo Pino Romainville

West Virginia University

Follow this and additional works at: https://researchrepository.wvu.edu/etd

\section{Recommended Citation}

Pino Romainville, Francisco Adolfo, "Hemodynamics of intracranial aneurysms: Factors that may influence occurrence" (2008). Graduate Theses, Dissertations, and Problem Reports. 3483.

https://researchrepository.wvu.edu/etd/3483

This Dissertation is protected by copyright and/or related rights. It has been brought to you by the The Research Repository @ WVU with permission from the rights-holder(s). You are free to use this Dissertation in any way that is permitted by the copyright and related rights legislation that applies to your use. For other uses you must obtain permission from the rights-holder(s) directly, unless additional rights are indicated by a Creative Commons license in the record and/ or on the work itself. This Dissertation has been accepted for inclusion in WVU Graduate Theses, Dissertations, and Problem Reports collection by an authorized administrator of The Research Repository @ WVU.

For more information, please contact researchrepository@mail.wvu.edu. 


\title{
HEMODYNAMICS OF INTRACRANIAL ANEURYSMS: FACTORS
} THAT MAY INFLUENCE OCCURRENCE

\author{
Francisco Adolfo Pino Romainville
}

Dissertation submitted to the

College of Engineering and Mineral Resources

at West Virginia University

in partial fulfillment of the requirements

for the degree of

Doctor of Philosophy

in

Mechanical Engineering

Ismail B. Celik, Ph.D., Chair

David J. Klinke, Ph.D.

Wade W. Huebsch, Ph.D.

Osama Mukdadi, Ph.D.

Ansaar T. Rai, MD

Department of Mechanical and Aerospace Engineering

Morgantown, West Virginia

2008

Keywords: biological flows, intracranial aneurysms, saccular aneurysms, pulsatile arterial flows, hemodynamics 


\title{
ABSTRACT \\ HEMODYNAMICS OF INTRACRANIAL ANEURYSMS: FACTORS THAT MAY INFLUENCE OCCURRENCE
}

\author{
Francisco A. Pino Romainville
}

The formation and growth of intracranial aneurysms are partly attributed to various hemodynamic factors, such as the wall shear stress and pressure. However, the exact nature of the hemodynamic variables responsible for triggering aneurismal growth is still unknown. The conclusions drawn by various studies in this regard are found to be speculative and sometimes ambiguous. This issue is further investigated in the present work using some new insight gained from passive scalar dispersion in the blood flow for realistic aneurysm geometries. Intracranial aneurysms are best visualized using selective catheter angiogram technique where a contrast agent added to the blood flow is visualized and filmed dynamically. In the current study, the 2D threshold images produced by "3D rotational X-ray angiography technique" are used in an "in-house" program to construct a $3 \mathrm{D}$ volumetric grid which is then used to calculate the blood flow through the aneurysm. The dispersion and flow patterns show the localization of high stress in stagnation areas resulting from secondary flows, which may be the potential regions for the origin and growth of aneurysms. The dependence of flow properties on the initial and boundary conditions to calculate the pressure and the wall shear stress values for a given geometry are investigated. A detailed grid dependence study is also performed to show the dependence of the wall shear stress calculations on the quality of near-wall grid resolution. An estimation of discretization error was made using the procedure recommended by ASME Journal of Fluids Engineering. The results suggest high dependence of calculated WSS on local grid density.

Finally, the aneurysms were virtually removed to identify the hemodynamic factors responsible for aneurysm formation. For these arterial flows without the aneurysm, the results suggest the occurrence of secondary flow impingement patterns and high oscillatory wall shear stress in the regions where the aneurysm was initially present. These factors, over the lifetime of the patient, might lead to the degradation of arterial wall leading to the formation of an aneurysm. 


\section{ACKNOWLEDGMENTS}

I want to thank my advisor and committee chair, Dr. Ismail Celik, not only for giving me the opportunity to work in the bioengineering field, but also more important way he has challenged my way of thinking towards the excellence. All of his wise pieces of advice and patience have accelerated this work thoughtout my $\mathrm{PhD}$ study. I would also like to thank my committee members: Dr. Ansaar Rai, for all of the great discussions that we had in our meetings and his guidance from the medical perspective; Dr. Sam Mukdadi, Dr. David Klinke, and Dr. Wade Huebsch for their good advice, patience, time, and encouragement.

There are some other people I wish to thank for helping me with my dissertation such as Dr. John Kulhman, who advised me about the problems I might encounter with my experiment and offered me his valuable comments; Dr. David Steinman, who provided one of the geometries (aneurysm-A); and Jon Moss, who guided me through the reconstruction of the geometry-B. Finally, to my colleges and friends in the CFD group Francisco and Jose for helping me to run the experiment and their good comments; Jaggu, for his valuable feedback and guidance while working on my dissertation; and the members of the CFD group Ertan, Raju, Nihan, and Don for their helpful observations and great memories that we had during this journey.

I also want to thank to Vanessa Kording for all her love during this time. She has encouraged me every day to work harder and we shared great moments that inspired me to keep going without discouragement. I especially dedicate this work to her, "Mor." She has helped me everyday in this investigation and that is why I want to always be with her. Finally, to my parents and sisters whose love and support had encouraged me through each moment of my life.

This work has been sponsored by West Virginia University Research Corporation 


\section{Table of Contents}

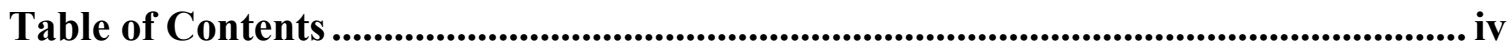

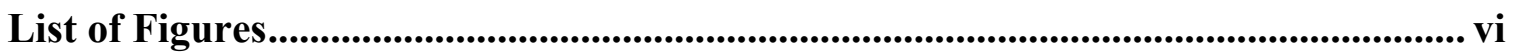

List of Symbols, Abbreviations, and Nomenclature ........................................................ $\mathrm{x}$

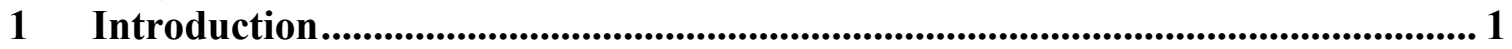

2 Literature Review .......................................................................................................... 6

3 Methodology ……............................................................................................................ 16

3.1 Aneurysms Geometries......................................................................................... 16

3.1.1 Image Acquisition ........................................................................................ 16

3.1.2 Model Reconstruction....................................................................... 17

3.1.3 Mesh Properties .......................................................................................... 20

3.1.4 Fluid and Flow Properties........................................................................... 22

3.1.5 CFD Simulation Parameters..................................................................... 25

3.2 Removal of the Aneurysm ............................................................................. 27

3.2.1 Aneurysm-A geometry with no aneurysm .................................................. 27

3.2.2 Aneurysm-B geometry with no aneurysm .................................................. 28

3.3 Grid Convergence Index (GCI) ........................................................................ 29

4 Results ...................................................................................................................... 31

4.1 Preliminary Study ............................................................................................... 31

4.2 Aneurysms Geometries........................................................................................ 39

4.2.1 Results for Aneurysm-A .............................................................................. 43

4.2.2 Results for Geometry-A without the aneurysm ....................................... 61

4.2.3 Results for Aneurysm-B ............................................................................... 72

4.2.4 Results for Geometry-B without aneurysm............................................ 89

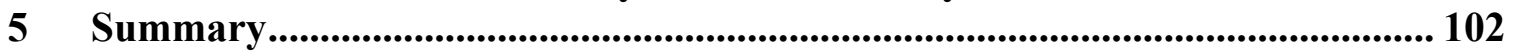

5.1 General Discussion...................................................................................... 109

6 Conclusions and Recommendations................................................................... 112

6.1 Conclusions....................................................................................................... 112

6.2 Recommendations ...................................................................................... 114

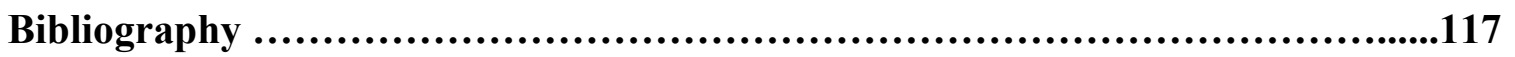

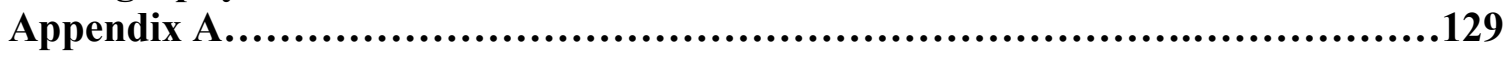

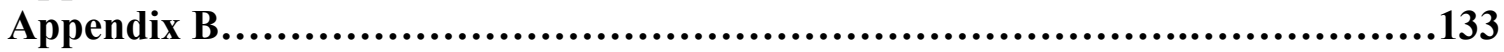




\section{List of Tables}

Table 2.1: Summary of the most relevant literature concerning aneurysms ................ 13

Table 3.1: Summary of the grid resolutions and numerical schemes used for both aneurysms geometries ........................................................................ 21

Table 3.2: Comparative properties between the blood and water ................................. 23

Table 3.3: Summary of the grid resolution for both geometries without the aneurysm ................................................................................ 29

Table 5.1: Summary of all the cases for pseudo-aneurismal and aneurismal

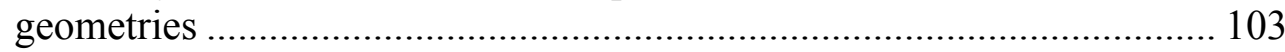

Table A1: Dimensions of the Reconstructed Model Compared to Corcoran's model 130 


\section{List of Figures}

Figure 1.1: Stylized scheme of the blood circulation (Levick, 2003)............................. 2

Figure 1.2: Wall structure of a muscular artery (Levick, 2003) .................................... 3

Figure 2.1: The brain and the arteries of the base of the brain below view (left), Schematic representation of the Circle of Willis (right) (Gray, 1918).......... 6

Figure 2.2: Types of aneurysms: (a) berry (Jozefowicz et al, 2000) or (b) saccular (Rai, private communications), (c) dissecting (Deok et al, 2001), and (d) fusiform (Kang et al, 2005).

Figure 3.1: 3D visualization of Aneurysm-1 shown by the Leonardo software resident to the Siemens X-Ray Angiography Machine; lateral views (top row); aneurysm views (bottom row) (Rai, personal communication)......... 16

Figure 3.2: Iso-surface generation (top row) and removal of small volumes (bottom row) for aneurysm sub-volumes.............................................................. 18

Figure 3.3: (a) Aneurysm-A - anteposterior (top left) and lateral (top right) views; (b) Aneurysm-B xy view (bottom left) and yz view (bottom right)............ 19

Figure 3.4: Inlet flow waveform approximation for one cardiac cycle ( $\mathrm{Ku}$ et al, 1985).

Figure 4.1: WSS comparison and flow axial velocities using a pipe bent with 30 degrees (left) and 60 degrees (right) with respect to the horizontal............ 32

Figure 4.2: WSS components in the $\mathrm{x}$ and $\mathrm{y}$ direction for the bent pipe with 30 degree (left) and with 60 degree (right) with respect to the horizontal....... 33

Figure 4.3: Velocity vectors colored by the dynamic pressure contours $(\mathrm{mm} \mathrm{Hg})$ at different cross sections for a bent pipe with 30 (top) and 60 (bottom) degrees at the systolic peak: Plane-1 (left), Plane-2 (middle), and Plane3 (right)

Figure 4.4: WSS contours of pseudo-aneurysms with 30 (left) and 60 (right) degrees angle bended pipe..................................................................... 36

Figure 4.5: WSS comparison and flow profiles obtained using the coarse grid (left) and the fine grid (right). The axial velocity profiles are compared at the representative lines (dashed lines) on each of the three planes................... 37

Figure 4.6: Velocity profiles comparison and the near wall velocity profiles on three representative planes at the systole peak using the coarse grid (dot line) and the fine grid (dash line): Plane-1 (left), Plane-2 (middle), Plane-3

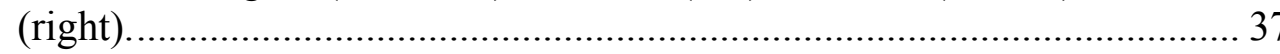

Figure 4.7: Snapshots of flow inside the Aneurysm-A, sagittal plane (left), coronal plane (right) at $\mathrm{t}=1.5,1.65,1.8$, and $2.1 \mathrm{sec}$

Figure 4.8: Snapshots of flow inside the Aneurysm-B, sagittal plane (left), coronal plane (right) at $\mathrm{t}=2.2,2.35,2.5$, and $2.8 \mathrm{sec}$.

Figure 4.9: Location of the cross-sectional planes and a line traced for WSS calculations where results are presented for both geometries with and without the aneurysm (top two images for aneurysm-A and bottom two images for aneurysm-B).

Figure 4.10: Impedance comparison between Pressure Outlet BC and Outflow BC at various locations during a cardiac cycle for Aneurysm-A 
Figure 4.11: Velocity profiles at EA (top) and BF (bottom) cross sections at the systole peak (left) and late diastole (right) for Aneurysm-A using different boundary conditions

Figure 4.12: Velocity profiles at $\mathrm{O} 1$ (top) and $\mathrm{O} 2$ (bottom) cross sections at the systole peak (left) and late diastole (right) for Aneurysm-A using different boundary conditions

Figure 4.13: Scalar concentration in the Aneurysm-A using different boundary conditions at (a) BF cross section at systole (top left) and diastole (top right),(b) $\mathrm{O} 1$ at systole (bottom left), and $\mathrm{O} 2$ at systole (bottom right)...... 48

Figure 4.14: Total pressure at the peak of the systole $(\mathrm{t}=0.25 \mathrm{sec})$ for Aneurysm-A..... 49

Figure 4.15: Velocity (top) and static pressure (bottom) waveform at the geometric center of the inlet cross section (PI) and representative cross-sections EA, $\mathrm{O} 1$ and $\mathrm{O} 2$ during one cardiac cycle for Aneurysm-A

Figure 4.16: Velocity profiles at EA (top) and BF (bottom) cross sections at the systole peak (left) and late diastole (right) for Aneurysm-A using different discretization schemes

Figure 4.17: Velocity profiles at O1 (top) and O2 (bottom) cross sections at the systole peak (left) and late diastole (right) for Aneurysm-A using different discretization schemes

Figure 4.18: Velocity profiles at EA (top) and BF (bottom) cross sections at the systole peak (left) and late diastole (right) for Aneurysm-A using second order discretization scheme.

Figure 4.19: Velocity profiles at O1 (top) and O2 (bottom) cross sections at the systole peak (left) and late diastole (right) for Aneurysm-A using second order discretization scheme.....

Figure 4.20: Wall shear stress contours for Aneurysm-A using different grid resolution at the systole peak: coarse, medium, and fine grid resolution.... 60

Figure 4.21: Results of the WSS along the traced line at the systole peak (left) and oscillatory WSS during a cardiac cycle at three defined points (right) for Aneurysm-A

Figure 4.22: Velocity profiles at different locations of the geometry without the aneurysm at the systole peak (left) and the late diastole (right): BA (top), IS (middle), and AA (bottom)

Figure 4.23: Wall shear stress contours for geometry-A using different grid resolution at the systole peak: coarse, medium, and fine grid resolution.... 65

Figure 4.24: Results of the WSS along the traced line at the systole peak (left) and oscillatory WSS during a cardiac cycle at three defined points (right) for geometry A

Figure 4.25: Velocity vectors colored by the dynamic pressure contours ( $\mathrm{mm} \mathrm{Hg}$ ) for IS cross section with the aneurysm (top) and without the aneurysm (bottom)

Figure 4.26: Velocity vectors colored by the dynamic pressure contours (mm Hg) for AA cross section with the aneurysm (top) and without the aneurysm (bottom)....

Figure 4.27: Impedance comparison between Pressure Outlet BC and Outflow BC at various locations during a cardiac cycle for Aneurysm-B 
Figure 4.28: Velocity profiles at different locations for Aneurysm-B: EA at systole (top left) and diastole (top right), BF at systole (bottom left) and diastole (bottom right) using different boundary condition

Figure 4.29: Velocity profiles at different locations for Aneurysm-B: O1 at systole (top left) and diastole (top right), $\mathrm{O} 2$ at systole (bottom left) and diastole (bottom right) using different boundary condition....

Figure 4.30: Scalar concentration at different locations for Aneurysm-B: (a) BF at systole (top left), BF at diastole (top right), (b) $\mathrm{O} 1$ at diastole (bottom left), and $\mathrm{O} 2$ at diastole (bottom right)....

Figure 4.31: Total pressure (left) and static pressure comparison at cross sections planes immediately before (middle) and after (right) the aneurysm at the systole peak for Aneurysm B

Figure 4.32: Velocity (top) and static pressure (bottom) waveforms at the geometric center of the inlet cross section and representative cross-sections PI, EA, $\mathrm{O} 1$ and $\mathrm{O} 2$ during one cardiac cycle for Aneurysm-B.

Figure 4.33: Velocity Contours at the systole peak $(t=25 \mathrm{sec})$ using different boundary conditions: pressure outlet boundary condition O1 (top left), outflow boundary condition O1 (top right), pressure outlet boundary condition $\mathrm{O} 2$ (bottom left) and outflow boundary condition O2 (bottom right).

Figure 4.34: Static Pressure contours ( $\mathrm{mm}$ of $\mathrm{Hg}$ ) at the outlets: $\mathrm{O} 1$ (left) and $\mathrm{O} 2$ (right).

Figure 4.35: Velocity profiles comparison by using different discretization scheme at the EA cross-section (top) and BF cross-section (bottom) during the systole peak (left) and late diastole (right) for fine grid resolution

Figure 4.36: Velocity profiles comparison by using different discretization scheme at the $\mathrm{O} 1$ cross-section (top) and $\mathrm{O} 2$ cross-section (bottom) during the systole peak (left) and late diastole (right) for fine grid resolution.

Figure 4.37: Velocity profiles comparison by using different grid resolution at the EA cross-section (top) and BF cross-section (bottom) during the systole peak (left) and late diastole (right) for second order discretization scheme

Figure 4.38: Velocity profiles comparison by using different grid resolution at the $\mathrm{O} 1$ cross-section (top) and $\mathrm{O} 2$ cross-section (bottom) during the systole peak (left) and late diastole (right) for second order discretization scheme

Figure 4.39: Wall shear stress contours for Aneurysm-B using different grid resolution at the systole peak: coarse, medium, and fine grid resolution.... 88

Figure 4.40: Results of the WSS along the traced line at the systole peak (left) and oscillatory WSS during a cardiac cycle at three defined points (right) for Aneurysm-B

Figure 4.41: Velocity profiles comparison using different grid resolution during the systole peak (left) and late diastole (right) for BA, IS, and AA cross sections

Figure 4.42: Contours of WSS at the systole peak for three different grid resolutions: coarse (left), medium (middle), and fine (right) grid resolutions. 
Figure 4.43: Results of the WSS along the traced line at the systole peak (left) and oscillatory WSS during a cardiac cycle at three defined points (right) for geometry-B

Figure 4.44: Velocity vectors colored by the dynamic pressure contours (mm Hg) for BA cross section with the aneurysm (top) and without the aneurysm (bottom)

Figure 4.45: Velocity vectors colored by the dynamic pressure contours ( $\mathrm{mm} \mathrm{Hg}$ ) for IS cross section with the aneurysm (top) and without the aneurysm (bottom)

Figure 4.46: Velocity vectors colored by the dynamic pressure contours (mm Hg) for AA cross section with the aneurysm (top) and without the aneurysm (bottom)

Figure 5.1: Bovine endothelial cell exposed to physiologic shear stress $>1.5 \mathrm{~N} / \mathrm{m}^{2}$, (left) and $\pm 0-4 \mathrm{~N} / \mathrm{m}^{2}$ (right) after 24 hours (Malek et al, 1999).

Figure 6.1: Specific-patient volumetric waveform for a cardiac cycle (Plesniak et al, 2007).

Figure A1: Geometry reconstruction comparison between Corcoran's procedure and our procedure

Figure A2: Upper respiratory track geometry imported in Gambit.....

Figure A3: Velocity vectors colored by the $\mathrm{z}$ velocity in the upper respiratory track during the coughing peak (left) and coughing decelerating phase (right)

Figure B1: Y-pipe close-up used in the experimental set-up.....................133

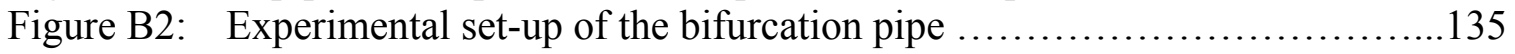

Figure B3: Geometrical representation of the Y-pipe used in the simulation...........136

Figure B4: Volumetric flow rate comparison between the experiments and the numerical simulations

Figure B5: Qualitative comparison results of the fluid flow characteristics for the bifurcation model: Velocity vectors colored by the axial velocity at $\mathrm{Re}=1,200$ (right) and $\mathrm{Re}=1,100$ (left) (Zeller et al, 1970).

Figure B6: WSS contours of the bifurcation model: $\mathrm{Re}=1,700$ (left) and $\mathrm{Re}=1,200$ (right)

Figure B7: Pulse form and flow fluctuation (Zeller et al, 1970) 140 


\section{List of Symbols, Abbreviations, and Nomenclature}

$\begin{array}{ll}\text { Abbreviations: } \\ \mathrm{d} & \text { Diffusivity } \\ \mathrm{f} & \text { Friction factor } \\ \mathrm{m} & \text { Mass flow rate } \\ \mathrm{n} & \text { Representative cell size } \\ \mathrm{p} & \text { Apparent order of the method } \\ \mathrm{r} & \text { Grid refinement factor } \\ \mathrm{s} & \text { Artery direction } \\ \mathrm{t} & \text { Time } \\ u_{s} & \text { Velocity along the s-direction } \\ \mathrm{u}, \mathrm{v}, \mathrm{w} & \text { Cartesian velocity components } \\ \mathrm{A} & \text { Cross sectional area } \\ \mathrm{C}_{\mathrm{f}} & \text { Friction coefficient } \\ \mathrm{D} & \text { Diffusion } \\ \mathrm{K}_{\mathrm{B}} & \text { Boltzmann constant } \\ \mathrm{N} & \text { Total number of cells } \\ \mathrm{P} & \text { Pressure } \\ \mathrm{Q} & \text { Volumetric flow rate } \\ \mathrm{R}_{0} & \text { Molar radius of the solute } \\ \mathrm{Re} & \text { Reynolds number } \\ \mathrm{T} & \text { Temperature } \\ \mathrm{V}_{\mathrm{i}} & \text { Volume of the computational cell } \\ \mathrm{Z} & \text { Characteristic Impedance } \\ & \end{array}$

Greek letters:

$\begin{array}{ll}\rho & \text { Density } \\ \Gamma & \text { Diffusivity } \\ \mu & \text { Dynamic viscosity } \\ \varsigma_{P} & \text { Perimeter of the artery } \\ \Phi & \text { Point on a profile for a grid resolution } \\ \tau_{w} & \text { Wall shear stress }\end{array}$


Acronyms:

$\begin{array}{ll}\text { AA } & \text { After the aneurysm } \\ \text { BA } & \text { Before the aneurysm } \\ \text { BC } & \text { Boundary conditions } \\ \text { BF } & \text { Before the bifurcation } \\ \text { EA } & \text { Entrance of the aneurysm } \\ \text { FEA } & \text { Finite element analysis } \\ \text { FSI } & \text { Fluid-Solid Interaction } \\ \text { GCI } & \text { Grid Convergence Index } \\ \text { IS } & \text { In situ where the aneurysm was placed } \\ \text { O1 } & \text { Large artery outlet } \\ \text { O2 } & \text { Small artery outlet } \\ \text { PI } & \text { Parental or primary inlet } \\ \text { RE } & \text { Richardson extrapolation } \\ \text { UDS } & \text { User Define Scalar } \\ \text { WSS } & \text { Wall shear stress }\end{array}$




\section{Introduction}

In order to understand cerebrovascular diseases, it is important first to know the cardiovascular and cerebrovascular physiology fundamentals. Among the cardiovascular physiology, the blood is irrigated throughout the human body through arteries and it returns through veins. The veins and arteries are interconnected by capillaries. The organ in charge of this pumping process is the heart. This muscle is divided in four cavities: left and right ventricles and atriums. Deoxygenated blood is pumped from the right ventricle to the lungs through the pulmonary artery and oxygenated blood returns to the left atrium. This short circulation is known as pulmonary circulation. Equal volume of oxygenated blood is pumped from the left ventricle to the rest of the body and deoxygenated blood returns to the heart through two main veins (vena cava superior and inferior) to the right atrium. This complete cycle is known as systematic circulation (Harvey W., 1628). Figure 1.1 shows a stylized scheme of the blood circulation. The circulation processes occur during repetitive cardiac cycles in which the blood travels as explained above. A cardiac cycle mainly consist in two phases: the ventricles receive blood while the muscle is relaxed (diastole) and when the blood is expelled from the atriums, and consequently the muscle is contracted (systole).

The volume ejected per contraction in a healthy adult is $70-80 \mathrm{~mL}$ per min and the heart rate is $60-75$ beats/min (5 liters per min) (Levick, J. R., 2003). However, the cardiac output is variable because it adapts to changing internal or external circumstances such as exercise, nerves, etc. Any change of the blood circulation is immediately detected by the brain tissue because this consumes about the $20 \%$ of the total quantity of the oxygen 
utilized by all tissues in the body (Mchedlishvili, 1986). The brain tissue is the main source of energy consumed (oxidation of carbohydrates) and it exhibits a high sensibility of any blood disturbance. Therefore, the brain tissue is highly vulnerable to pathogenic factors mostly related to circulatory disorders. For example, if the blood flow is completely stopped, it would cause the death of the brain tissues after 4-5 minutes (Scheinberg and Joyne, 1952). Hence, the brain is a highly vulnerable organ for any change in the blood circulation and it has the priority in blood supply over the other organs (Mchedlishvili, 1986).

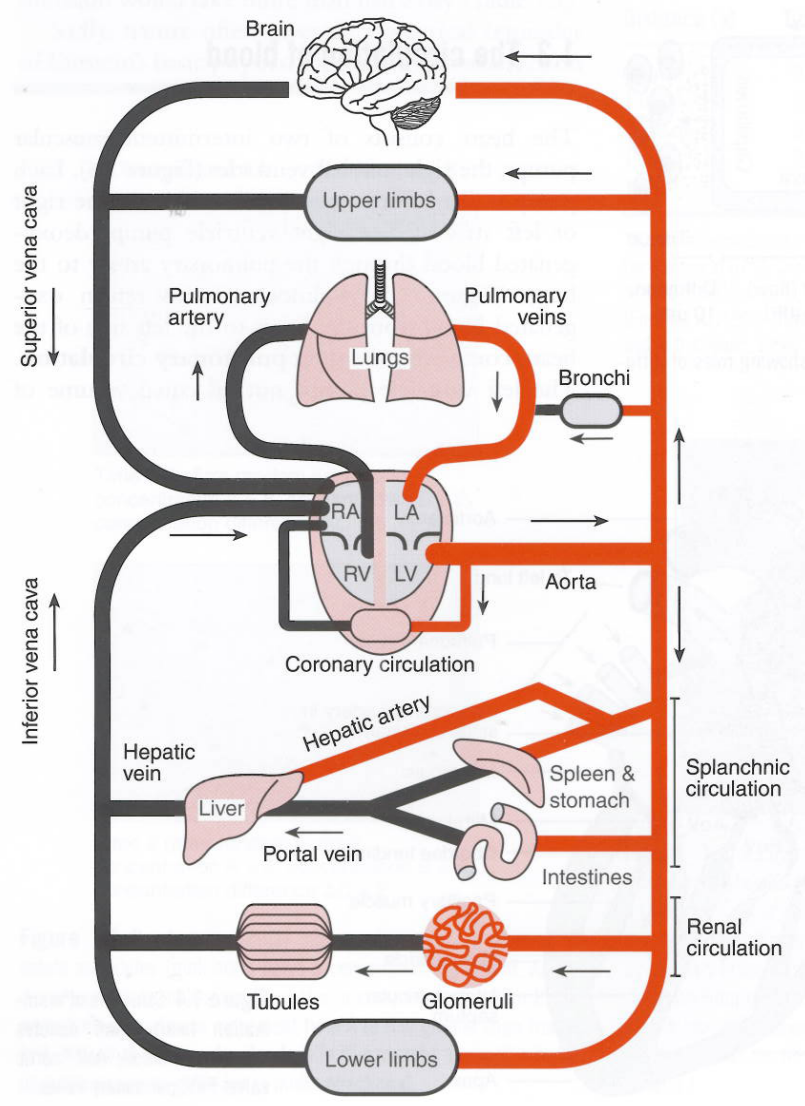

Figure 1.1: Stylized scheme of the blood circulation (Levick, 2003)

The walls of all arteries (except capillaries) are made up of three layers: the tunica intima (the innermost coat or endothelium), tunica media (the middle coat that consists on 
vascular smooth muscle, collagen, and elastin), and tunica adventitia (the outer coat or connective tissue). The first layer or intima is a sheet of flattened endothelial cells. The main function of this layer is to avoid the escape of plasma. The media or second layer supplies mechanical strength and contractile power through the elastin and collagen fibres. Elastin is an extracellular protein which enables the artery to expand by $\sim 10 \%$ during each heart beat, while collagen is also an extracellular protein which forms a strong fibrils network in the media. Finally, the last layer, adventitia, maintains the vessel loosely to the surrounding tissue. All three layers are interconnected by an elastic lamina: internal (between the intima and media) and external (between the media and adventitia). Figure 1.2 shows a detailed wall structure of muscular artery (Levick, 2003).

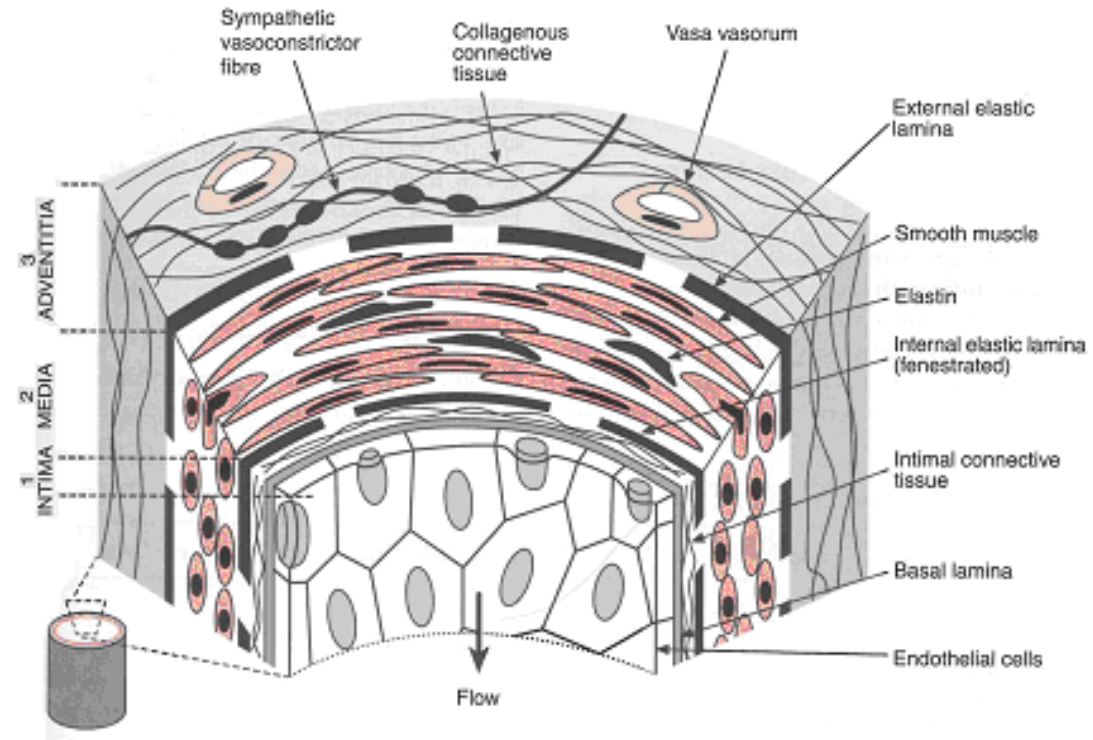

Figure 1.2: Wall structure of a muscular artery (Levick, 2003)

According to polls, cerebrovascular diseases are the third leading causes of death in the United States in 2004 (Miniño et al., 2006), caused generally by high blood pressure or diabetes. Intracranial aneurysms are categorized as one of the artery-vein brain diseases which tend to occur at the main aorta and at the base of the brain (circle of 
Willis). Although many hypotheses exist presently related with this topic, it is still unclear what origins such disease. For example, in previous studies, it is found that high wall shear stress plays an important hemodynamic role in the formation and development of aneurysms. However, some other researchers such as Shojima et al, (2004), assert that very low shear stress is the main factor that indicates structural fragility on the aneurismal walls. This information is contradictory and can mislead future researchers in the neuroscience field.

In the past, many hemodynamic studies about aneurysms were used in non-real geometries (pseudo-geometries), while few studies used real aneurysms geometries. This was largely due to limited visualization equipment. However, with the use of powerful computers and advanced image processing techniques, the use of real geometry is more feasible. These complex geometries can be reconstructed from 2D images obtained from computer tomography (CT) or magnetic resonance imaging (MRI) using commercial or custom own developed codes. The disadvantage of the commercial codes is the high price in the market, and also the time it takes to learn how to use the software, among the most important reasons. On the other hand, a custom code with advanced image processing package developed by the user yield the reconstruction of the geometry with only the time as unique disadvantage.

In order to proceed with this investigation, a preliminary study is performed on a pipe flow with a similar diameter as one of the aneurismal geometries. Although this pseudo-geometry would only elucidate some of the hemodynamic factors that may trigger the aneurismal growth, this is the first approach to understand the flow characteristics in a curved pipe without any other geometrical features (variable diameter, 
bifurcation, etc.). In order to emulate the cardiac cycle, a pulsatile flow similar as past investigations is imposed at the inlet with a periodic duration of 0.7 seconds. The study of the bend is investigated with and without a bulge. Consequently, the two real geometries are studied using the same cardiac cycle with and without the aneurysm. As mentioned before, these geometries are reconstructed by using this image processing technique developed in user-friendly software.

The blood flow can be simulated by using an appropriate CFD (Computational Fluid Dynamics) code and the results elucidate which parameters affect the aneurysm growth in real geometries. The geometry is also modeled by removing the aneurysm virtually in order to determine what parameters may contribute to the origin of intracranial aneurysms. Several cross sections are made on the region of interest (where the aneurysm is placed) to find out such parameters. Hence, the reasons as to why the aneurysms occur (origin and development), which are currently not well understood, will be clarified in the present investigation. These results may help physicians have a better understanding of not only how to treat the aneurysm, assuring adequate handling, and reducing the probability of reoccurrence, but also in developing devices for better treatment. 


\section{Literature Review}

Intracranial aneurysms are pathological dilatations that tend to occur at or near to the arterial bifurcation, mainly in the circle of Willis (Steinman et al, 2003; Isoda et al, 2006; and Stehbens, 1989) (Figure 2.1) region of the brain. Aneurysms develop because of the wall weakness in the vessels and have a tendency to the rupture if not treated in time. Cerebral aneurysms can be grouped in the following categories: berry or saccular, dissecting, and fusiform (Figure 2.2).

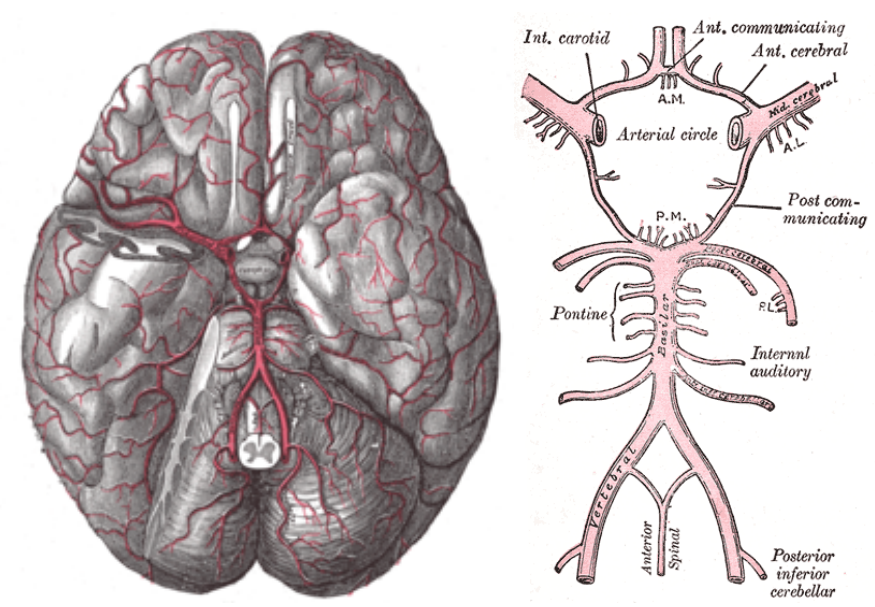

Figure 2.1: The brain and the arteries of the base of the brain below view (left), Schematic representation of the Circle of Willis (right) (Gray, 1918)

The most common aneurysm is the berry or saccular aneurysm, which is named differently depending on the vessel direction and the aneurysm position. The vessel is usually curved and the aneurysm occurs on a side as shown in Figure 2.2b. Simplified ideal models have a straight parental vessel maintaining the aneurysm on a side. Additionally, aneurysms can grow at a bifurcation of two vessels. Aneurysms can occur 
at a symmetric bifurcation (basilar or middle cerebral artery bifurcation) or can form at an angle with the plane of bifurcation (in which case it is called bleb).

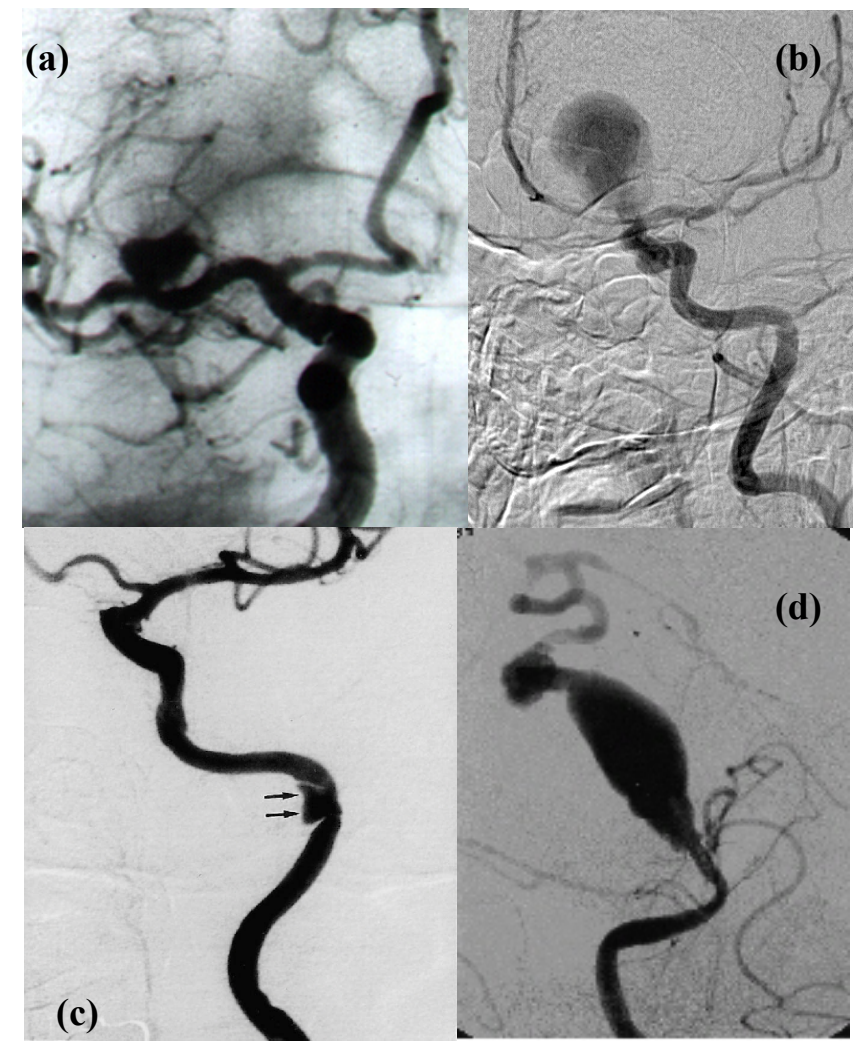

Figure 2.2: Types of aneurysms: (a) berry (Jozefowicz et al, 2000) or (b) saccular (Rai, private communications), (c) dissecting (Deok et al, 2001), and (d) fusiform (Kang et al, 2005)

It is estimated that about 5 million people in the US exhibit cerebral aneurysms according to Kassell and Torner poll studies (Kassell and Torner, 1982). Some previous investigations (Utter et al, 2006; Steinman et al, 2003; Mantha et al, 2006; Feng et al, 2004; Jou et al, 2003; Foutrakis et al, 1999; and Gonzales et al, 1992) have attributed the origin and formation of brain aneurysms to various hemodynamic factors such as shear stress, pressure, and stagnation regions. Hemodynamic stress has a profound effect on the development of cerebral aneurysms (Shojima et al, 2004; Stehbens et al, 1989; Steiger et al, 1990). Not only is the growth of the aneurysm observed near the zones of maximum 
wall shear stress, the growth could also be related to the prolonged residence times of suspended blood cells that may affect the structure of the aneurysm wall (Jou et al, 2005). In addition, Mantha's investigation had shown that temporary reversal of WSS could trigger pathologic changes in the endothelial cells, leading to aneurysms formation (Mantha et al, 2006). Similarly, eventual aneurysms formation sites can be influenced by strong contributory secondary flows. These secondary flows may occur due to the obstruction in some regions of the arteries (cholesterol accumulated on the walls). Mantha's investigation also showed that the highest WSS occurred near the bends when the aneurysm was removed and the arterial geometry was reconstructed. In some other studies (Steinman et al, 2003; Feng et al, 2004; Jou et al, 2003; Shojima et al, 2004), in which the aneurysm was not removed, the WSS was high around the neck of the aneurysm. Finally, some researchers have modeled the growth of aneurysms in simple geometries (pseudo-aneurysms) (Feng et al, 2004, and Foutrakis et al, 1999).

Due to the complexity of the arterial geometry, ideal geometries were initially modeled to represent real geometries. However with the development of new sophisticated techniques such as computed tomography angiography (CTA) and magnetic resonance angiography (MRA), it is possible to visualize the exact aneurysm geometry. These types of visualization are possible through the injection of the contrast agent in the arterial and venous supply and capturing arterial geometry as the contrast agent flows through.

The shape of the aneurysm and the parental vessel is commonly reconstructed from 2D images and is the most important step towards complete analysis because of its strong influence on the hemodynamics (Utter et al, 2006; Steinman et al, 2003; and 
Shojima et al, 2004). These CTA or MRI procedures include not only the imaging data, but also the monitoring of the patients vital signs. The requirement of the accurate physiologic flow conditions for each individual patient is also important to have a better approximation of the degeneration of the vessels (Cebral et al, 2005). The blood flow waveforms associated with each patient may affect the hemodynamic stresses and pressures. However, it was demonstrated that by changing the magnitude of the blood waveform, there is no major changes in the flow pattern. The maximum and minima in WSS was found to occur at the same locations when the waveform varied (Mantha et al, 2006). However, in strict fluid dynamical sense, WSS changes arise from changes in the fluid flow, however small they may be. Similarly, experimental studies (Ford et al, 2003), had shown that gravity has very little effect on the contrast agent during the performance of the angiography.

Feng et al (2004), conducted a numerical simulation of the growth of a pseudosidewall-aneurysm, in which he concluded that the genesis of the aneurysms is due to high WSS concentrated near the distal neck region. As the aneurysm starts enlarging, the neck region becomes part of the aneurysm sac. The aneurysm keeps growing until it is treated or rupture occurs. Results show that during the development of the aneurysms, high WSS is observed at the distal and proximal end of the aneurysms. This is due to the interaction between the WSS and the degeneration of the wall. During the enlargement phase, fluctuations of WSS are present mostly in the proximal neck region (Feng et al, 2004). The enlargement of impingement zones can occur if the arterial curvature is amplified (Hoi et al, 2004). On the other hand, bleb aneurysms developed differently in relation to the sidewall aneurysm. During the cardiac cycle, the bifurcation of the arteries 
is in the primary location of high hemodynamic stress because the blood impinges on the wall. Due to the dilatation process of the distal neck caused by high pressure and shear rates, a saccular aneurysm develops. Further growth may occur in the neck and not the dome of the aneurysms. A narrow-mouthed neck has a higher propensity for thrombus formation when compared to the wide-mouthed neck (Foutrakis et al, 1999). Other investigations related to saccular aneurysms have concluded that high-speed flow entering the aneurysm leads to persistent and transient vortices. These vortices occur at the proximal and distal ends of the neck, producing dynamic patterns of elevated and oscillatory wall shear stresses (Steinman et al, 2003). The position and magnitude of these vortices is due to pulsatile flow conditions rather than the wall's elasticity. However, the wall maintains the needed pressure gradient for the vortex development (Miller et al, 2005). Numerical simulations (Miller et al, 2005, and Chintavis et al, 1995) also show a stronger and more clearly developed vortex close to the aneurysm neck.

Funamoto et al (2007), had conducted experiments using ultrasound probes to record the boundary conditions for numerical simulations. The experimental study shows a relatively large error compared to the numerical investigation due to the recirculation near to the walls. Also, the velocity magnitude showed a large error due to the upstream velocity profile which becomes different at the deceleration phase when compared to the simulations. Therefore, Funamoto proposes a model with feedback at the boundary conditions to decrease the error during the numerical simulation. Gonzales et al (1992), also observed in this pseudo-aneurysm model a major change in the blood flow. A vortex flow with a clockwise rotation was found in the acceleration period of the systole and a counterclockwise rotation during the deceleration period. Therefore, these oscillatory 
WSS would cause wall lesions and as a result would trigger the formation of the aneurysm in this region.

Most of the previous investigations have made rigid wall assumptions in modeling the visco-elastic arterial walls. In this regard, some studies (Shojima et al, 2004), were carried out with moving walls showing that the WSS magnitude is overestimated compared to the results using rigid walls. However, other viscoelastic wall simulations (Dempere-Marco et al, 2006) had shown consistent estimations of the local flow and size of the flow impact zone with the rigid wall assumption.

Turbulent flow can occur at the systole peak during the pulsatile arterial flow. Transitional flow is currently very difficult to model (Younis et al, 2004). In order to simulate pulsatile turbulent flow, a successful model requires: (a) correct capture of the non-equilibrium effects due to the interactions of the mean-flow unsteadiness with the random turbulence, (b) correct reproduction of the effects of the laminar-turbulent transitional behavior that occur at the cardiac cycles, and (c) good predictions of the nearwall flow. Younis et al (2004) claimed that the results obtained are not very promising because experimental data of the WSS differ from the simulations by $40 \%$ approximately. However, the model q- $\zeta$ achieved the best results compared to the others for a simple eddy-viscosity turbulence closure.

The aneurysm walls seem to have less elastic behavior (two times less than a health vessels approximately) and are stiffer than the surrounding tissues (Toth et al, 2003). Thus, the magnitude of WSS in the aneurysm region is much lower than the parent vessels (Shojima et al, 2004). Some CFD studies (Jou et al, 2003; Perktold and Rappitsch, 1995) have shown that the wall motion has a relatively small effect on the 
flow in large arteries where the shear strain rate is small. On the other hand, aneurysm cases with a bleb are highly affected by the wall motion (Dampere-Marco et al, 2006; Isida et al, 2005; Hayakawa et al, 2005).

Complex surgical interventions are required to treat intracranial aneurysms. The traditional method used from the 1980s to the 1990s is known as clipping where a craniotomy is performed. It is a surgical operation in which a part of the skull is cut in order to access the brain. However, in the late 1990s a new method emerged, named coil embolization. This consists of filling in the aneurysm with coils through a catheter. Coils can relieve the influx of pulsating blood, allowing the initial clotting and thus eliminating the aneurysm. This effect is usually obtained when a volume density of $20 \%$ platinum coil is injected inside the aneurysms (Gorden et al, 2001). The success rate of coil embolization is believed to be about $70-80 \%$. In other words, the symptoms reoccur in a relatively high percentage (33.6\%) of treated patients (Raymond et al, 2003), and subsequently bleeding inside the aneurysm occurs. On the other hand, not every aneurysm can be treated with the same method. Usually, wide-necked aneurysms are treated with stents, an expandable dispositive that counteracts a flow constriction in a reduced or blocked vessel. It can also be used to reduce or shift the vessel curvature in order to decrease the inflow momentum, WSS, and the impact zone size to the distal zone. Moreover, these devices can slow down or stop the aneurysms growth, preventing the risk of rupture in the aneurysms (Meng et al, 2006; Canton et al, 2005). 
Table 2.1: $\quad$ Summary of the most relevant literature concerning aneurysms

\begin{tabular}{|c|c|c|c|c|c|c|c|c|c|}
\hline Author & Aneurysm & $\begin{array}{l}\text { Velocity } \\
\text { Field }\end{array}$ & Fluid & Walls & Dim & Type & Treat & Other Comments & WSS \\
\hline $\begin{array}{l}\text { Steinman et al } \\
(2003)\end{array}$ & real case $(\mathrm{g})$ & pulsatile & $\begin{array}{l}\text { Newtonian \& } \\
\text { non-Newtonian fluid }\end{array}$ & Rigid & $3 \mathrm{D}$ & CFD & none & qualitative validation & coximal and \\
\hline Mantha et al (2006) & 3 real cases & pulsatile & $\begin{array}{l}\text { incompressible blood flow } \\
\text { as Newtonian fluid }\end{array}$ & Rigid & $3 \mathrm{D}$ & CFD & none & $\begin{array}{l}\text { study with and without } \\
\text { aneurysm }\end{array}$ & $\begin{array}{l}\text { Small WSS locat } \\
\text { aneurysms was virtu }\end{array}$ \\
\hline & & & (1) & & & & coil & (1) & round the neck of \\
\hline Dem & o tea & puisatile & JNen & Igid & & & & & \\
\hline al $(2006)$ & 3 real cases & pulsatile & New & moving & $3 \mathrm{D}$ & CFD & none & & \\
\hline $\begin{array}{l}\text { Shojima et al } \\
(2004)\end{array}$ & & & $\begin{array}{l}\text { incompressible blood flow } \\
\text { as Newtonian fluid }\end{array}$ & Rigid & & & & & \\
\hline Ford et al (2003) & pseudo model & $\begin{array}{l}\text { Pulsatile }+ \\
\text { steady }\end{array}$ & glycerol+water & Rigid & $3 \mathrm{D}$ & Exp & none & $\begin{array}{l}\text { effects of } \\
\text { angiograph }\end{array}$ & \\
\hline Jou & a real & pulsatile & New & Rigid & $3 \mathrm{D}$ & CFD & none & basilar aneurysm & Low WSS b \\
\hline $\begin{array}{l}\text { Foutrakis et al } \\
(1999) \\
\text { Bluestein et al } \\
(1996)\end{array}$ & $\begin{array}{l}2 \text { pseudo } \\
\text { models } \\
\text { pseudo model }\end{array}$ & pulsatile & $\begin{array}{l}\text { incompressible blood flow } \\
\text { as Newtonian fluid } \\
\text { blood as Newtonian fluid }\end{array}$ & $\begin{array}{l}\text { Rigid } \\
\text { Rigid }\end{array}$ & $3 \mathrm{D}$ & $\begin{array}{l}\text { CFD } \\
\text { Exp+ } \\
\text { CFD }\end{array}$ & none & $\begin{array}{l}\text { leb aneurysm } \\
\text { turbulent } \\
\text { del) }\end{array}$ & $\begin{array}{l}\text { High WSS at the neck of the bleb } \\
\text { during the genesis and growth } \\
\text { Turbulent WSS one order of } \\
\text { magnitude higher than laminar }\end{array}$ \\
\hline Isoda et al (2006) & & pulsatile & $\mathrm{n} / \mathrm{a}$ & Rigid & $3 \mathrm{D}$ & $\operatorname{Exp}$ & none & & None \\
\hline Meng et al (2006) & $\begin{array}{l}2 \\
\text { models }\end{array}$ & steady & glycerin+water & Rigid & $3 \mathrm{D}$ & $\begin{array}{l}\text { Exp }+ \\
\text { CFD }\end{array}$ & stent & $\begin{array}{l}\text { Study } \\
\text { sided }\end{array}$ & Stent reduces the WS \\
\hline Utter et al (2006) & pseudo model & 1 pulsatile & blood as Newtonian fluid & Rigid & $3 \mathrm{D}$ & CFD & none & $\begin{array}{l}\text { the flow } \\
\text { al forces. } \\
\text { atinum }\end{array}$ & $\begin{array}{l}\text { Low WSS promote development } \\
\text { and trigger the rupture } \\
\text { None }\end{array}$ \\
\hline Groden et al (2001) & pseudo model & 1 pulsatile & blood as Newtonian fluid & Rigid & $3 \mathrm{D}$ & CFD & coil & & \\
\hline Canton et al (2005) & pseudo model & 1 pulsatile & glyc & Rigid & $3 \mathrm{D}$ & Exp & stent & differe & iple stent \\
\hline Mil & 1 & & glyce & gid & & $\begin{array}{l}\text { Exp }+ \\
\text { CFD }\end{array}$ & & Vortex position as function & High WSS at \\
\hline $\begin{array}{l}\text { Acevedo-Bolton et } \\
\text { al (2006) }\end{array}$ & real case $(g)$ & $\begin{array}{l}\text { Pulsatile + } \\
\text { steady }\end{array}$ & & Rigid & $3 \mathrm{D}$ & $\begin{array}{l}\text { Exp }+ \\
\text { CFD }\end{array}$ & none & $\begin{array}{l}\text { growth of aneurysm } \\
\text { analyzed once a year }\end{array}$ & $\begin{array}{l}\text { Low WSS related to prolonged } \\
\text { residence times degenerate wall }\end{array}$ \\
\hline Feng & pseudo model & steady & $\begin{array}{l}\text { incompressible Newtoni } \\
\text { fluid }\end{array}$ & Rigid & $3 \mathrm{D}$ & & none & growth of aneurysm & $\begin{array}{l}\text { Development of aneurysm } \\
\text { sensitive to the high WSS }\end{array}$ \\
\hline $\begin{array}{l}\text { Tittelbaugh et al } \\
\text { (2007) }\end{array}$ & $T^{2}$ & & $\begin{array}{l}\text { blood } \\
\text { non-Newtonian fluid }\end{array}$ & & & $\begin{array}{l}\text { Exp+ } \\
\text { CFD }\end{array}$ & & 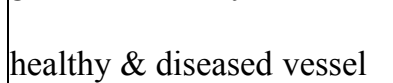 & \\
\hline
\end{tabular}




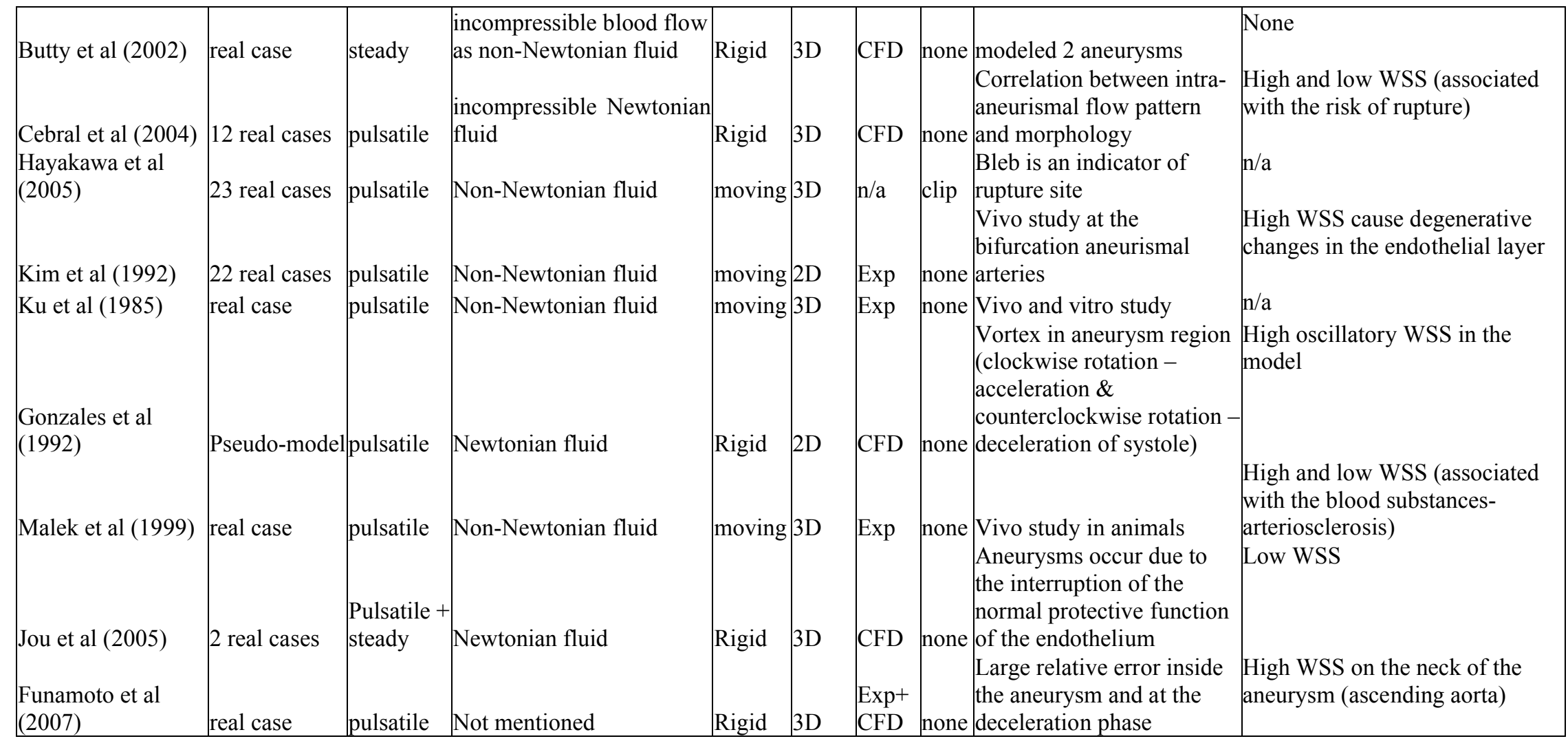


As shown in the above literature review [see Table 2.1], there has been an extensive research on the subject of aneurysm formation, growth, and rupture. However, reasons and the mechanisms of each of these stages are still not clearly understood. As a consequence, the exact hemodynamic factors that play a decisive role in the origin and growth of intracranial aneurysms are still unknown. In the past, extensive investigations had been made using pseudo-aneurysms geometries to find possible answers such as the WSS and stagnation regions. Although some conclusions had been drawn from these approximations, real geometries are more complex due to the irregular shape of the lumen vessels. Presently, it is more feasible to carry out these investigations by the use of powerful computers which facilitate the study. Such studies can be made from the reconstruction of the geometries to blood flow simulation in aneurismal geometries. Cerebral blood flow simulations using real geometries have been carried out for the last 10 years finding, in many cases, contradictory results. These results have shown the WSS as the most controversial factor in cerebral aneurysms. There are two main tendencies that researchers claim to cause this disease: the high and low WSS. Many of studies speculated on the nature of the WSS on a single grid resolution in CFD simulations when the WSS had a strong dependence on the resolved velocity gradient near to the wall, which in turn depended on the near wall mesh. Therefore, the goal of the present investigation is to clarify which hemodynamic factors might lead to occurrence of cerebral aneurysms. The present study would focus in the fluid flow characteristics (mainly velocity and WSS) with no artery wall interaction, through three different grid resolutions, although it is known that the internal wall structure will change due to the shear of the blood flow. 


\section{Methodology}

\subsection{Aneurysms Geometries}

\subsubsection{Image Acquisition}

An X-ray angiogram was performed (Rai, 2006) on a 66-year-old female who presented a giant intracranial aneurysm. Using a rotational arm constructed by Siemens, the images were obtained and recorded in four sets of 100 pictures each. The 2D monochromatic images were obtained using the Leonardo software which also computes a 3D visualization of the aneurysm. A nonionic diametric contrast medium (Visipaque Iodixanol $320 \mathrm{mgI} / \mathrm{mL}$ ) was injected during angiography to underline the artery boundaries along the vessel and to record lumen of the arteries as shown in see Figure 3.1 .

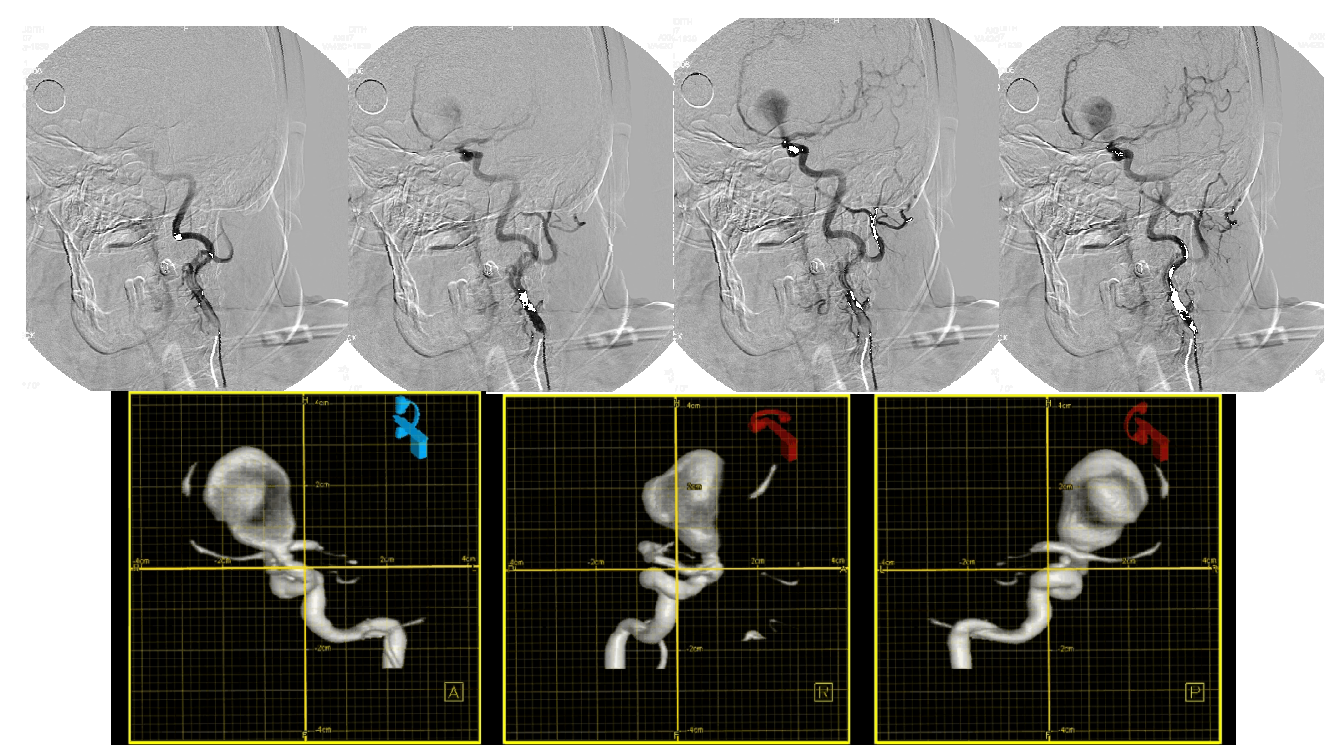

Figure 3.1: 3D visualization of Aneurysm-1 shown by the Leonardo software resident to the Siemens X-Ray Angiography Machine; lateral views (top row); aneurysm views (bottom row) (Rai, personal communication) 


\subsubsection{Model Reconstruction}

The rotational angiogram produced images of resolution $512 \times 512$ pixels, and the slice distance (pitch) among each image was $0.213158 \mathrm{~mm}$. The methodology (Nanduri et al, 2007) to perform the reconstruction of the geometry is divided into three steps: image acquisition, geometry extraction (iso-surfaces), and grid generation. These images were processed through MATLAB (Mathworks Inc., Natick, MA) to capture the vessel boundaries and generate an iso-surface based on the threshold of the image. Image processing functions are available in the MATLAB image processing toolbox. The threshold information was read for each consecutive slice generating a 'voxel' matrix. By using the MATLAB 'graythresh' function, the global threshold was calculated on each slide to be transformed in a binary image. A trial and error sequence was adopted until the artery cross sectional area of the reconstructed geometry approximately matched with the ones obtained by the Leonardo software. These comparison processes of cross sectional areas are chosen randomly. During this process, some spurious 'islands' are found inside the aneurysm due to concentration variation of the fluid contrast (brightness). Swirling flows inside the aneurysm may be the reason for such variation on the brightness in contrast agent. The total volume of the geometry is not suitable to be reconstructed in only one piece due to the memory requirements and the size of the total domain, so it is divided into sub-volumes to be joined later. The binary filter is applied using the same threshold value to match the boundary regions between the sub-volumes. This binary matrix can be generated by using the 'isonormal' and 'isosurface' MATLAB functions. During the reconstruction process, the outlying arteries can be found and they are eliminated leaving the three arteries: parental artery and two child-arteries (see Figure 
3.2). The 'bwareopen' MATLAB function removes all the small objects from the main volume (region of interest). Each sub-volume was exported in a STL (Standard Tessellation Language) format which is the most appropriate to be read in grid generation software. Inlets and outlet(s) cross-sections were cut to include the "twist and bend" in the artery leading to the aneurysm and the two bifurcation branches after the aneurysm. In order to make the outlet cross-sections perpendicular to the flow direction, 3Matic software (Materialise, Leuven, Belgium) was used.
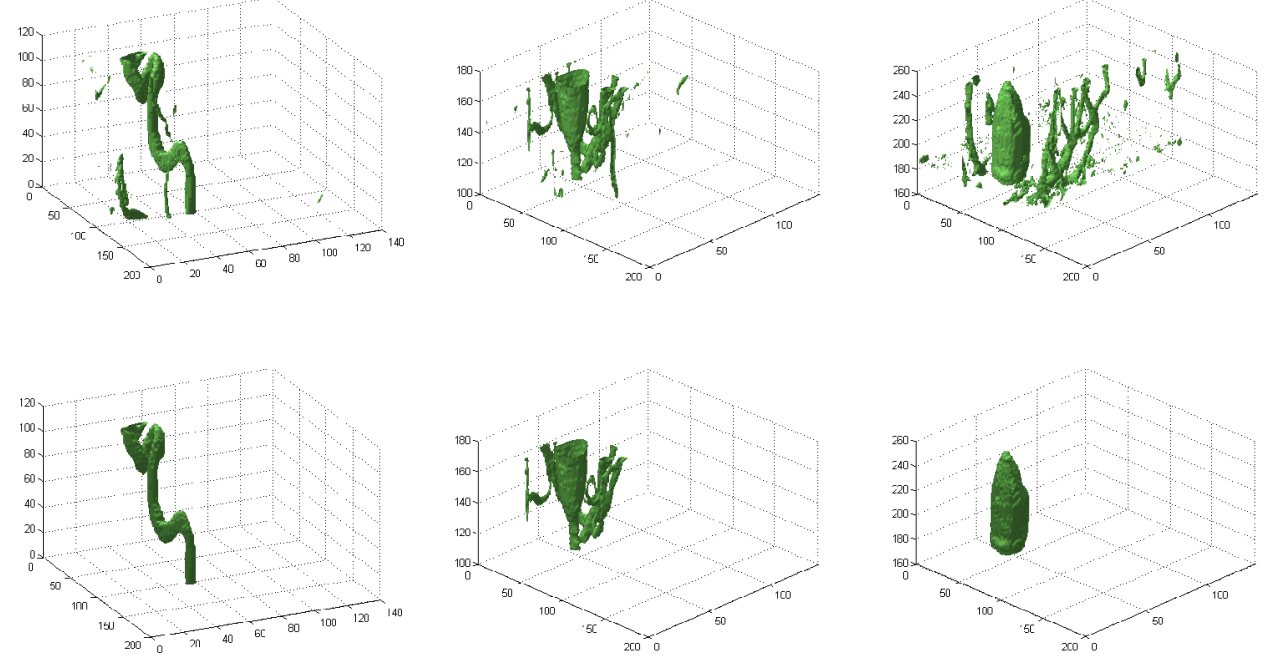

Figure 3.2: Iso-surface generation (top row) and removal of small volumes (bottom row) for aneurysm sub-volumes

As a qualitative comparison, a giant carotid-posterior communicating artery aneurysm was reconstructed using the point cloud and the connectivity matrix provided by Dr. Steinman et al. (2003) of Robarts Research Laboratory, Ontario, Canada. This point cloud and the connectivity matrix were generated from the angiogram images using methods tabulated in Steinman et al, 2003. Figure 3.3a shows the 3D geometry of this aneurysm (henceforth called Aneurysm-A). The bottom half of Figure 3.3b shows the 3D 
geometry of this aneurysm (henceforth called Aneurysm-B) with the inlet and the outlet cross-sections.

The reconstruction of the aneurysm-B geometry is validated in the Appendix A using the 'in-house' software. Another way to validate this reconstruction is to use a silicon model. However, due to the high price of this device, the first method was used to corroborate such reconstruction. Applying the same MATLAB code that was used to reconstruct the Aneurysm-B, it was used to rebuild a validated upper respiratory track geometry.
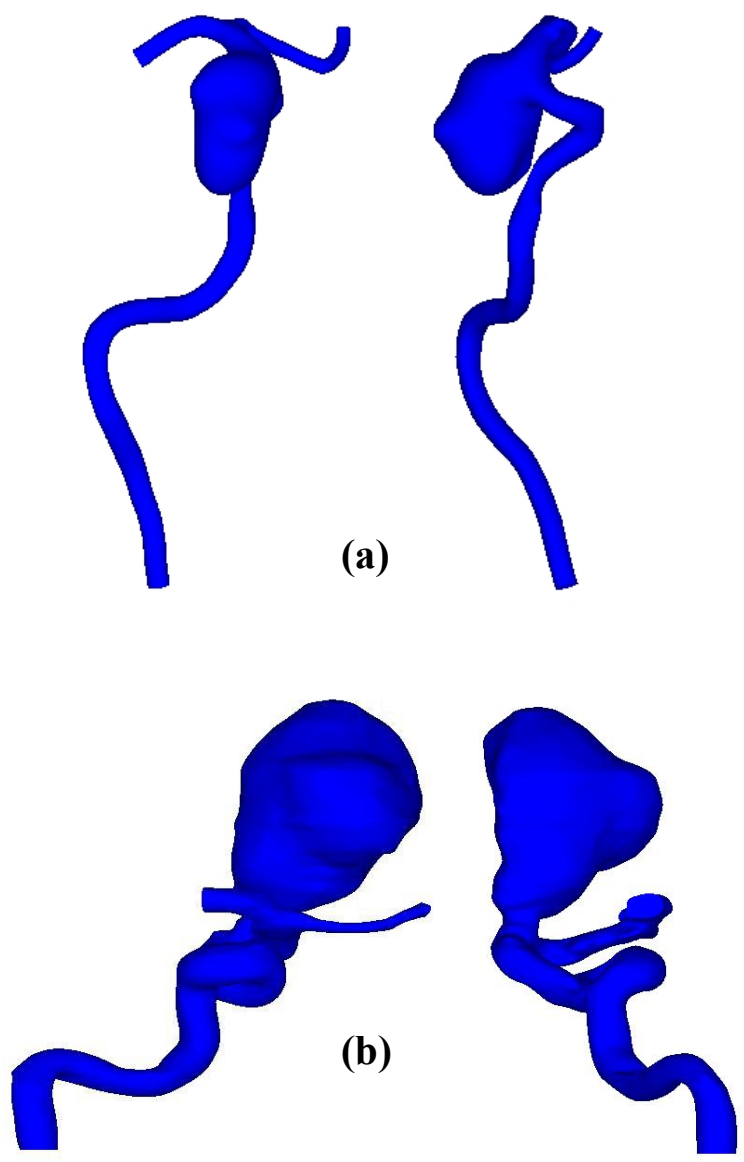

Figure 3.3: (a) Aneurysm-A - anteposterior (top left) and lateral (top right) views; (b) Aneurysm-B xy view (bottom left) and yz view (bottom right). 
Some measuring of certain regions was performed to compare with the old reference values obtained during the previous corroboration method. The new values do not differ much with the ones found in references. The results show better values than the ones obtained by Corcoran's method (Corcoran et al, 2003).

Although both aneurysms A and B are classified as saccular aneurysms, aneurysm-B shows a neck constriction at the entrance to the aneurysm, while aneurysmA shows growth directly from the side wall of the vessel with no defined neck. Aneurysm-A's geometry has a total volume of $7.47 \times 10^{-6} \mathrm{~m}^{3}$ of which, $4.5 \times 10^{-6} \mathrm{~m}^{3}$ or $60 \%$ belongs to the actual aneurysm section. The aneurysm-A has an inlet diameter of 4.2 $\mathrm{mm}$ approximately and $3.93 \mathrm{~mm}$ and $2.76 \mathrm{~mm}$ diameters at the large and small artery outlets respectively. While aneurysm-B's geometry shows a similar total volume of $7.42 \times 10^{-6} \mathrm{~m}^{3}, 76 \%$ or $5.65 \times 10^{-6} \mathrm{~m}^{3}$ of it resides in the aneurysm section with only $24 \%$ of the volume corresponding to the arterial sections. The inlet diameter of aneurysm-B is $4.72 \mathrm{~mm}$ and the outlet diameters are 3.84 and $2.18 \mathrm{~mm}$ for the large and small artery respectively.

\subsubsection{Mesh Properties}

The reconstructed geometries were exported to GAMBIT and meshed using tetrahedral volumes to generate a CFD mesh. Triangular and hexahedral elements were used to mesh the surface and volumetric elements. Steinman et al (2003) performed a grid dependence study on aneurysm-A and found 213,000 quadratic tetrahedral finite elements $(311,000$ nodes) to be sufficient for grid convergence. The grid used in the 
current study for aneurysm-A is finer than the one used in (Steinman et al, 2003) and is believed to be adequate to obtain grid independent results. Grid dependence study is also performed in the current work on aneurysm-B. Table 3.1 shows the number of cells summary used for both geometries with the aneurysm to carry out the dependence study. The mesh is refined about three times to estimate the error of the major flow variables and WSS due to the grid resolution. The procedure for estimation of discretization error, suggested by ASME Journal of Fluids Engineering, is applied at various representative locations along the aneurysm and arterial geometry.

Table 3.1: $\quad$ Summary of the grid resolutions and numerical schemes used for both aneurysms geometries

\begin{tabular}{|c|c|c|c|c|}
\hline \multirow{2}{*}{ Geometry } & \multirow{2}{*}{$\begin{array}{c}\text { Order of } \\
\text { discretization } \\
\text { error }\end{array}$} & \multicolumn{3}{|c|}{$\begin{array}{c}\text { Grid Resolution } \\
\text { (number of cells) }\end{array}$} \\
\cline { 3 - 5 } & & Coarse & Medium & Fine \\
\hline Aneurysm-A & $1^{\text {st }} \begin{array}{c}\text { and 2 } \\
\text { order }\end{array}$ & 464,453 & $1,256,704$ & $3,476,331$ \\
\hline Aneurysm-B & $\begin{array}{c}1^{\text {st }} \text { and 2 } \\
\text { order }\end{array}$ & 705,882 & $1,968,263$ & $4,784,918$ \\
\hline
\end{tabular}

FLUENT software is used to carry out the simulations solving the Navier-Stokes equations for unsteady laminar flow along with a scalar transport equation. The use of a scalar would emulate the contrast agent injected during the angiography as in the clinical study. 


\subsubsection{Fluid and Flow Properties}

A person has about 4 to 6 liters of blood depending on the body size. From this total volume, 52 to $62 \%$ is plasma and the remaining 38 to $48 \%$ is composed of various blood cells. Plasma is the liquid fraction of the blood and is the transport medium. This liquid portion of the blood is composed of approximately $92 \%$ water, with the remaining being organic and inorganic solute dissolved in water. The blood cells or formed elements are suspended in plasma. There are three types of blood cells: Erythrocytes or red blood cells, Leukocytes or white blood cells and Thrombocytes or platelets (Pardridge, 1998). Blood viscosity is 3 to 4 times thicker than the water, strongly dependent on three factors: hematocrit, temperature, and the flow rate. The first factor or hematocrit is a blood portion occupied by the red blood cells. An increase of $50 \%$ of the erythrocyte volume fraction would raise the blood viscosity about $100 \%$. The temperature also affects the blood viscosity. If the temperature decreases, the viscosity increases. Therefore, the second factor would increase approximately by $2 \%$ for each degree of Celsius decrease in temperature. Finally, the flow rate has a significant effect on the viscosity. This third factor can significantly increase the viscosity due to the increment of cell-to cell and protein-to-cell adhesive interactions at very low flow rates (circulatory shock). These interactions cause the erythrocytes to remain close to each other, increasing the blood viscosity.

As mentioned before, water represents the major component $(92 \%)$ of the blood plasma. Therefore, in the present simulations, water is used exclusively to characterize the blood composition due to the complexity to model particles in suspension (blood cells) and its functions in the cardiovascular system (convection transport, control 
system, and body temperature regulation). A comparative table (Table 3.2) is presented below to show the main properties between blood and water.

Table 3.2: $\quad$ Comparative properties between the blood and water

\begin{tabular}{|c|c|c|}
\cline { 2 - 3 } \multicolumn{1}{c|}{} & Blood & Water \\
\hline Density $\left(\mathrm{Kg} / \mathrm{m}^{3}\right)$ & 1060 & 998.2 \\
\hline Viscosity $(\mathrm{Kg} / \mathrm{ms})$ & $3.2 \times 10^{-3}$ & $1.003 \times 10^{-3}$ \\
\hline $\begin{array}{c}\text { Reynolds number } \\
\text { Aneurysm-A/B }\end{array}$ & $1120 / 1320$ & $3200 / 3600$ \\
\hline Composition & $\begin{array}{c}\text { Plasma (55\%) and several } \\
\text { blood cells (45\%): erythrocytes } \\
\text { (red blood cells) } \\
\text { Leukocytes (white blood cells) } \\
\text { and thrombocytes (platelets) }\end{array}$ & $\mathrm{H}_{2} \mathrm{O}$ \\
\hline
\end{tabular}

In order to visualize the aneurysm in the patient's body, a contrast agent is used during the rotational angiography as mentioned before. The physical properties for the contrast agent named Visipaque (Iodixanol) were taken from General Electric Healthcare indications (GE Healthcare of Visipaque Injection) and dosage manual and the pharmacopeia manual (Martindale, 1996). The diffusivity of the solute is calculated to emulate the contrast agent by Eqn 3.1 using Einstein-Stoke formulation (Cussler, 1997)

$D=\frac{K_{B} T}{f}=\frac{K_{B} T}{6 \pi \mu R_{0}}$

where $\mathrm{K}_{\mathrm{B}}$ is a quantity known as Boltzmann's constant, $\mathrm{T}$ is the temperature, $f$ is the friction factor and $\mu$ is the dynamic viscosity of the solvent. $R_{0}$, the molecular radius of the solute, is in the range of $0.65-1.05 \mathrm{~nm}$ (Schwuchow et al, 2002). This gives us a 
diffusivity of $2.52 \times 10^{-10} \mathrm{~m}^{2} / \mathrm{s}$. The diffusion coefficient $(d)$ required by the scalar equation can thus be calculated as $d=D^{*} \rho_{\text {Sol }}$ where $\rho_{\text {Sol }}$ is the density of the solvent equal to $1000 \mathrm{~kg} / \mathrm{m}^{3}$.

The flow is assumed to be pulsating with a waveform (see Figure 3.4) similar to that used by Ku's investigation ( $\mathrm{Ku}$ et al, 1985). The fluid is assumed to be water, an incompressible Newtonian fluid. The resulting average and peak flow rate is $234 \mathrm{~mL} / \mathrm{min}$ and $798 \mathrm{~mL} / \mathrm{min}$, respectively, during a period of $0.7 \mathrm{sec}$. The flow is assumed to be laminar, with the Reynolds number remaining below 2,100 except during systole peak when aneurysm-A and B show a Reynolds number of 3,200 and 3,600 respectively.

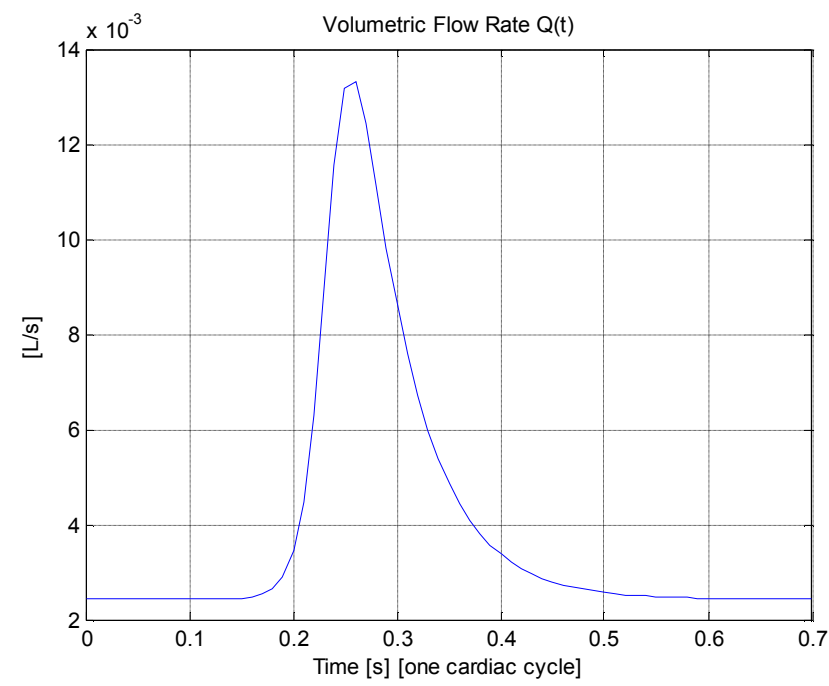

Figure 3.4: Inlet flow waveform approximation for one cardiac cycle (Ku et al, 1985)

Hence, laminar flow assumption is somewhat arbitrary, but it is the usual assumption employed by researchers in the literature. This assumption is made even though the values calculated at the systolic peak pass the critical Reynolds number; the real dimensionless number would be decreased due to the blood viscosity. 
Another dimensionless parameter (Womersley number) is also calculated in order to describe the ratio of transient inertial forces to viscous forces. The values for such dimensionless number are 6.32 and 7.05 for aneurysm $\mathrm{A}$ and $\mathrm{B}$, respectively. If the Womersley number exceeds 10, the frequency of pulsations is large and the velocity profile is relatively flat. Then, the mean flow lags pressure gradient by about 90 degrees for laminar and incompressible oscillatory flows. Therefore, the mean flow and the pressure gradient should be nearly in phase during a cardiac cycle and it would be shown in the results section for both geometries. For the scalar representing the dispersion agent, the boundary conditions represent an injection at the peak systole $(t=0.25 \mathrm{~s})$ for a total of 1.8 seconds. The duration of the contrast agent injected is the same when the angiography was performed; however, the amount of the contrast agent differs in the simulation because it was originally introduced by a needle. The needle is located in the neck so at the time it reaches the aneurysm, it has mostly diffused in the blood. During the simulation, the passive scalar gets inside all over the cross section of the parental inlet. Simulations are run for a total of 10-12 cardiac cycles at which time the most of the contrast agent is flushed out of the system.

\subsubsection{CFD Simulation Parameters}

In order to carry out the simulations, certain assumptions are made to approximate the blood flow in arteries such as:

- Rigid walls: this assumption is made due to the lack of information of the wall compliance in diseased arteries. The aneurismal geometry-A was obtained from 
Dr. Steinman, who has performed previous simulations in this geometry. However, the aneurismal geometry-B was from Dr. Rai, using a rotational angiogram which does not have the capacity to capture the wall movement.

- Fully developed flow is assumed at the entrance of the parental inlet. The shape of the velocity profile would depend on the arterial configuration preceded by the section obtained by the rotational angiogram.

- Blood assumed to be liquid water.

- Laminar flow is assumed during all the cardiac cycle.

- The volumetric flow is adjusted as Ku's investigation (Ku et al, 1985) due to the lack of information of the waveform for both geometries.

- Both geometries involve an arterial bifurcation downstream of the aneurysm thus presenting dual flow outlets. The effect of the boundary condition at the outlet on the flow patterns is recorded initially by using a "pressure-outlet" boundary condition at each of these outlets and then recalculating the flow using an "outflow" boundary condition. When the pressure outlet BC is chosen, zero gauge pressure is set to carry out the simulation. Once this simulation has finished, the mass flow ratio is calculated to be imposed in a new simulation using an outflow $\mathrm{BC}$. Special care has to be given to the boundaries during the simulations because the human body does not present zero pressure outlets.

- The momentum equation is solved using a first-order upwind and second-order (QUICK) discretization schemes and the scalar transport equation is solved using second order schemes. 
- The criterion of convergence is $10^{-3}$ for both geometries (with and without the aneurysms).

\subsection{Removal of the Aneurysm}

\subsubsection{Aneurysm-A geometry with no aneurysm}

In order to study the hemodynamic factors that may trigger the occurrence of aneurysms, it is necessary to go back in time and study the flow when the artery was healthy. Consequently, the use of 3D Reshaper (Technodigit, Gleizé, France) software allows the removal of the aneurysms and its reconstruction to a healthy artery. As an initial step, the diameters of the artery nearby (before and after the aneurysm) are used as reference for the reconstruction process. These diameters are used to maintain the artery diameter of the reconstructed region. Only a small region of the vessel is not affected by the disease, so this section will provide guidance to the restoration procedure. Over this small region, several rings with similar diameters are built, as suggested by Himeno's investigation (Himeno, M., 2007). These rings are then connected and smoothed. The new geometry without the aneurysm is exported to GAMBIT and re-gridded. CFD simulations are carried out using similar procedure described before. An initial simulation using a pressure outlet $\mathrm{BC}$ is carried out to find the mass flow rates through each of the outlets. The results obtained are imposed in a new simulation using the outflow BC.

This new geometry is analyzed three locations in the region of interest (where the aneurysm was initially located as shown in Figure 4.6). These three locations correspond to cross sections placed before (BA), in situ (IS), and after (AA) the aneurysm was 
originally situated. The study of the flow characteristics at these locations is performed with focus on the possible hemodynamic factors that may cause the wall deformation, triggering the aneurysms. A grid dependence study is performed using three grid resolutions to estimate the error in velocity calculations.

\subsubsection{Aneurysm-B geometry with no aneurysm}

In a similar manner to Aneurysm-A, the aneurysm removal process is performed on Aneurysm-B by using 3DReshaper application. Again, the arterial diameter in the vicinity of the cerebral aneurysm location is used as a reference to reconstruct the lumen vessel. In this particular case, once the aneurysm is removed, the empty area represents a small region due to the neck, which connects with the aneurysm. The opposite side of the artery (where the aneurysm is located) will provide topographical information of the arteries facilitating the reconstruction process. Compared to Aneurysm-A, this geometry resulted in an easier process due to the few rings needed to refill the hole. The new geometry (see Figure 4.6) is exported to GAMBIT and the grid size is redefined before the CFD simulations are carried out. As explained before, the FLUENT simulation used the same parameters as geometry-A. The pressure outlet boundary condition is initially imposed to determine the mass flow ratio before a new simulation using outflow boundary condition is started. Again, three cross sections are made at before (BA), in situ (IS), and after (AA) the aneurysm was located, to analyze the flow characteristics that may influence the formation of aneurysm. 
Table 3.3: Summary of the grid resolution for both geometries without the aneurysm

\begin{tabular}{|c|c|c|c|}
\multicolumn{1}{c|}{} & \multicolumn{3}{c|}{ Grid Resolution } \\
\cline { 2 - 4 } \multicolumn{1}{c|}{} & \multicolumn{2}{c|}{ (Number of computational cells) } \\
\hline Geometry & Coarse & Medium & Fine \\
\hline A & 270,668 & 890,511 & $2,764,893$ \\
B & 435,704 & $1,169,504$ & $3,183,674$ \\
\hline
\end{tabular}

Table 3.3 shows the three different grid resolutions used to perform the simulations in the geometries without the aneurysm. The simulations for these geometries were carried out by using only a second order "QUICK" discretization scheme for the momentum equation. A grid dependence study was also performed to estimate the discretization error of the velocity profiles and the WSS at different locations through a method known as grid convergence index (GCI).

\subsection{Grid Convergence Index (GCI)}

An estimation of the discretization error in the velocity and WSS profiles was made using the GCI method based on the Richardson extrapolation (RE). Further information on GCI can be found in Celik's et al (2004) study. Initially, the representative cell size of each mesh is calculated using:

$h_{i}=\frac{1}{N}\left[\sum_{i=1}^{N}\left(\Delta V_{i}\right)\right]^{1 / 3}$ 
where $\mathrm{N}$ is total number of cells and $\Delta \mathrm{V}_{\mathrm{i}}$ is the volume of each computational cell. Using a grid refinement factor $\mathrm{r}=\mathrm{h}_{\text {coarse }} / \mathrm{h}_{\text {fine, }}$, the apparent order of the method $\mathrm{p}$ is calculated by solving the system of nonlinear equations

$$
\begin{aligned}
& p=\frac{1}{\ln \left(r_{21}\right)}|\ln | \varepsilon_{32} / \varepsilon_{21}|+q(p)| \\
& \varepsilon_{i j}=\phi_{i}-\phi_{j} \\
& q(p)=\ln \left(\frac{r_{21}^{p}-s}{r_{32}^{p}-s}\right) \\
& s=1 . \operatorname{sign}\left(\varepsilon_{32} / \varepsilon_{21}\right)
\end{aligned}
$$

The grid is indexed such that $\mathrm{h}_{1}<\mathrm{h}_{2}<\mathrm{h}_{3}$ from the approximate errors $\left(\mathrm{e}_{\mathrm{a}}^{21}=\mid \Phi_{\text {fine }^{-}}\right.$ $\left.\Phi_{\text {coarse }} \mid\right)$.

The fine grid convergence index is calculated using

$$
G C I_{\text {fine }}^{21}=\frac{1.25 e_{a}^{21}}{r_{21}^{p}-1}
$$

This methodology has a particularity when the error is estimated. If at least two profiles cross at any point, the calculation of the error fails. Further investigation is needed to improve this methodology at these inflection points. 


\section{Results}

\subsection{Preliminary Study}

Several factors affect the flow characteristics in the arteries with aneurysms. A pipe geometry is built to carry out preliminary simulations to understand the phenomena. This geometry would facilitate the study of hemodynamic factors that play an important role in the growth and development of the side-wall aneurysms. Real aneurysm's geometries demostrate very complex flows. This first approach would elucidate these possible factors, influencing the occurrence in cerebral aneurysms. This experiment shows the importance of the geometric configuration and the sensibility of the grid resolution to predict the flow characteristics in pseudo-aneurysm geometries. Two pipes are created and bended 30 and 60 degrees to emulate the arterial curvature. These geometries have a constant diameter similar as Aneurysm-B geometry $(4.7 \mathrm{~mm}$ ) (see section 3.2.2). The region of interest corresponds to the bent zone of the pipe and where this preliminary study is focused. The construction, meshing, and boundary condition definition are performed in GAMBIT and the CFD calculations are carried out in FLUENT software. Similar grid resolution is applied for both geometries, which is about 550,000 cell elements.

Like the human cardiac cycle, the flow is assumed to be pulsating with a waveform (see Figure 3.1) similar to the one used by Mantha's investigation (Mantha et al, 2006), who claims that the flow patterns and stagnation regions are independent of changes in flow rates. The volumetric flow waveform is imposed at the inlet and a pressure outlet boundary condition is imposed at the outlet of the pipe. The fluid is assumed to be water, 
an incompressible Newtonian fluid. The flow cycle is simulated for the present case and the flow characteristics are analyzed in the same three cross sections perpendicular to the flow direction. These planes are located on the onset (Plane-1), on the middle (Plane-2), and at the end (Plane-3) of the bend in the geometry as shown in Figure 4.1. Laminar flow is assumed for the cardiac cycle, although turbulent flow may occur at the systolic peak.

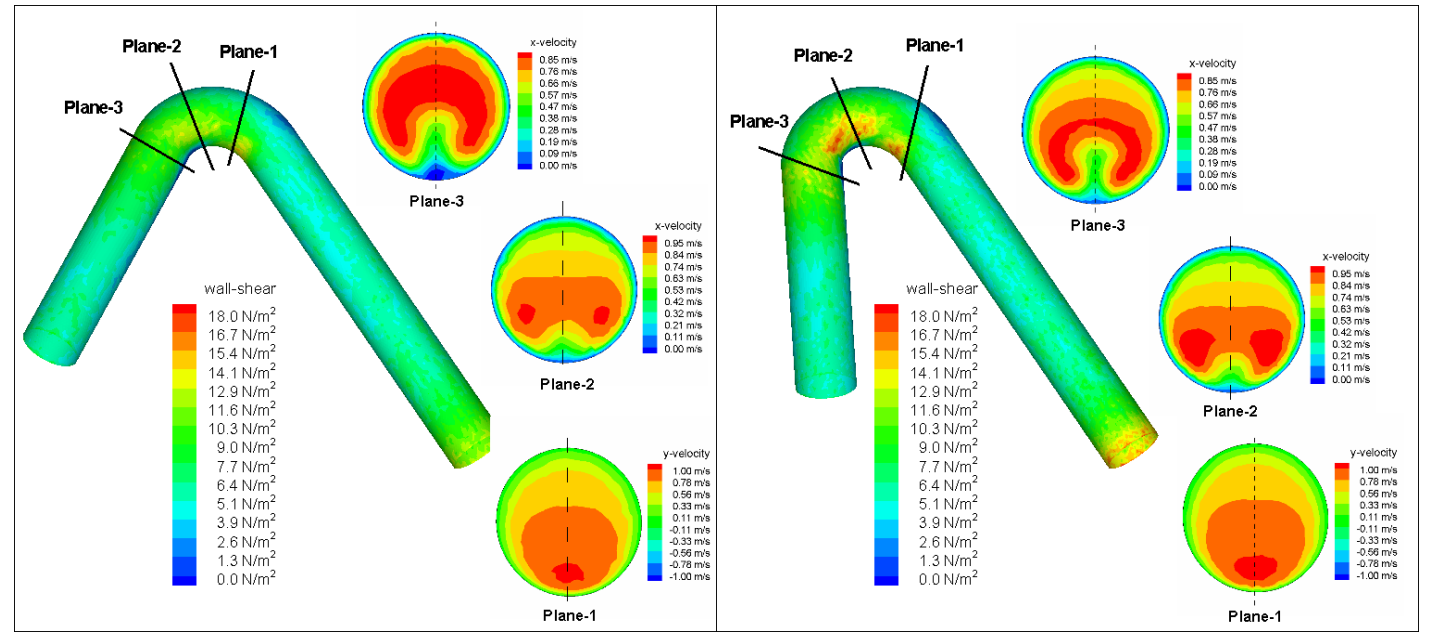

Figure 4.1: WSS comparison and flow axial velocities using a pipe bent with 30 degrees (left) and 60 degrees (right) with respect to the horizontal.

Once the simulations are complete, the results are analyzed in the three defined planes. Figure 4.1 shows the results of the WSS contours between different configurations of the bent pipes. The maximum values for these geometries are 14.74 $\mathrm{N} / \mathrm{m}^{2}$ and $19.07 \mathrm{~N} / \mathrm{m}^{2}$ for 30 and 60 degree angle-bended pipes respectively during the systole peak. The results show that when the bent angle is higher, the WSS magnitude increases in the convex region (inner area) of the curvature. Analyzing the $\mathrm{x}$ and $\mathrm{y}$ WSS components (Figure 4.2), the flow impingement contributes to the WSS, causing an increase in the magnitude of WSS. The vortical flows at plane-3 reaches the outer bend on the 30 degrees bent pipe. On the contrary, the flow at the same plane for the 60 
degrees bent pipe does not reach the inner concave region as shown in Figure 4.3. This flow impingement at the 30 degrees bent pipe will contribute to higher WSS at plane-3 as seen in Figure 4.2. The WSS at this location represents an increment of $40 \%$ (about 5 $\mathrm{N} / \mathrm{m}^{2}$ ) in the magnitude of the $\mathrm{x}$ component.
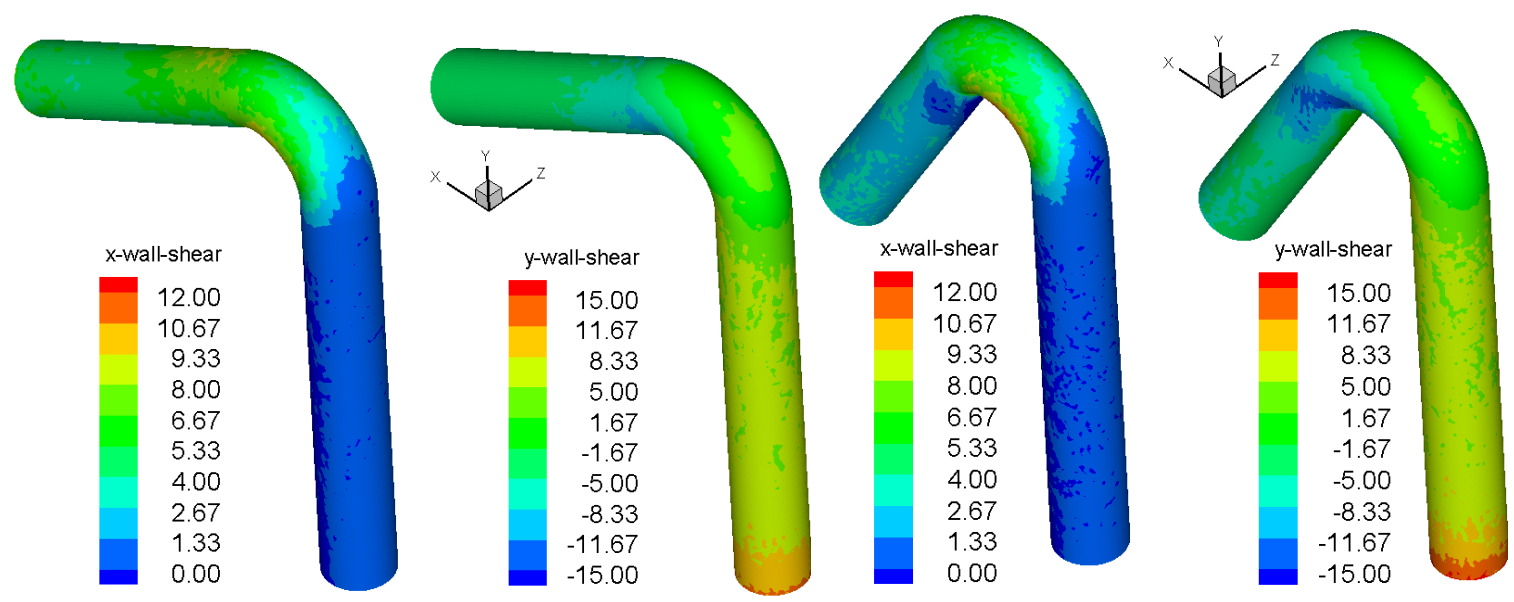

Figure 4.2: WSS components in the $x$ and $y$ direction for the bent pipe with 30 degree (left) and with 60 degree (right) with respect to the horizontal

Figure 4.3 shows velocity vectors of the cross sectional planes for both geometries with the contours colored by the dynamic pressure. Centripetal forces are found at the convex region following the direction of the pipe bend. As seen in Figure 4.3, the primary flow manifests a two pressure regions: the fastest speed (centrifugal force) located in the concave region (outer section of the curvature pipe), driven by the low pressure, and the slower speed flow located in the convex region (inner section of the pipe curvature), contributes to the high pressure. Secondary flows are also observed in the boundary layer. 

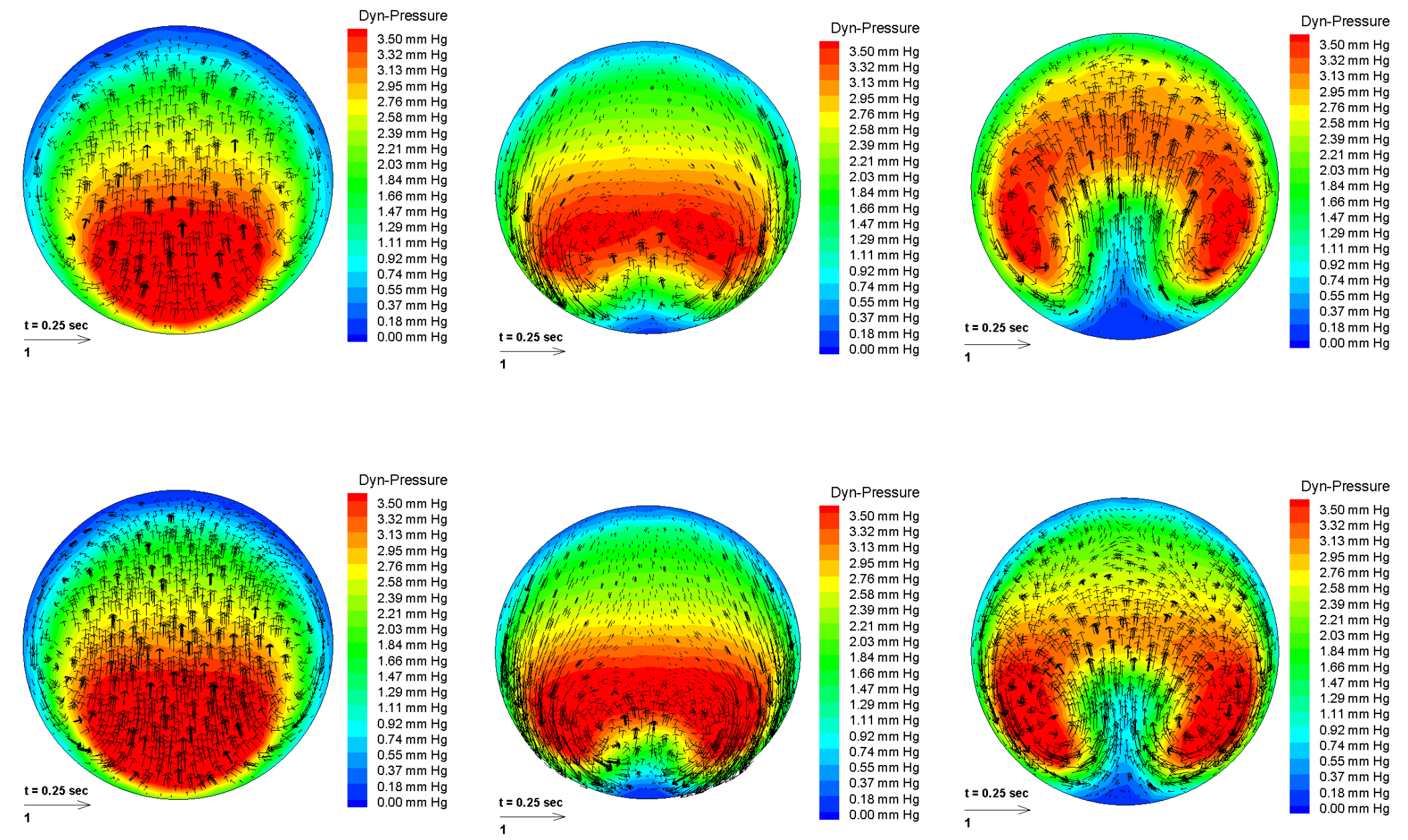

Figure 4.3: Velocity vectors colored by the dynamic pressure contours ( $\mathrm{mm} \mathrm{Hg}$ ) at different cross sections for a bent pipe with 30 (top) and 60 (bottom) degrees at the systolic peak: Plane-1 (left), Plane-2 (middle), and Plane-3 (right) 
The secondary flows move up towards the concave region where recirculation vortices get mixed with the primary flow. This effect may erode the outside region of the pipe bend. Figure 4.3 also shows the plane- 1 and plane- 2 where the secondary flows are observed in both geometries with very similar flow characteristics.

A further study is performed using a spherical bulge created on both bent pipes to simulate an aneurysm. The bulge (sphere) is located in the same position for both the pipes. The sphere and the bent pipe are connected smoothly so they do not have sharp edges which will have a strong effect on the flow characteristics. These geometries are re-meshed using a similar number of cell elements (approximately 850,000 computational cells). Figure 4.4 shows the WSS changes of the geometries with a bulge. The maximum value of WSS is located upstream of the connected region between the sphere and the pipe for both the geometries with a magnitude of $29.55 \mathrm{~N} / \mathrm{m}^{2}$ and 24.2 $\mathrm{N} / \mathrm{m}^{2}$ for 30 and 60 degrees pipes respectively. The magnitude of the WSS increases in the upstream region of the bulge when compared to the pipe without pseudo-aneurysm. This increment is due to the changes in the flow inside the pipe.

In order to quantify the effects of grid resolution, the pipe bent at 60 degrees is chosen to perform a grid sensibility study. Simulations with two different grid resolutions are completed made with the same initial set-up, using 55,799 cells and 453,181 cells for a coarse and a fine grids respectively. This study will yield an estimate of the error in velocity profiles error and consequently the WSS error in the CFD calculations. 


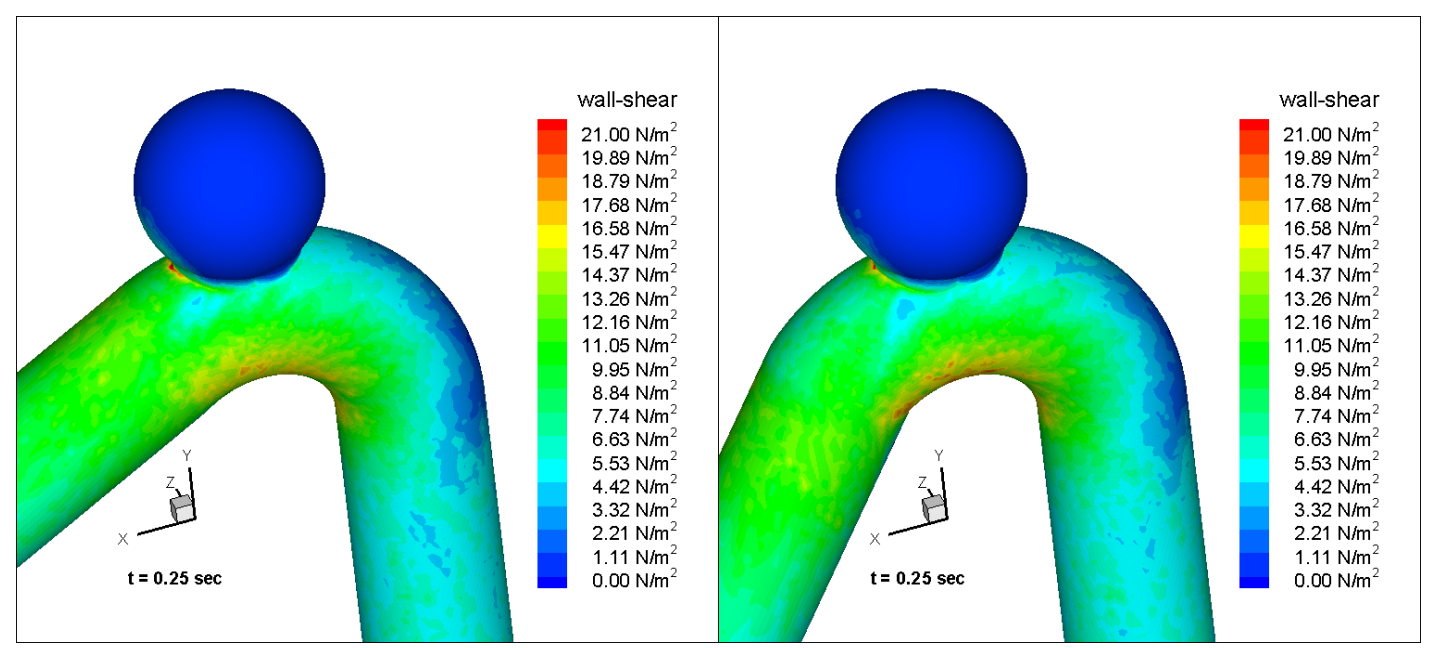

Figure 4.4: WSS contours of pseudo-aneurysms with 30 (left) and 60 (right) degrees angle bended pipe

The resolution of the boundary layer near the wall plays a significant role in WSS calculation. Resolution of the boundary layer near the wall is very sensitive to the grid resolution and the nodes distribution in the mesh. WSS is calculated as the normal velocity gradient at the wall in laminar flow as:

$\tau_{w}=\mu\left(\frac{d v}{d n}\right)$

Figure 4.5 shows the location of the maximum wall shear stress in the coarse grid (left) and fine grid (right) and their respective magnitude is $8.7 \mathrm{~N} / \mathrm{m}^{2}$ and $15.9 \mathrm{~N} / \mathrm{m}^{2}$. This figure also shows the comparisons between the axial velocities in each of the three defined cross sections. As expected, the fine grid shows better details of the recirculation vortices (secondary flows) at the pipe bent. While the coarse grid fails to resolve the recirculation vortices accurately and shows some flow reversal nears the inner wall. 


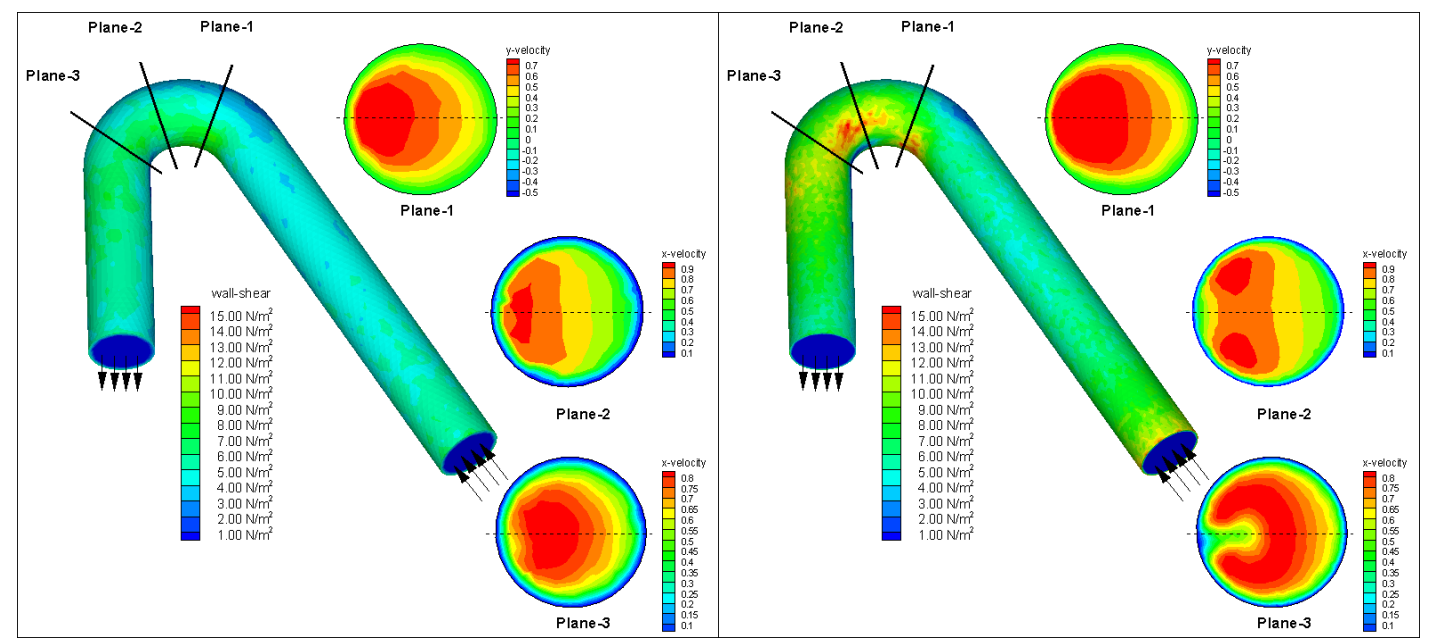

Figure 4.5: WSS comparison and flow profiles obtained using the coarse grid (left) and the fine grid (right). The axial velocity profiles are compared at the representative lines (dashed lines) on each of the three planes.
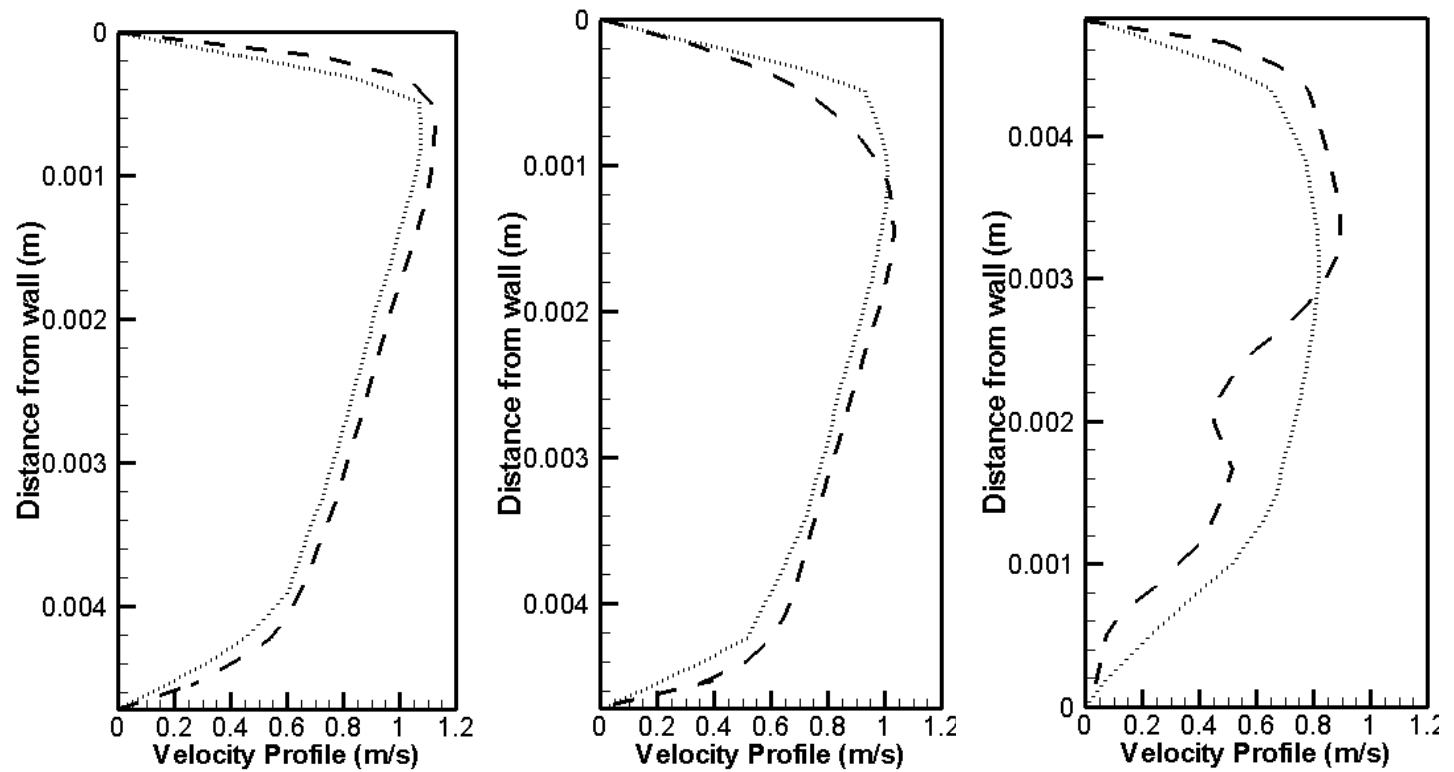

Figure 4.6: Velocity profiles comparison and the near wall velocity profiles on three representative planes at the systole peak using the coarse grid (dot line) and the fine grid (dash line): Plane-1 (left), Plane-2 (middle), Plane-3

(right).

The velocity profiles across the diameter of these planes are shown in Figure 4.6.

The velocity profile using fine grid at plane-3 indicates decelerated flow in the convex region (inner zone) and accelerated flow due to the centrifugal force at the concave region (outer zone) of the pipe. Centrifugal forces occur when the flow enters a curved 
region in which the fluid is pushed away from the center of curvature. The core region fluid with higher velocity moves toward the outer wall. The fluid returns along the wall in the direction of the inner wall as a consequence of the radial pressure gradient. The secondary vortices get mixed with the primary flow producing higher velocities near the outer wall; thus, the axial velocity is distortioned as shown in plane-3 of Figure 4.5. The recirculation vortices or secondary flows are developed on planes normal to the direction of the primary blood flow and impinge on the upper internal pipe wall. This repeated process over many years of the patients' lifetime may cause damage to the interior wall, triggering changes in the wall structure. Therefore, these results indicate that in a pipe flow the factors that may trigger degeneration of the wall structure are the flow impingement and secondary flows. Recirculation flow zones can also promote the changes in the wall structure due to the cyclic WSS observed in recirculation vortices in unsteady flows.

While the blood flow in arteries is more complicated than the bent pipe investigated, the flow characteristics mentioned above would contribute to the aneurismal development. These factors in actual/real geometries will be studied further in both real geometries with and without the aneurysm. Additionally, the WSS will also be considered in the present investigation. Flow simulations for aneurysm geometries A and B generated in section 3.0 are continued using parameters listed in section 3.1. 


\subsection{Aneurysms Geometries}

For Aneurysm-A, with the contrast agent injected at peak systole $(t=0.25 \mathrm{sec})$, almost immediate mixing of the fluid and contrast agent over the cross-section of the artery was found to occur. The contrast agent follows the flow reaching the aneurysm within one cardiac cycle. Similar behavior was found in the in-vivo simulation generated by Leonardo software (Rai, 2006).

The contrast agent fills the aneurysm and is flushed out within six cycles. The simulation is continued for twelve cardiac cycles, at which time there is very little contrast agent present in the aneurysm. The scalar concentration profiles (filled color) and the velocity vectors (in black) at different times, across the lateral (left) and coronal (right) cross-sections of the aneurysm-A are shown in Figure 4.7. The swirling pattern of the flow as it enters the aneurysm can be clearly seen in Figure 4.7. The wide neck opening of the aneurysm allows blood to flow in and out easily. This is, however, not the case for aneurysm-B (Figure 4.8), which shows the scalar concentration profiles and the velocity vectors for the aneurysm-B at different times. For aneurysm-B, the neck constriction prevents the bulk of the scalar (red filled contours) from reaching the top of the aneurysm. The swirling motion of the flow is, however, similar to the one seen for the aneurysm-A (Figure 4.7). The dynamic filling and flushing of the aneurysm with the contrast agent is best captured in a 3D video and is difficult to represent in a $2 \mathrm{D}$ format.

In addition to these representations of the contrast agent, four representative cross-sections are chosen and the results are presented in the form of velocity, pressure and scalar profiles across the artery's diameter in these cross sections. 


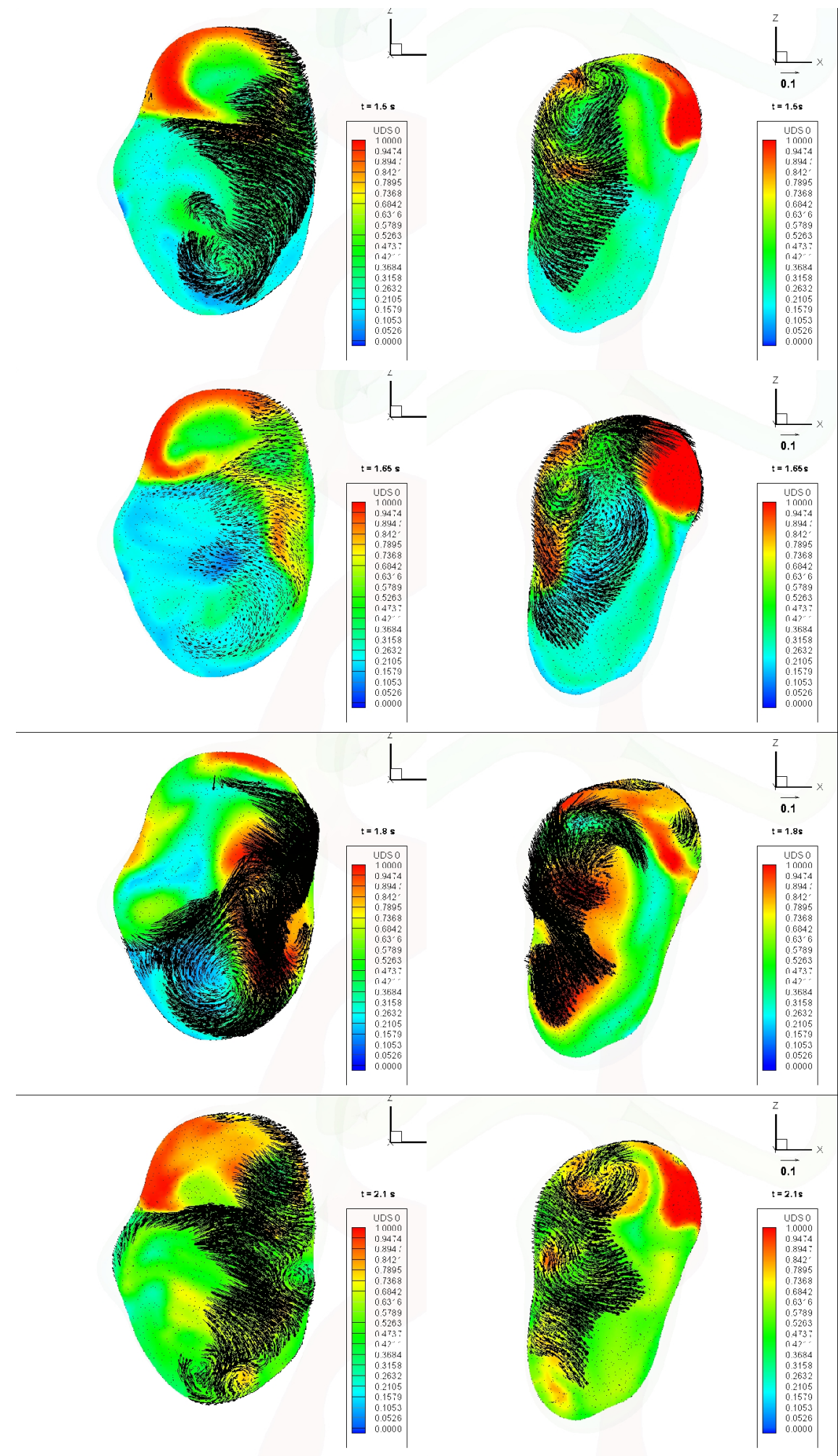

Figure 4.7: Snapshots of flow inside the Aneurysm-A, sagittal plane (left), coronal plane (right) at $\mathrm{t}=1.5,1.65,1.8$, and $2.1 \mathrm{sec}$ 


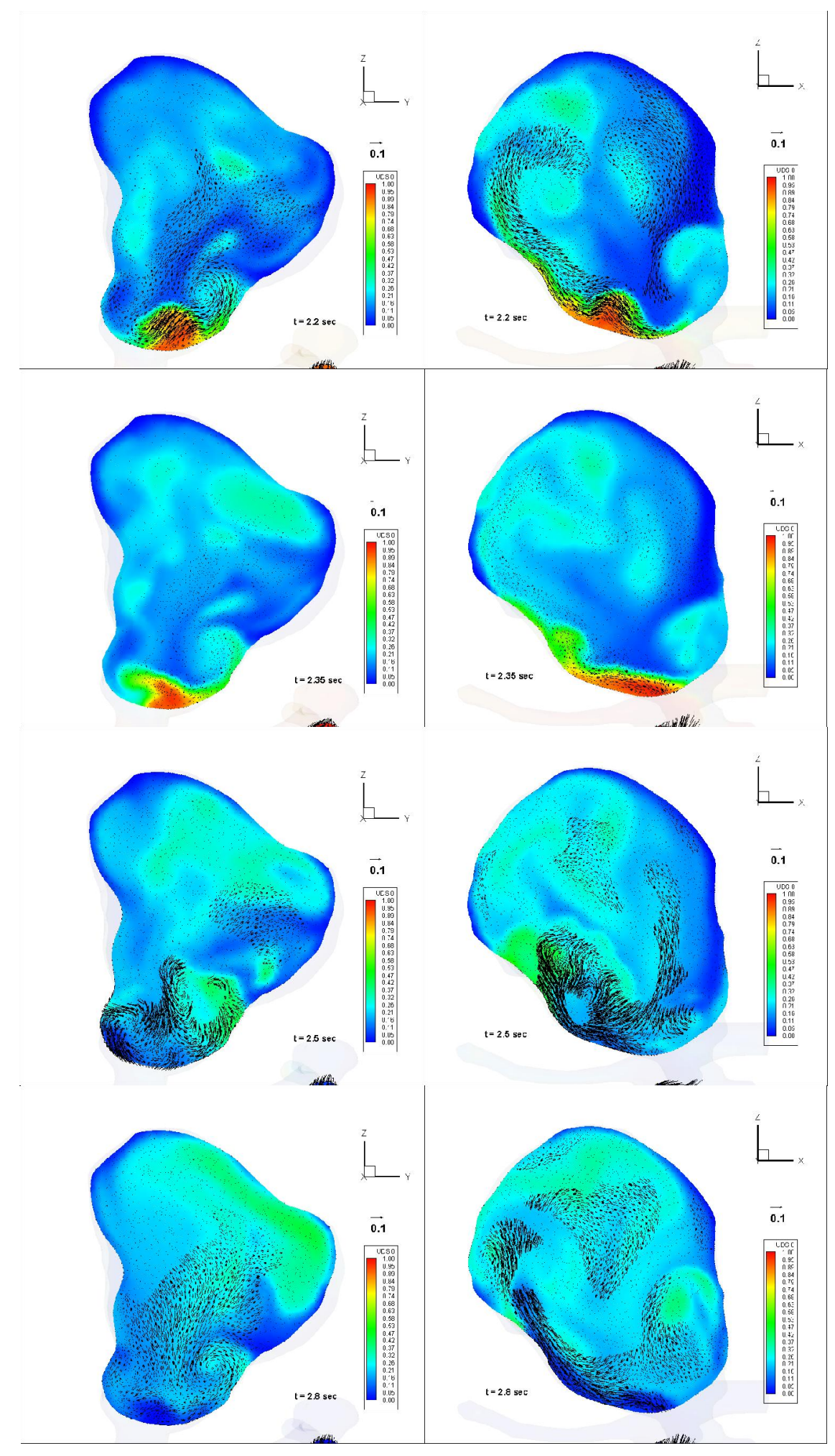

Figure 4.8: Snapshots of flow inside the Aneurysm-B, sagittal plane (left), coronal plane (right) at $t=2.2,2.35,2.5$, and 2.8 sec. 
These sections are named (a) entrance to the aneurysm (EA), (b) section before the bifurcation downstream of the aneurysm $(\mathrm{BF}),(\mathrm{c})$ the large artery outlet section $(\mathrm{O} 1)$ and (d) the small artery outlet section (O2), and are depicted in Figure 4.9 for both aneurysms. All these cross-sections are normal to the primary axial flow. The systole peak of the second and third cycles $(t=0.95$ and $t=1.65 \mathrm{sec})$, and the late diastole of the second and third cycles $(\mathrm{t}=1.40$ and $\mathrm{t}=2.10 \mathrm{sec})$ are taken as representative times for plotting the velocity profiles across each of these cross sections.

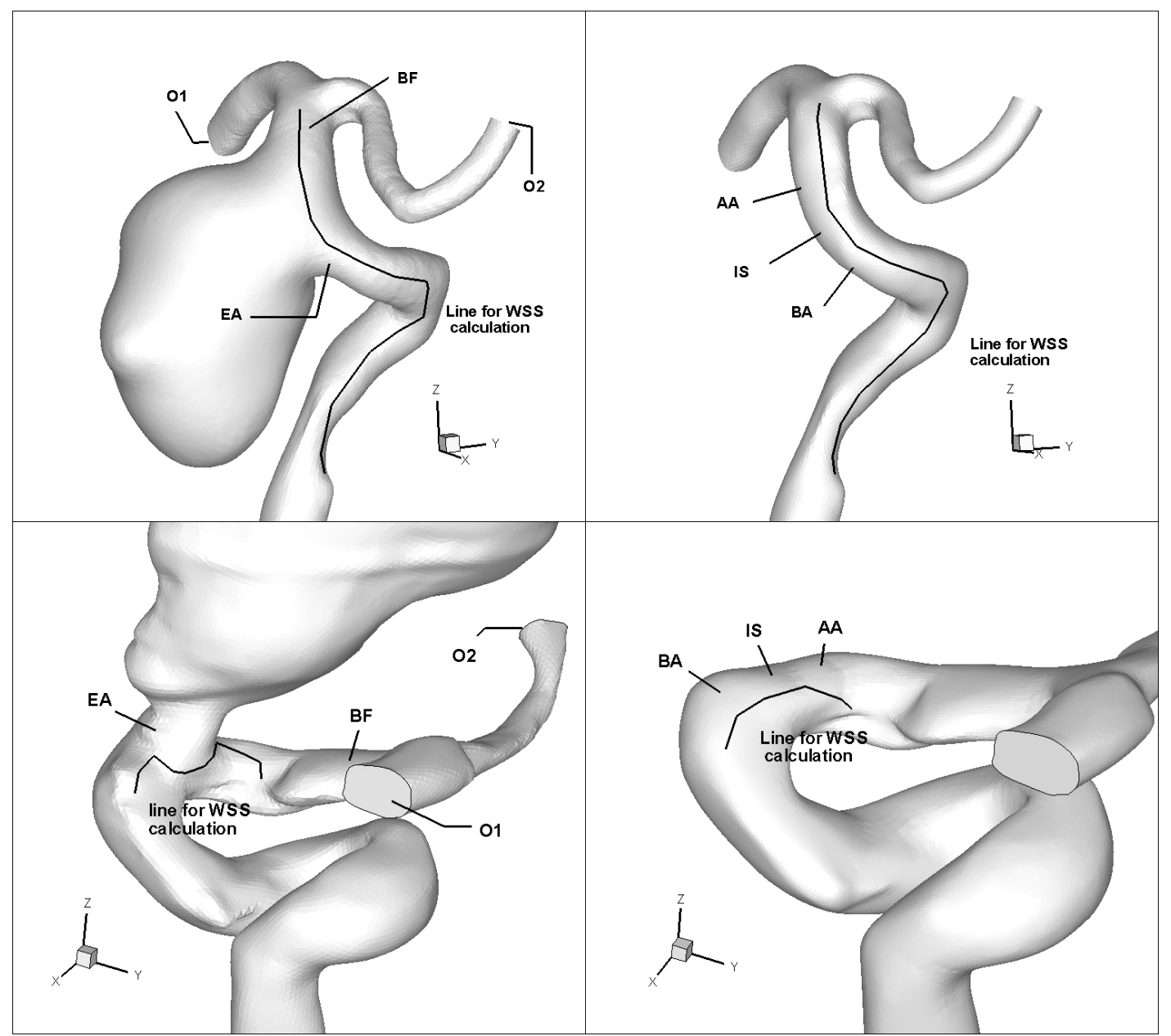

Figure 4.9: Location of the cross-sectional planes and a line traced for WSS calculations where results are presented for both geometries with and without the aneurysm (top two images for aneurysm-A and bottom two images for aneurysm-B) 


\subsubsection{Results for Aneurysm-A}

\subsubsection{Boundary Conditions for Aneurysm-A}

The pressure outlet boundary condition assumes a gauge pressure of $0 \mathrm{~Pa}$ at each of the outlets. The outflow boundary condition imposes a zero diffusive flux for all the flow variables while maintaining the overall mass flow rate. The outflow boundary condition needs an additional input of fractional mass flows through each of the outlets $\mathrm{O} 1$ and $\mathrm{O} 2$. These fractional values $(75 \%$ of overall mass flow thorough $\mathrm{O} 1$ and $25 \%$ through $\mathrm{O} 2$ ) are acquired from the values obtained using the pressure outlet boundary condition. The mass flow ratio for dual outlets can be calculated analytically (Taylor, 2008).

Ohm's law states that the current passing between two points is proportional to the potential difference and inversely proportional to the voltage drop. This formulation when applied to hydrodynamic calculations is known as Darcy's Law where the flow resistance or impedance can be written as

$Z=\frac{P}{Q}$

where $\mathrm{Z}$ is the instantaneous impedance for a transient flow, $\mathrm{P}$ is the pressure along the axial length of the artery, and Q is the volumetric flow rate along the axial length of the artery. Additionally, the mass is conserved at each junction and the pressure is continuous at the junction for the hydraulic analogy.

Figure 4.10 shows this comparison between these two different boundary conditions used during the cardiac cycle. The impedance waveforms for both boundary 
conditions used at a parental inlet (PI) cross section show similar trends, but different magnitudes. On the other hand, the impedance at the dual outlets $\mathrm{O} 1$ and $\mathrm{O} 2$ differ not only quantitatively but also qualitatively for both boundary conditions.

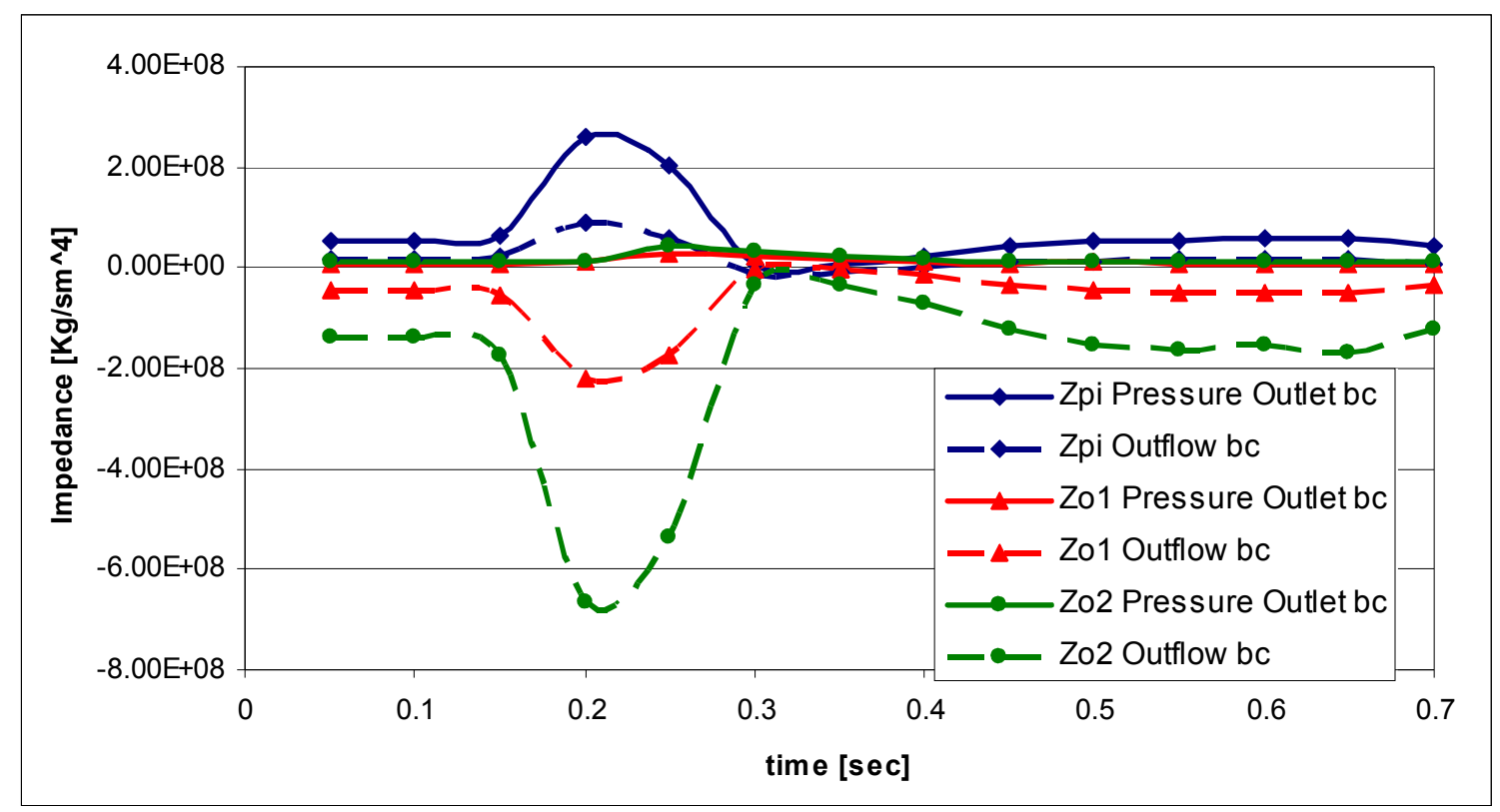

Figure 4.10: Impedance comparison between Pressure Outlet BC and Outflow BC at various locations during a cardiac cycle for Aneurysm-A

Figure 4.10 also shows that the impedance for the $\mathrm{O} 1$ and $\mathrm{O} 2$ cross sections for outlet boundary conditions have negative values during the whole cardiac cycle. The flow resistance at the outlets using pressure outlet boundary conditions sucks pressure from the system, while the negative flow resistance at the outlets adds pressure into the system for outflow boundary condition. This means that the flow resistant for the latter acts as a pressure source which is proportional to the volumetric flow rate passing through it.

According to the Hagen-Poiseuille law derived from Navier-Stokes's equations for circular cross sections fluids flows, the maximum velocity is located at the core of the pipe. However, arteries do not have perfect circular shapes, so the peak of the velocity 
would not necessary lie at the center of the outlet cross sections, due to bend and the diameter changing. In addition, the BF section which is upstream of the arterial bifurcation should start showing the flow division in the blood vessel. To observe all these characteristics, the flow is studied at the defined planes as mentioned before.

Figure 4.11 shows the velocity profile using different boundary conditions at the EA and BF cross sections. At the systole times (left in Figure 4.11), the maximum difference in velocity profile between the two boundary conditions is less than $30 \%$ and $10 \%$ for EA and BF cross sections respectively. This major difference using fine grid resolution in the peak-systole shows the importance of using the right boundary conditions which affect the flow behavior at the location upstream of aneurysm-A. This change in the profile may be crucial to simulate the blood flow entering and exiting the aneurysm. For example, this change at the EA cross section could play an important role in the calculation of the vortices inside the bulge which can mislead to premature hemodynamic conclusions. While during the late diastole, the maximum differences are approximately $5 \%$ and $15 \%$ for EA and BF respectively (right of Figure 4.11 ). At the peak of the deceleration phase of the BF cross section, the reflected wave may reach the aneurismal region, affecting the flow characteristics in this zone. The cause of the major difference of the velocity profile at the BF cross section is due to the mass flow ratio imposed corresponding to the one found for the systolic peak using pressure outlet $\mathrm{BC}$. This flow rate remains the same for all cardiac cycle periods during the simulation. The amounts found for the $\mathrm{O} 1$ cross section vary from $74.38 \%$ at the systole peak to $75.62 \%$ at the end-diastole when pressure outlet $\mathrm{BC}$ was used. 


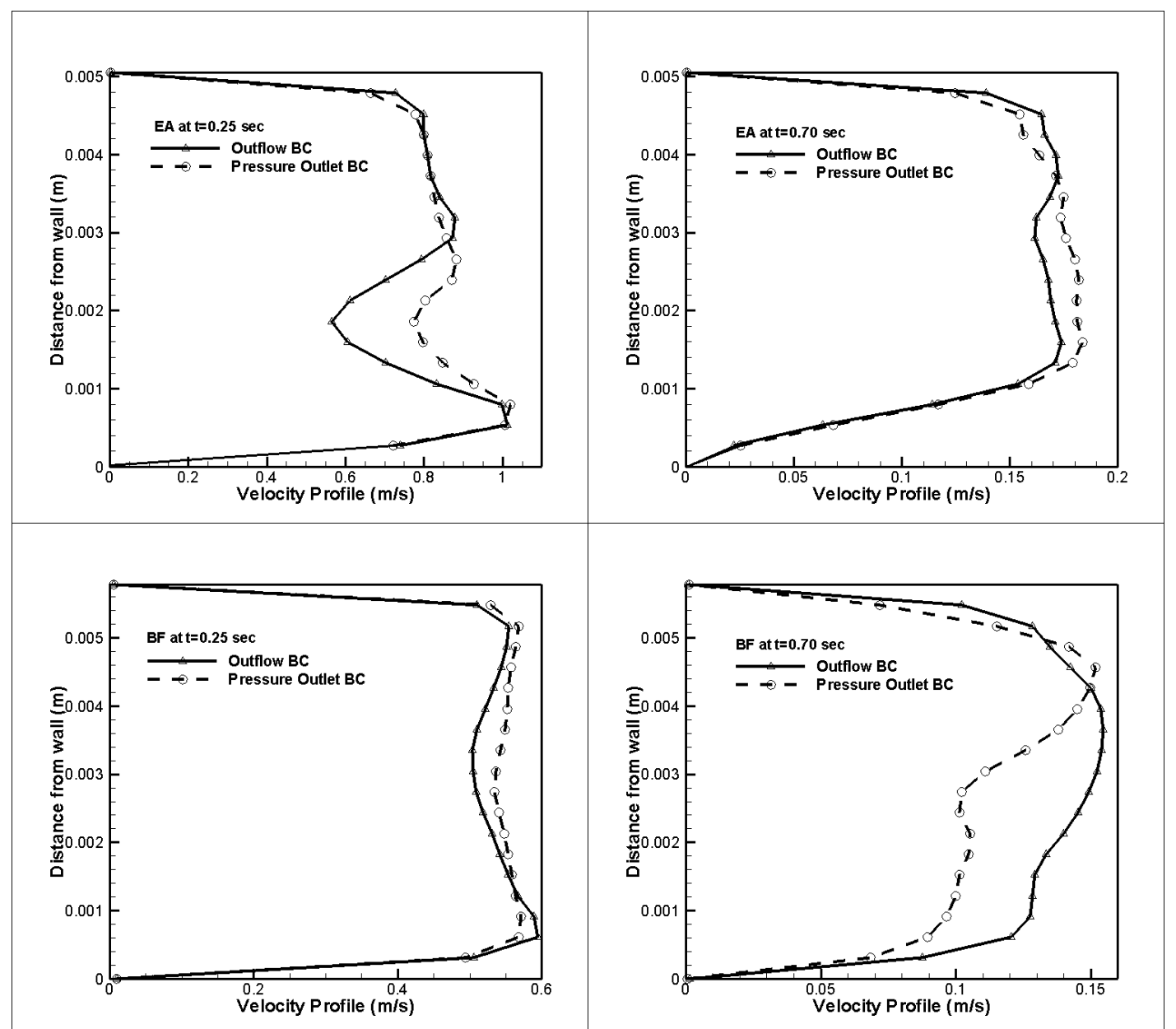

Figure 4.11: Velocity profiles at EA (top) and BF (bottom) cross sections at the systole peak (left) and late diastole (right) for Aneurysm-A using different boundary conditions

The high WSS would occur at the systole-peak, which many researchers claim to cause intracranial aneurysms. Therefore, the errors obtained at high velocities would have a small influence in the calculation of the WSS compared to the ones obtained at low velocities.

A similar comparison is performed to the outlets using a pressure outlet and an outflow BC. Figure 4.12 shows the velocity profiles using two different BC for O1 (top of Figure 4.12) and $\mathrm{O} 2$ (bottom of Figure 4.12) cross sections during the systole peak (left of Figure 4.12) and the late diastole (right of Figure 4.12). 


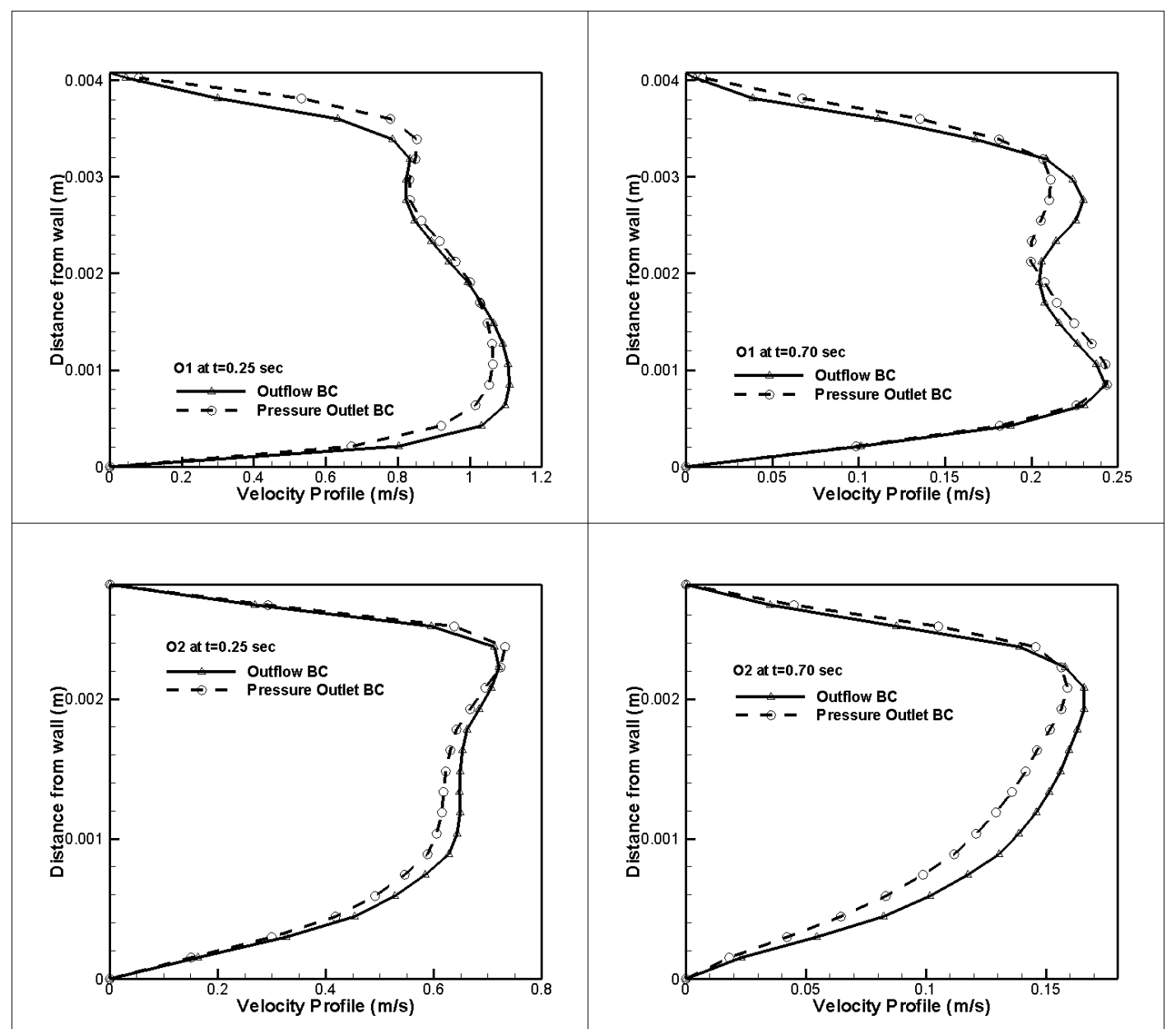

Figure 4.12: Velocity profiles at $\mathrm{O1}$ (top) and $\mathrm{O2}$ (bottom) cross sections at the systole peak (left) and late diastole (right) for Aneurysm-A using different boundary conditions

The highest difference between the velocities resolved using the pressure outlet $\mathrm{BC}$ and those resolved by the outflow $\mathrm{BC}$ could be as large as $8 \%$ for the $\mathrm{O} 1$ cross section and $10 \%$ for the $\mathrm{O} 2$ cross section during the end-diastole.

As explained above, the flow ratio imposed at the child arteries introduces a small error in the calculation of the velocity profiles of the outlets. This is why the differences at the systole peak are smaller compared to the late diastole. The maximum difference of the $\mathrm{O} 1$ and $\mathrm{O} 2$ cross sections are $4 \%$ and $7 \%$ respectively for the velocities resolved by either boundary condition. The consequence of such difference in velocity would affect 
the WSS calculation and must be considered before any conclusion is made concerning the role of WSS on the development of aneurysms.

Figure 4.13 shows the scalar concentration profiles at the predefined planes. During peak-systole (left of Figure 4.13) and end-diastole (right of Figure 4.13), the profiles using different types of BC at the outlets. At the BF cross section (Figure 4.13a), the profiles show a $15 \%$ difference in the scalar concentration values resolved by the pressure outlet and the outflow $\mathrm{BC}$ at both systole and diastole times.

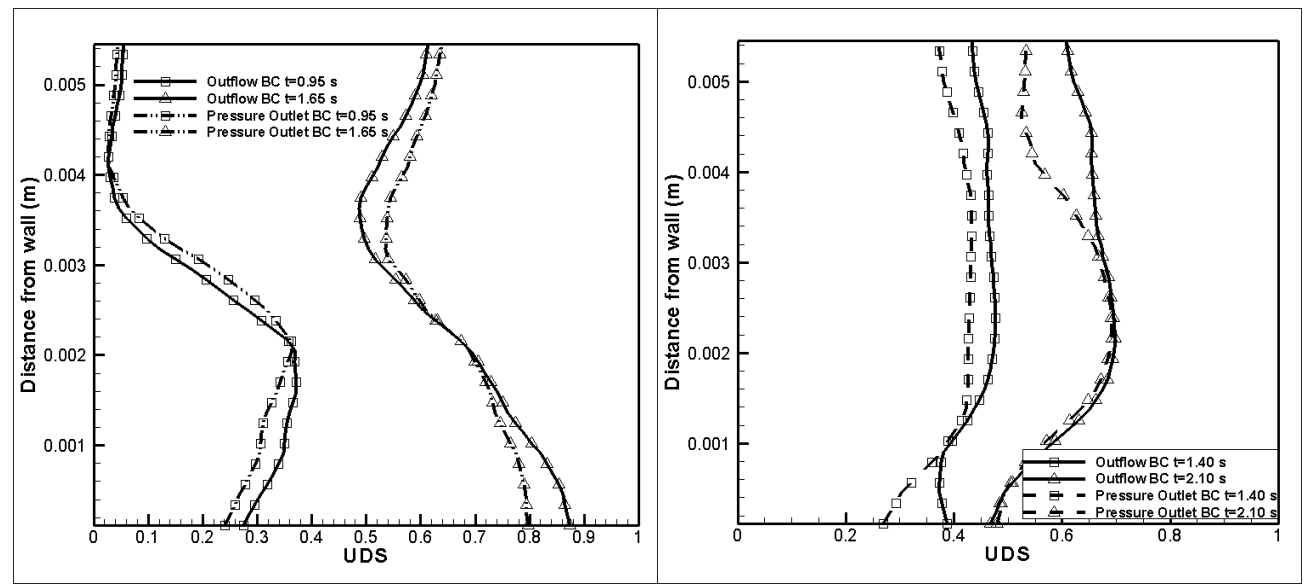

(a)
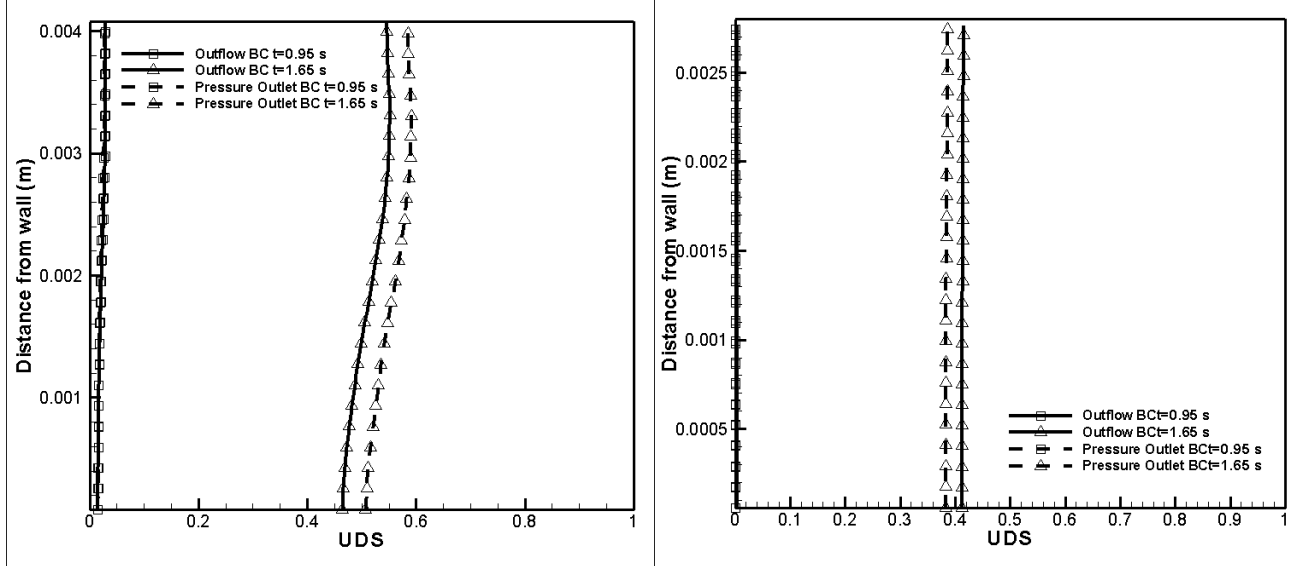

(b)

Figure 4.13: Scalar concentration in the Aneurysm-A using different boundary conditions at (a) BF cross section at systole (top left) and diastole (top right),(b) $\mathrm{O} 1$ at systole (bottom left), and $\mathrm{O} 2$ at systole (bottom right) 
For the outlet cross sections $\mathrm{O} 1$ and $\mathrm{O} 2$, Figure $4.13 \mathrm{~b}$ shows an approximate $10 \%$ difference in the scalar concentration values for both cardiac cycles (systole and diastole). Due to the proximity of the aneurysm to the outlets, the results may change close to the aneurysm region. To avoid these changes an extension of the outlets may be required. Further studies are needed to find out the required minimum distance of the outlet plane from the inlet of the aneurysm.

A healthy adult human has typical blood pressure values of $120 \mathrm{~mm}(16 \mathrm{kPa})$ and $80 \mathrm{~mm} \mathrm{Hg}(11 \mathrm{kPa})$ during the systolic and diastolic phases, respectively. The pressure values in the current study are scaled so that the diastole pressure is $80 \mathrm{~mm}$ of $\mathrm{Hg}$. Figure 4.14 shows the distribution of the total pressure along the Aneurysm-A geometry at the peak-systole $(\mathrm{t}=0.25)$. This relatively high pressure at the parental inlet must be higher compared to the outlets to overcome the viscous forces.

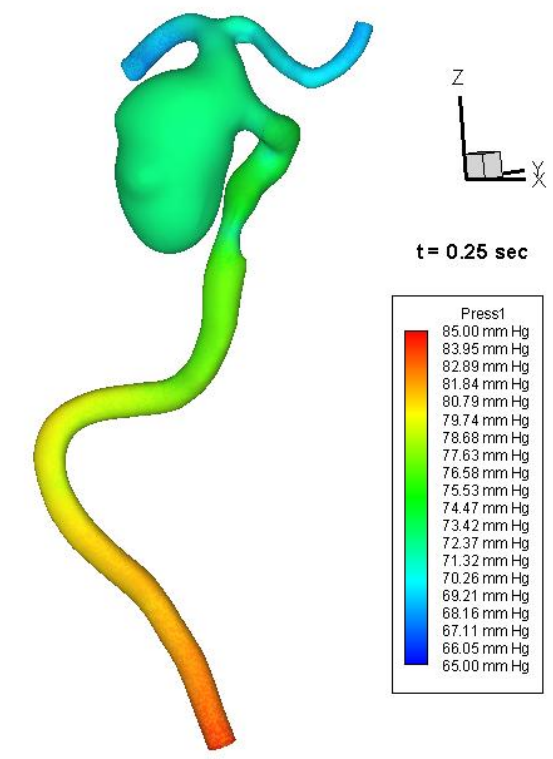

Figure 4.14: Total pressure at the peak of the systole $(t=0.25$ sec) for Aneurysm-A.

A summary of the total pressure results and the velocity magnitude are presented in Figure 4.15 during the cardiac cycle period. Figure 4.15 shows the velocity magnitude 
(top) and the static pressure (bottom) at the geometric center of the representative crosssections PI, EA, O1, and $\mathrm{O} 2$ as a function of time during one cycle. This figure also shows that the pressure and the velocity are nearly in phase, which agrees with the calculation of the dimensionless Womersley number. In the blood flow vessel's network, the parameters which influence the Womersley number, such as density, dynamic viscosity, and frequency remain the same along the parental artery, except for the artery diameter which shows very small changes. Thus, the Womersley number shows small changes, but remains in the interval $(1<\alpha<10)$.

Figure 4.15 may cause confusion, because when the velocity increases, the pressure should decrease according to Bernoulli's equation. However, in the current simulations the flow changes with respect to time (unsteady flow); thus, the flow is conserved and governed by continuity (Eqn. 4.3) and momentum (Eqn. 4.4) equations.

$\frac{\partial(\rho A)}{\partial t}=\frac{\partial \dot{m}}{\partial x}$

where $m=\rho u A$ and Navier-Stokes equation can be written as a function of the WSS

$$
\frac{\partial n \&}{\partial t}=-\frac{\partial}{\partial s}\left(u_{s} n \&\right)-A \frac{\partial P}{\partial s}-\tau_{w} \varsigma_{P}
$$



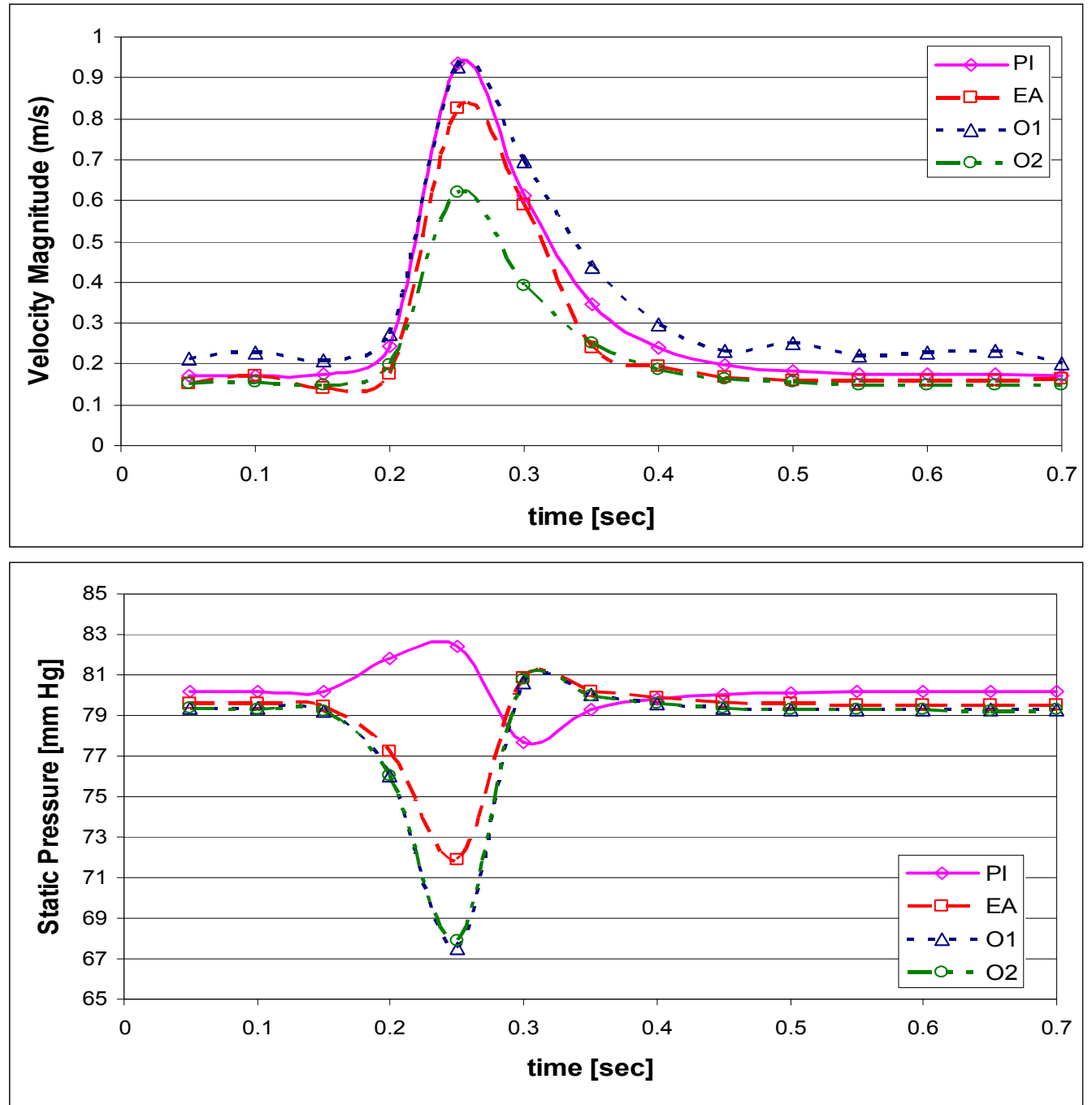

Figure 4.15: Velocity (top) and static pressure (bottom) waveform at the geometric center of the inlet cross section (PI) and representative cross-sections EA, $\mathrm{O} 1$ and $\mathrm{O2}$ during one cardiac cycle for Aneurysm-A 


\subsubsection{Discretization Scheme effects and Grid Dependence Study for Aneurysm-A}

The effect of the order of discretization scheme on the resolved velocity profiles is gauged by simulating hemodynamic flow through Aneurysm-A using first order and second order discretization schemes.

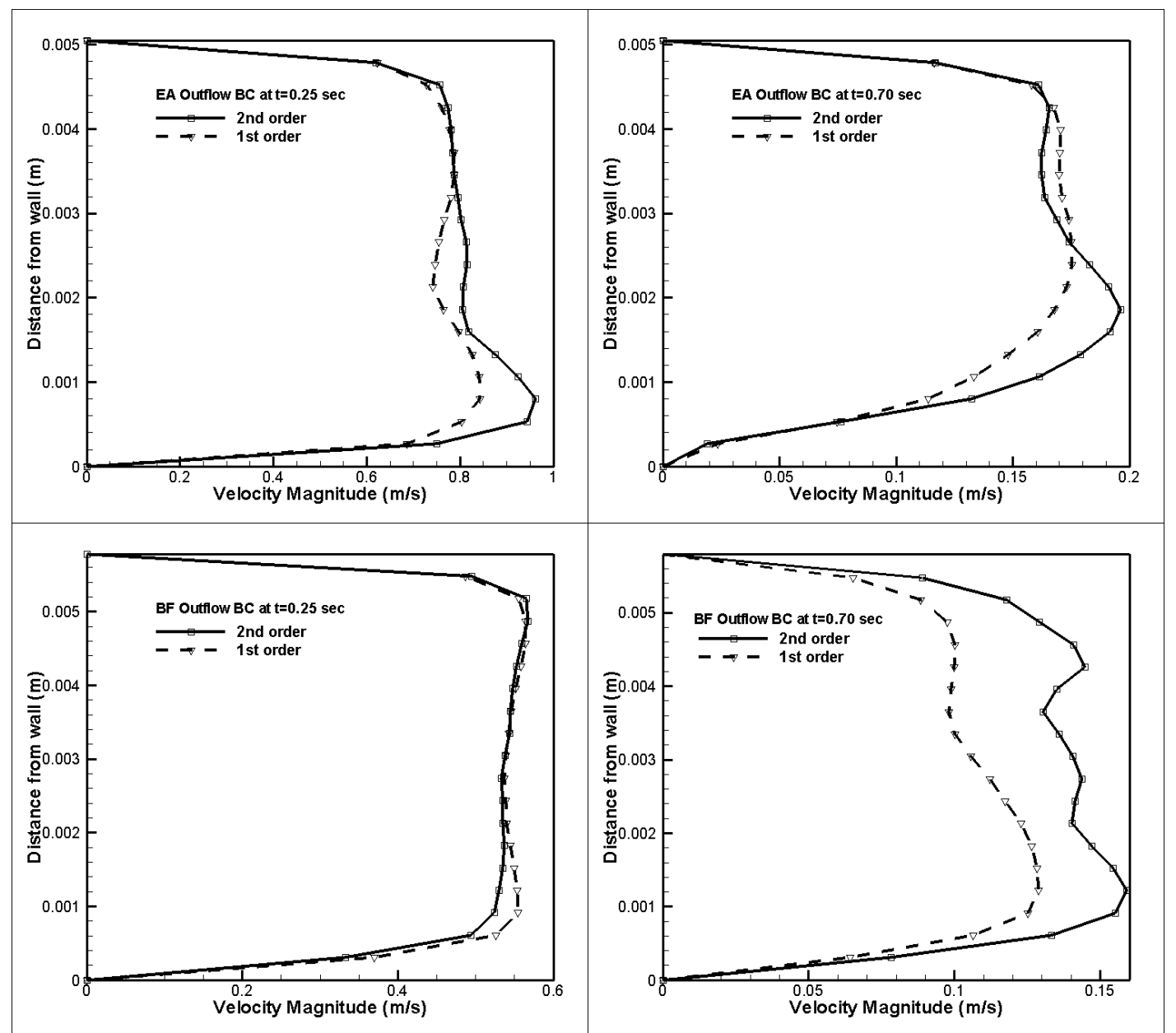

Figure 4.16: Velocity profiles at EA (top) and BF (bottom) cross sections at the systole peak (left) and late diastole (right) for Aneurysm-A using different discretization schemes 
Using a medium grid resolution (1.25 M cells, see Table 3.1) and an inlet pressure pulse similar to the ones used before, the simulations for different discretization schemes are carried out using an outflow $\mathrm{BC}$ and a mass flow ratio of $74.91 \%$ and $25.09 \%$ at the outlets $\mathrm{O} 1$ and $\mathrm{O} 2$ respectively.

The top of Figure 4.16 shows the velocity profiles using different discretization schemes at EA cross section. The maximum difference between these velocities profiles is less than $10 \%$ at the peak-systole (top left) and $18 \%$ at the late diastole (top right). Similar analysis is performed for the BF cross section as shown in the bottom of Figure 4.16. The velocity profiles at this plane show an estimated difference of $5 \%$ and $30 \%$ for the systole peak (bottom left) and the late diastole (bottom right) respectively.

Similar plots for the outlets cross sections are presented in Figure 4.17. Results show a maximum difference of $16 \%$ in the velocity profile at the systole peak (top left of Figure 4.17) and at the late diastole (top right of Figure 4.17) for $\mathrm{O} 1$ cross section. The differences in the velocity profiles at the $\mathrm{O} 2$ cross section are smaller at both phases of the cardiac cycle. At the peak of systole (bottom left of Figure 4.17), the estimate difference is about $12 \%$, while at late diastole (bottom right), the difference is approximately $6 \%$. When a second order scheme is used, the second order terms in the Taylor series expansion are retained. These terms, which are neglected in the first discretization scheme, characterizes details of the fluid flow better providing more accurate (circa 15\%) solutions. As mentioned, the truncation of the series greater than the first order also introduces errors in the volumetric flow ratios in both outlets less than $1 \%$. 


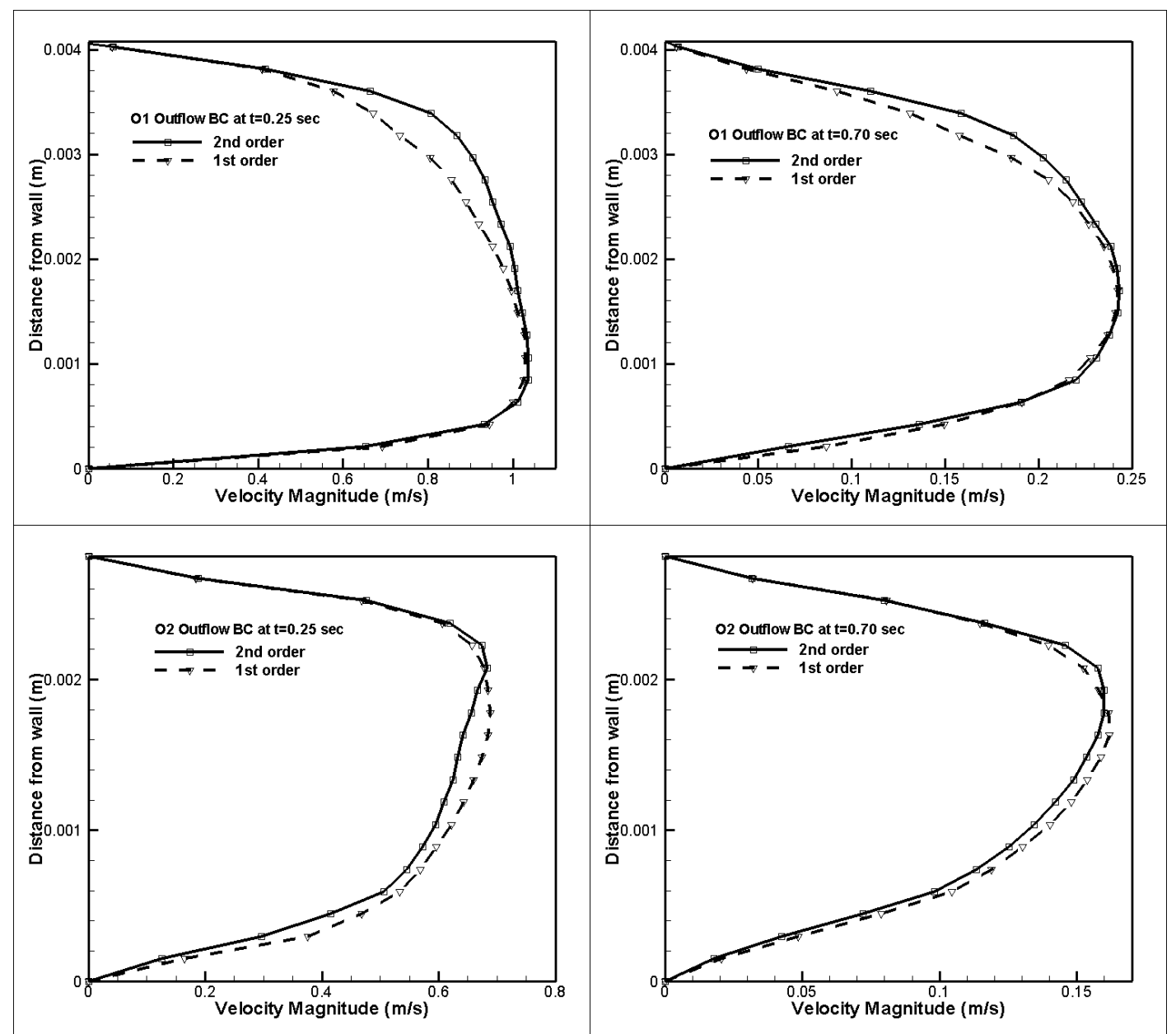

Figure 4.17: Velocity profiles at $\mathrm{O1}$ (top) and $\mathrm{O2}$ (bottom) cross sections at the systole peak (left) and late diastole (right) for Aneurysm-A using different discretization schemes

A grid convergence study is performed by using three different grid densities as shown in Table 3.1 to quantify the discretization error. This quantification error method suggested by ASME Journal of Fluids Engineering is widely accepted by the CFD community. This technique not only applies to velocities profiles, but also to the WSS profile along a defined line as shown in Figure 4.9. The line is traced along the region of interest to compare the WSS magnitude among the three grid resolutions. The WSS profiles are extracted on a line which passes around the aneurismal neck and the constriction region since this zone registered as the highest value. The reason why the 
location of the maximum WSS is included in the line extraction is because the highest WSS value is subject to elevated magnitude changes using finer grid resolution (Prakash et al, 2001). This variation is due to a relatively larger number of computational cells near the wall region, which are used to calculate the WSS more accurately.

The velocity profiles are compared for the three different grid resolutions at the four cross sections mentioned before. The GCI (Grid Convergence Index) method extrapolates to a profile nominally at zero grid size resolution, and the error bars correspond to the fine grid resolution.
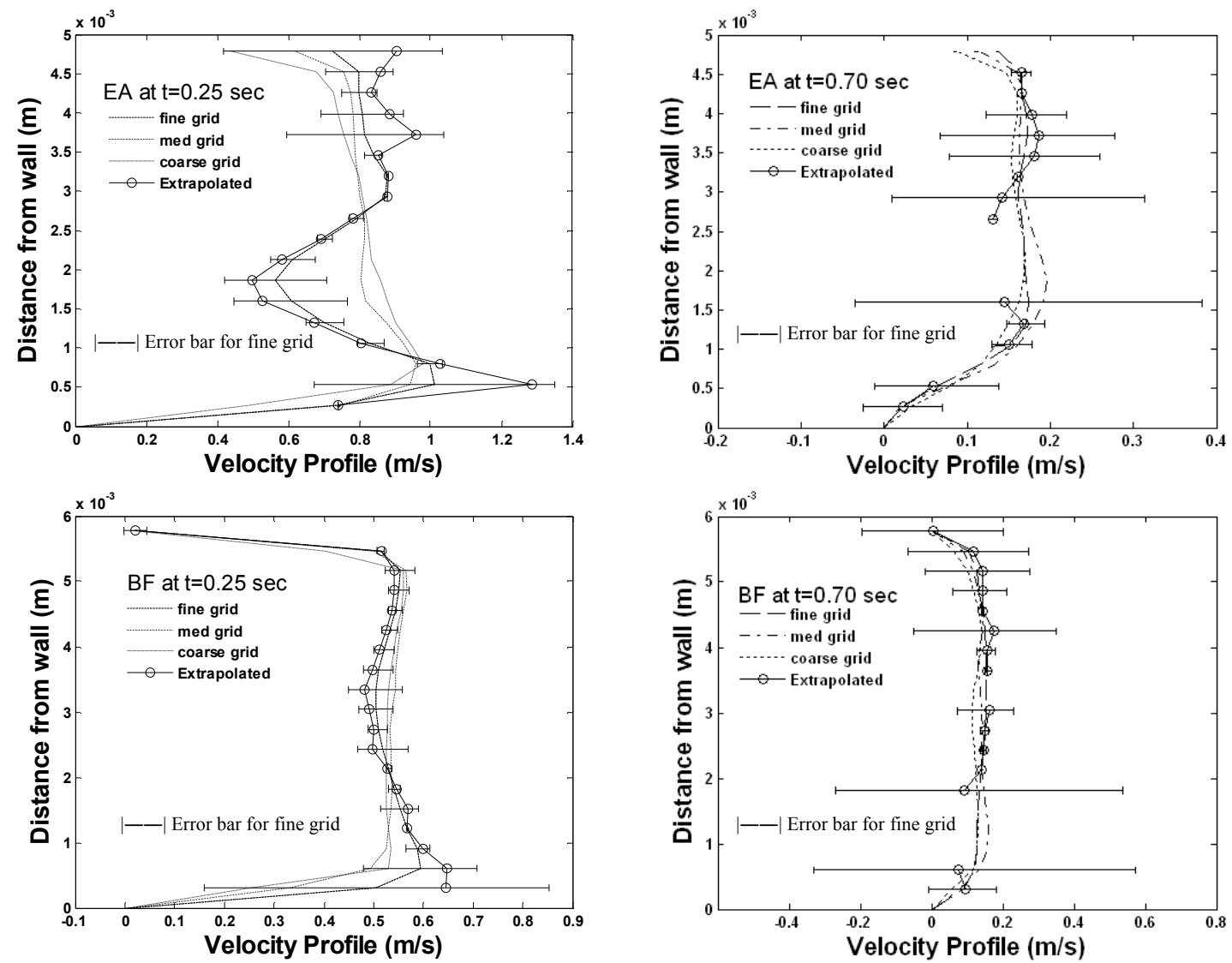

Figure 4.18: Velocity profiles at EA (top) and BF (bottom) cross sections at the systole peak (left) and late diastole (right) for Aneurysm-A using second order discretization scheme 
During the systole peak (left of Figure 4.18), the maximum approximate error between the fine and coarse grid is $30 \%$ and $41 \%$ on the cross sections EA and BF respectively. At the end-diastole (right of Figure 4.18), the maximum approximate error between the fine and coarse grid is $66 \%$ for both EA and BF cross sections. A two fold increase in the grid size leads approximately to $20 \%$ differences in the velocity profiles. The error bars in Figure 4.18 are calculated using the Richardson extrapolation in conjunction with the grid convergence index (GCI). Large error bars in the EA cross section at the systole peak and diastole (top of Figure 4.18) suggest the inadequacy of the finest grid in resolving the large velocity gradients found in this region. In the vicinity of the EA cross section, various dynamic phenomena such as flow entering the aneurysm, flow exiting the aneurysm, local acceleration/deceleration due to the curve in the artery leading up to the region and an overall pulsatile flow, all interact with each other to produce a highly complex flow field. The results and the error bars suggest that the simulations would benefit from a local grid refinement in this region. Similar behavior of large error bars at the BF cross section (bottom of Figure 4.18) is due to the difficulty in resolving velocity gradients due to the bifurcation of the geometry, incident and reflected flow in this particular region. The large error bars at the diastole-end may be a singular point where the error is very large due to almost zero difference $(\Delta \Phi)$ from grid to grid. 

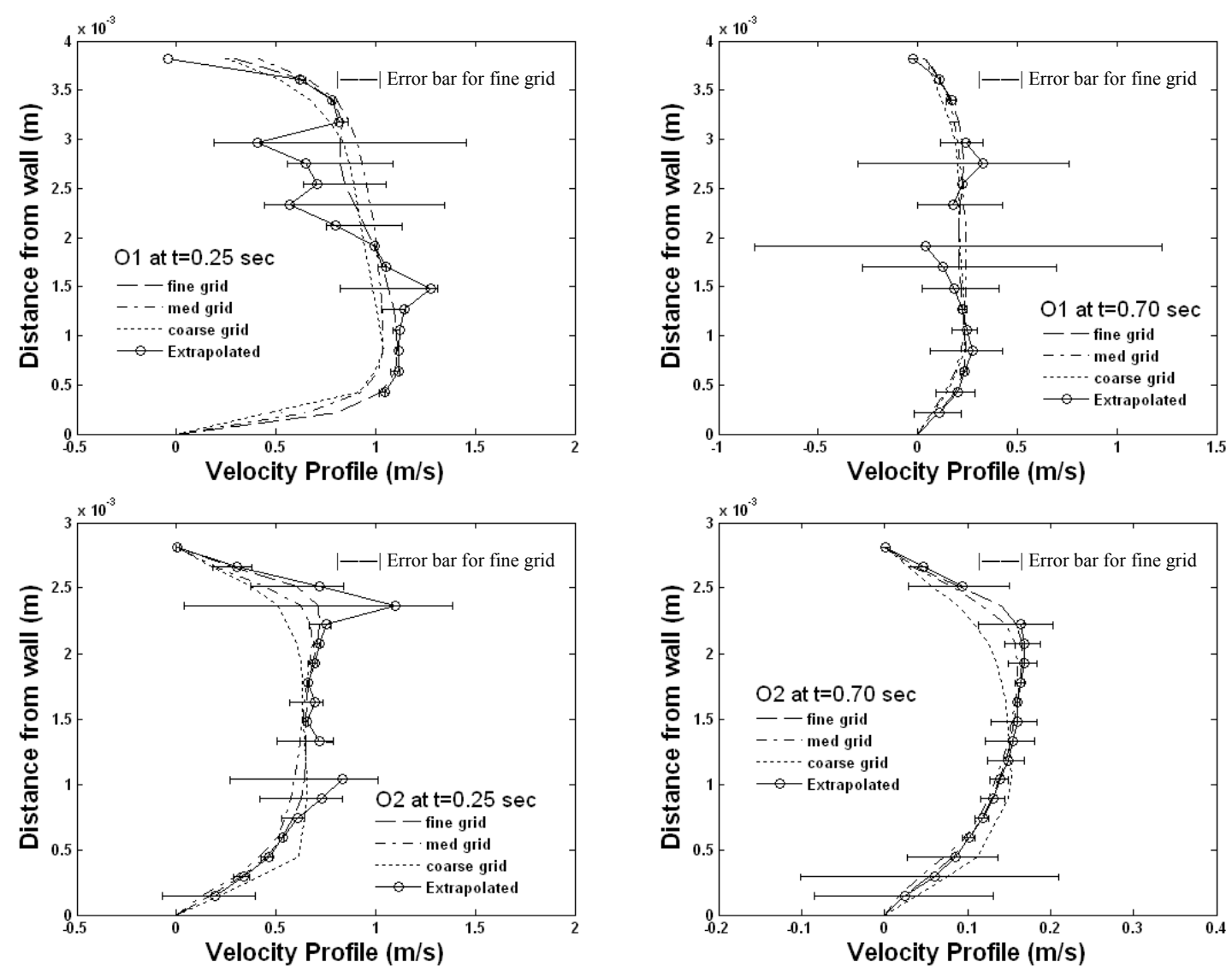

Figure 4.19: Velocity profiles at $\mathrm{O1}$ (top) and $\mathrm{O2}$ (bottom) cross sections at the systole peak (left) and late diastole (right) for Aneurysm-A using second order discretization scheme

In contrast to the EA and BF cross sections, the maximum approximated error between the fine and coarse grid is $35 \%$ and $47 \%$ at the outlets $\mathrm{O} 1$ and $\mathrm{O} 2$ respectively during the systole peak (left of Figure 4.19). While the maximum approximated error between the fine and medium grid is approximately $75 \%$ and $72 \%$ for the $\mathrm{O} 1$ and $\mathrm{O} 2$ outlets cross sections respectively, during the late diastole (right of Figure 4.19). The error bars in Figure 4.19 are calculated similarly as before using the RE (Richardson Extrapolation) and GCI method. Large error bars are seen in the $\mathrm{O} 1$ cross section at the systole peak and diastole (top of Figure 4.19) at the core region of the flow, while for the $\mathrm{O} 2$ cross section the large error bars are located mainly near to the wall region (bottom of 
Figure 4.19). These bars for the fine grid indicate some flow field complexity due to the curvature of the artery. Again the large error bars at the late diastole may be singular points where the error is very large due to almost zero $\Delta \Phi$ from grid to grid.

The most critical parameter, i.e. WSS is also analyzed using three different grid resolutions. The maximum WSS always occurs at the diameter artery constriction during the systolic peak, which is located in the upstream direction of Aneurysm-A, as seen in Figure 4.20. The highest magnitudes at this location are $13.01 \mathrm{~N} / \mathrm{m}^{2}, 18.83 \mathrm{~N} / \mathrm{m}^{2}$, and $24.68 \mathrm{~N} / \mathrm{m}^{2}$ for coarse, medium, and fine grid resolution, respectively. Similarly as Prakash (2001) found, this parameter demonstrated the most changes when the grid resolution is increased. Relative high shear stress values were also found at aneurysm's surroundings and at the other regions such as the artery bends. In addition, the fluid flow, which impacts obliquely at the aneurismal dome (upper region of the aneurysm), produces relatively high shear stresses while at the lower region of the aneurysm, low WSS are found. This lower aneurismal region is dominated by highly mobile and transient vortices as also shown in Figure 4.4. Therefore, it seems that the high WSS may be the cause of the aneurismal origin due to the curvature in the region of interest and the oscillatory transient vortices may further contribute to the weakening wall process. Further studies related to secondary flows are made in the next section comparing the hemodynamic results with and without the aneurysm in the region of interest.

Figure 4.21 shows the WSS profiles on a line traced along the surface of the aneurismal neck vessel (left) and along this line, three points are chosen to visualize the WSS change during a cardiac cycle (right). As mentioned, the traced line includes the stenosis region where the maximum WSS occur (named P1) and shows its changes 
during 0.7 seconds cycle. An increment of the grid resolution by a factor of approximately three, the highest WSS magnitude changed $70 \%$, while the other regions have an average growth of $38 \%$ approximately at the systole peak. Hence, this shows a relative inaccuracy of the WSS calculation. Like the velocity profile extrapolation of the fine grid, the WSS is extrapolated for zero grid resolution. Along the traced line profile, points $\mathrm{P} 2$ and $\mathrm{P} 3$ are also chosen to correspond to an upstream and downstream point to the aneurysm throat. The WSS magnitudes of these points show relatively high values at systole and their variation during the cardiac cycle is shown at the right side of Figure 4.21. It is noted that oscillatory WSS between $10 \mathrm{~N} / \mathrm{m}^{2}$ and $3 \mathrm{~N} / \mathrm{m}^{2}$ is exhibited these points (P2 and P3) which may lead to a gradual weakening of the arterial wall, modifying its structure. This aneurysm was treated by coiling the dome of the aneurysm, but the author does not know how effective the treatment was. 


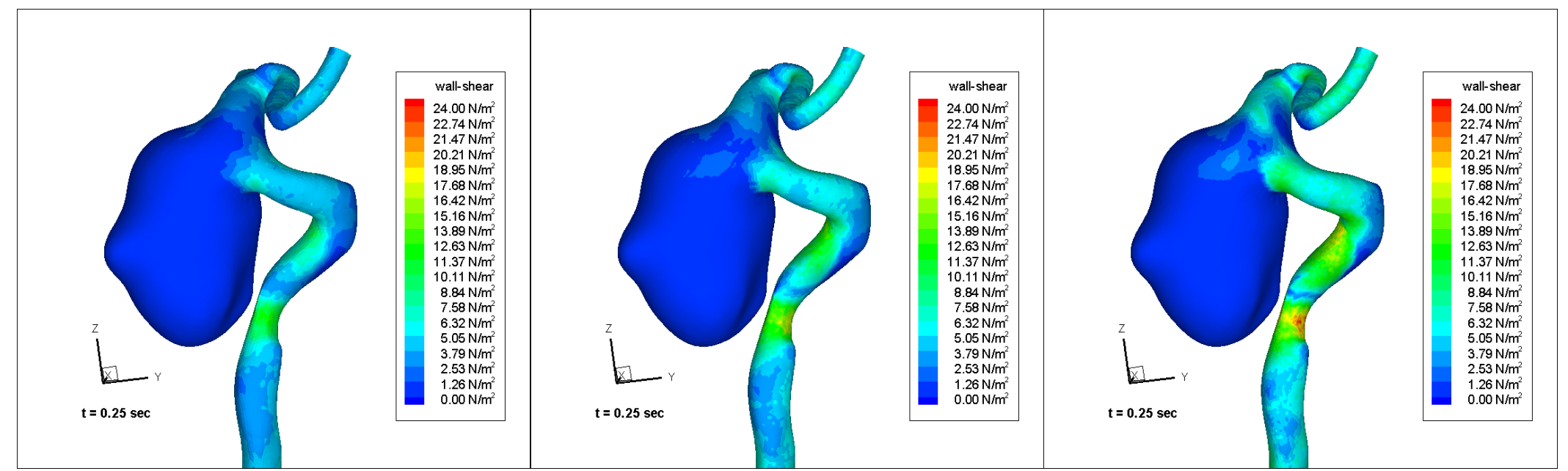

Figure 4.20: Wall shear stress contours for Aneurysm-A using different grid resolution at the systole peak: coarse, medium, and fine grid resolution.
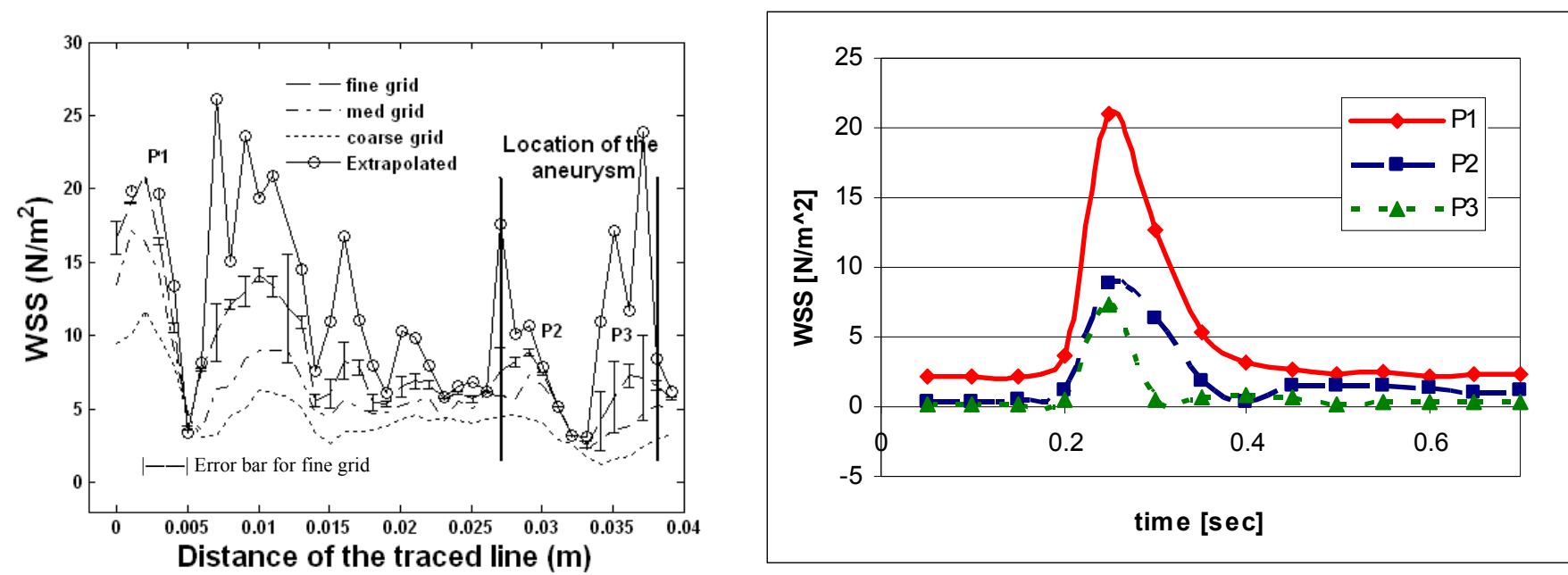

Figure 4.21: Results of the WSS along the traced line at the systole peak (left) and oscillatory WSS during a cardiac cycle at three defined points (right) for Aneurysm-A 


\subsubsection{Results for Geometry-A without the aneurysm}

The removal of the aneurysm is performed to investigate the hemodynamic causes which create the aneurysm in the first place. A grid dependence study is also performed using only a second order discretization scheme. In the previous section, the use of second order term in the Taylor series expansion was shown to achieve more accurate results. New cross sections (as shown in Figure 4.9) are defined in the region of interest to study the flow characteristics. This approach will provide an estimate of the hemodynamic factors which initiate the aneurysm occurrence when the artery wall is healthy.

At these cross sections, the discretization error is also estimated for three different grid sizes using CGI methodology. Figure 4.22 shows the velocity profiles at three locations on the geometry-A (without aneurysm). During the peak-systole (left of Figure 4.22), the maximum difference between the fine and the medium grid velocity profiles is $9 \%$ for BA cross section, $16 \%$ for IS cross section, and 33\% for the AA cross section. On the other hand, the approximate difference between the velocity profiles on the fine grid and the medium grid are recorded as $12 \%$ for the BA cross section, $3 \%$ for the IS cross section, and $5 \%$ for the AA cross section at the late diastole (right of Figure 4.22). Some error bars of the fine grid at the end-diastole are not considered in the calculation of singular inflection points. 

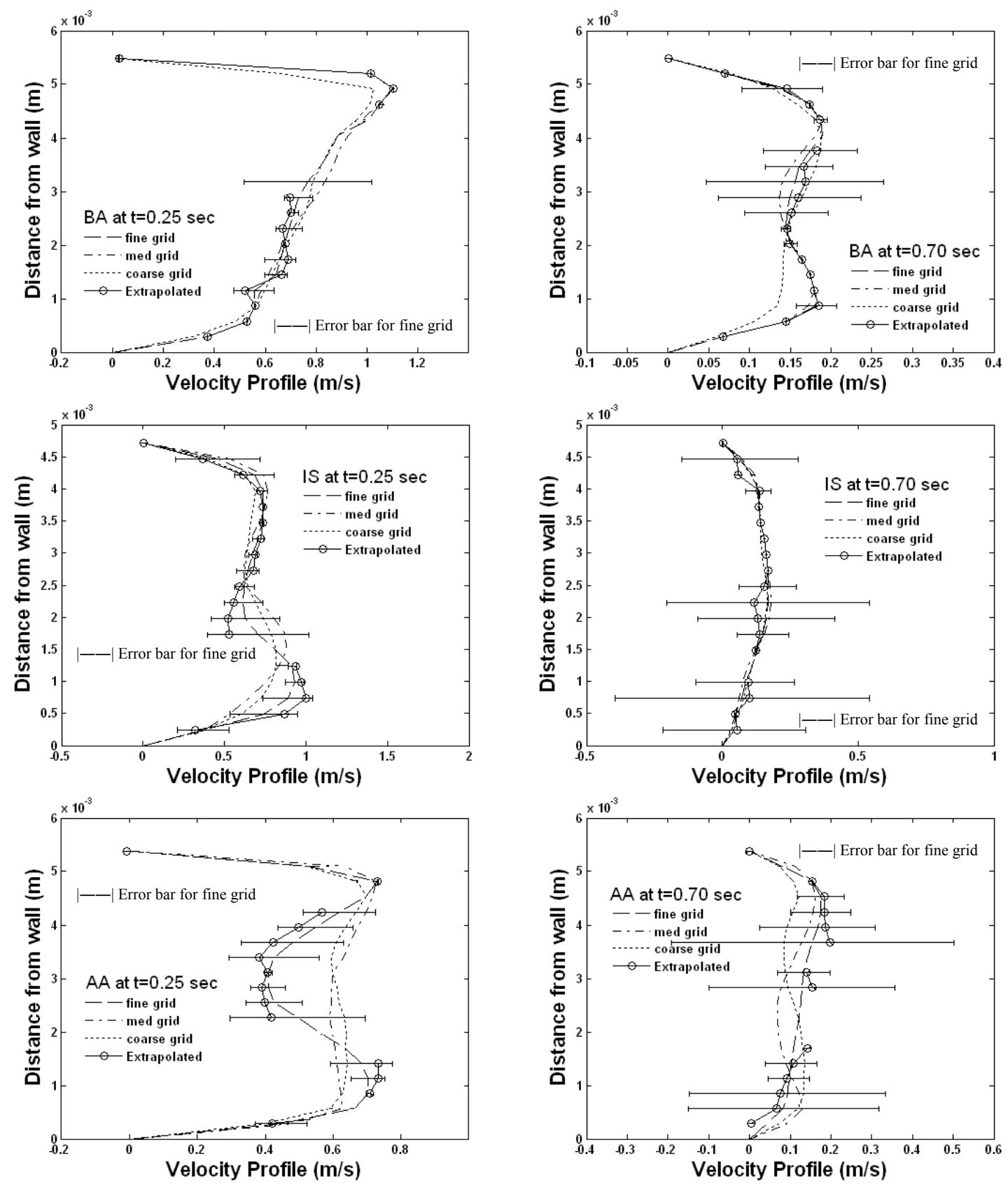

Figure 4.22: Velocity profiles at different locations of the geometry without the aneurysm at the systole peak (left) and the late diastole (right): BA (top), IS (middle), and AA (bottom)

It is also noted that the removal of the aneurysm eliminates the dynamic flow pattern entering and exiting the aneurysm, leading to more stable flow patterns. The new patterns for this geometry are only affected by the acceleration/deceleration velocities (pulsatile 
flow), the curvature of the arterial geometry, and the artery bifurcation. During the acceleration phase of the pressure pulse, the flow is resolved with less error (left part of Figure 4.22), while in the deceleration or stationary part of the pressure pulse, the calculation of the flow velocity includes higher discretization errors. Hence, a finer grid or smaller time step might be required to resolve the dynamics of the decelerating phase of the pulsatile flow. Additionally, a small error is introduced at the arterial bifurcation flow rate during the outflow $\mathrm{BC}$ because it is imposed on the volumetric rate found at the systole peak of the pressure outlet BC. This change would have some effect in the resolve velocities due to the flow wave reflected in the upstream direction. Finally, special attention must also be paid to the pressure solver and the $\mathrm{BC}$ for the pressure terms along with the mesh requirement to obtain a truly mesh independent solution.

Even though the location of the maximum WSS (stenosis region) remains invariable using different grid resolutions, the current investigation would focus on this region of interest. These maximum WSS values are $13.7 \mathrm{~N} / \mathrm{m}^{2}, 25.46 \mathrm{~N} / \mathrm{m}^{2}$, and 25.63 $\mathrm{N} / \mathrm{m}^{2}$ for coarse, medium, and fine grid resolution, respectively, as shown in Figure 4.23. At the region of interest where the aneurysm was initially located, relatively high WSS can be observed due to the arterial curvature. When the grid resolution is increased by a factor of three, the changes of the calculated WSS are less. This change does not necessarily mean that the grid resolution is suitable for WSS calculations. This parameter is extremely sensitive to the grid size and hence it can only be validated through experiments. Moreover, a line traced on the region of interest in combination of GCI method would provide an estimation of how accurate the current results are. This new line extraction of the WSS readings crosses the region where the aneurysm was originally 
located and includes the maximum WSS area (see Figure 4.9). The time responses of the WSS at selected locations on the extracted line are also presented in Figure 4.24 (left side). Likewise, Point 1 (P1) corresponds to the location of the stenosis region where the maximum WSS of the geometry-A occurs. P1 encompass a cyclic variation in a grid dependence study as is supported by Prakash (Prakash, 2001). Other points, P2 and P3, are located in different positions than the ones situated in the aneurysm-A geometry. These points belong to the region of the artery wall reconstruction, which the WSS magnitude changes about $40 \%$ with each grid refinement. This percentual change will decrease for every grid refinement. Figure 4.24 (left side) also shows the extrapolated value for a zero grid size for the three points being the highest magnitude the one which changes the most. According to the extrapolated value on the region of interest, the WSS would oscillate between $18 \mathrm{~N} / \mathrm{m}^{2}$ and $9 \mathrm{~N} / \mathrm{m}^{2}$ at the systolic peak. Comparison of these values with the computational WSS magnitudes $\left(8 \mathrm{~N} / \mathrm{m}^{2}\right)$ for the fine grid resolution on the reconstructed region show a difference averaged of $50 \%$ approximately. Thus, the calculated WSS is not accurate enough to claim accurate results, leading to premature conclusions. The right side of Figure 4.24 show the cyclical changes of these three points during the cardiac cycle. The time response of WSS for P2 and P3 show a change from very low values to $11 \mathrm{~N} / \mathrm{m}^{2}$ and $8 \mathrm{~N} / \mathrm{m}^{2}$ respectively between the systole and the diastole. Hence, the cyclic stress reversal on the artery wall may lead to degeneration of the wall material leading to the initiation of an aneurysm, as stated before for the geometry with the aneurysm. Oscillatory WSS also occurs at other arterial curvatures of the geometry which may be potential zones for aneurismal formation. 


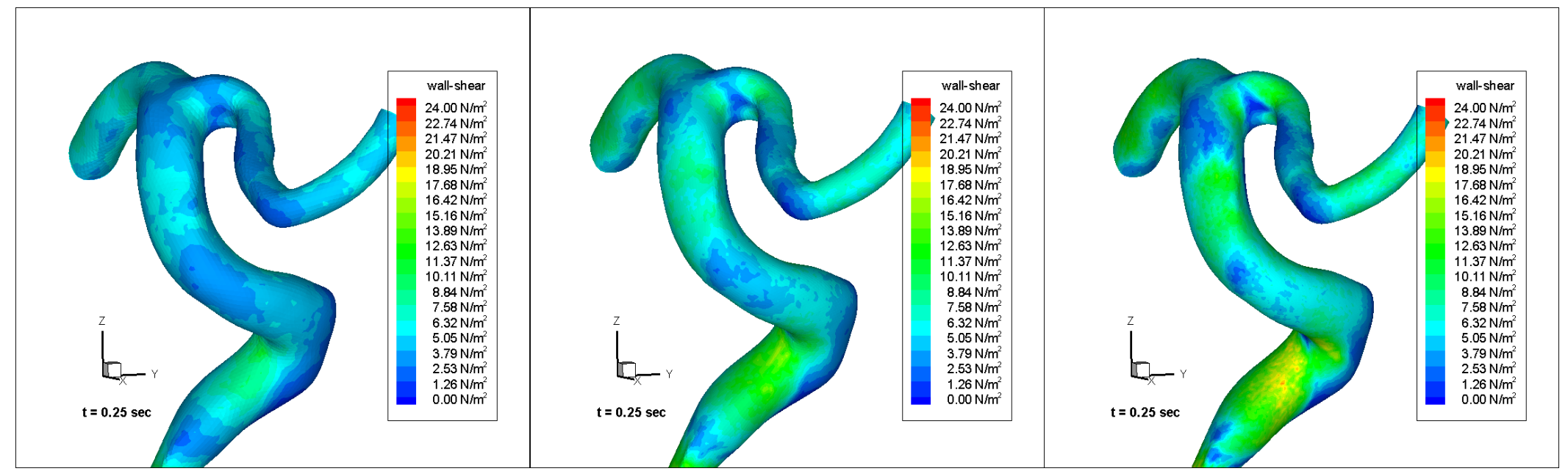

Figure 4.23: Wall shear stress contours for geometry-A using different grid resolution at the systole peak: coarse, medium, and fine grid resolution
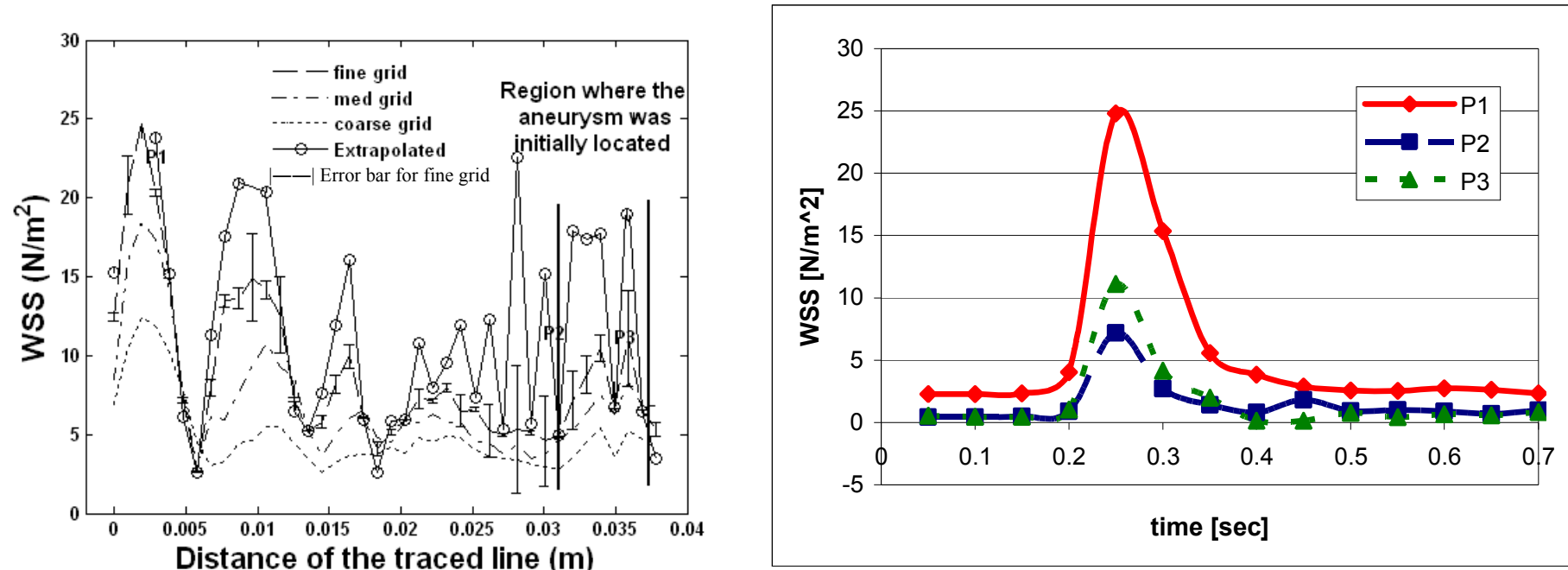

Figure 4.24: Results of the WSS along the traced line at the systole peak (left) and oscillatory WSS during a cardiac cycle at three defined points (right) for geometry $A$ 
In order to investigate the other hemodynamic factors that may cause intracranial aneurysms, the flow characteristics are investigated at the same three cross sections as before. These cross sections are also analyzed in the Aneurysm-A geometry for comparison purposes. At the BA cross section, no major difference is found during the cardiac cycle. However, at the other cross sections (IS and AA), the flow behaves differently due to the aneurysm. The changes are mainly observed from the systole peak to the latter recorded times (deceleration process). To display the different flow fields at these cross sections, cardinal directions are used as references. Same scales are used for the velocity vectors to facilitate the discussion.

Figure 4.25 shows the velocity vectors colored by the dynamic pressure for the systole peak and the beginning of the deceleration phase at the IS cross section. The images on the top correspond to the geometry with the aneurysm, while the ones on the bottom correspond to the geometry without the aneurysm. At the IS cross sections with the aneurysm, the fluid flow comes from the upstream direction through the artery and it rotates in a clockwise direction impinging at the north western region of the artery wall during the systole peak. When the deceleration phase starts, the flow becomes more complex appearing as secondary flows. Three vortical recirculation zones are observed at $\mathrm{t}=0.30$ seconds, one located at the north region with clockwise direction, at which it starts dissipating. The other two swirling eddies, located at the south region, collapse due to the opposite directions of the vortices creating a weak vortex which is dissipated at $\mathrm{t}=0.35$ sec. On the other hand, the bottom of Figure 4.25 shows the flow characteristics at the same cross sections at the same instant as before. At the systole peak, the flow impinges on the north west region of the vessel wall, splitting the flow in two resolved secondary 
vortices. Due to the location where the flow division occurs, the right vortex rises progressively in magnitude compared to the left vortex. Hence, the right vortex rotates in the clockwise direction, impinging in the entire northern region extending until the south east region. This extension of the rotational flow generates the formation of another vortex placed at the south west region. During the deceleration phase of the cardiac cycle $(\mathrm{t}=0.30 \mathrm{sec})$, these two vortices merge in a stronger clockwise vortex situated at the north east region. At the late systole $(\mathrm{t}=0.35 \mathrm{sec})$, the recirculation zone located at the north east region is pushed toward the west region, conserving the flow direction. At the same time, two other weak recirculation zones are formed at the north western and south west region of the IS cross section. The south west spinning flow exhibits a counter-clockwise direction, an opposite trend compared to the previous instantaneous time of the south east region vortex. Opposite flow directions are also found at the west region where the aneurysm happened. The vortex rotates in the counter clockwise path at the systole peak, while the vortex whirls in the clockwise direction at the late systole. This change of direction in these critical areas, the flow impingement due to the curvature of the artery (generating the WSS on the lumen vessel), and the flow wave reflected caused by the arterial bifurcation may aggravate the structural properties of the endothelial cells.

In addition, oscillatory vortices with opposite directions are observed in certain regions of the aneurismal dome as shown in Figure 4.7. Even though the WSS is very low, the arterial wall will grow continuously in size until it breaks. This may be possible once the endothelial cells of this region are debilitated. The force exerted by the oscillatory changing vortex direction would be the reason to rupture the wall. Although this hypothesis cannot be confirmed, there is also a lack of information of diseased artery 
wall properties. Further studies are needed to be investigated the structural properties of the ill aneurismal lumens.

Some other regions, such as the curvature located in the upstream direction of the region of interest are also affected by the flow impingement, generating a relatively high WSS. Recirculation vortices with opposite directions may happen during the deceleration phase; however the flow wave reflected due to the bifurcation region would play a decisive role triggering cerebral aneurysms. The diameter of this curvature region is calculated through approximated circular rings for various segments and the results show a greater diameter in the steep zone as it can be observed in Figure 4.23. This means that these are also potential regions where aneurysms may start to form.

Similar to the IS cross section, velocity vectors colored by the dynamic pressure at AA cross section are created to study the flow dynamics (Figure 4.26). During the cardiac cycle, it is found that major changes occur during the systole peak and in the deceleration phase for both geometries (with and without the aneurysm). The flow dynamics had been modified due to the aneurysm where the primary flow enters and exits from the dome toward to the outlets. When the deceleration phase starts, two swirl flows are formed at the south (strong vortex) and north region of the AA cross section at the top. At the later instantaneous recorded time, three vortices are formed at the west, north, and east region of the cross section. 


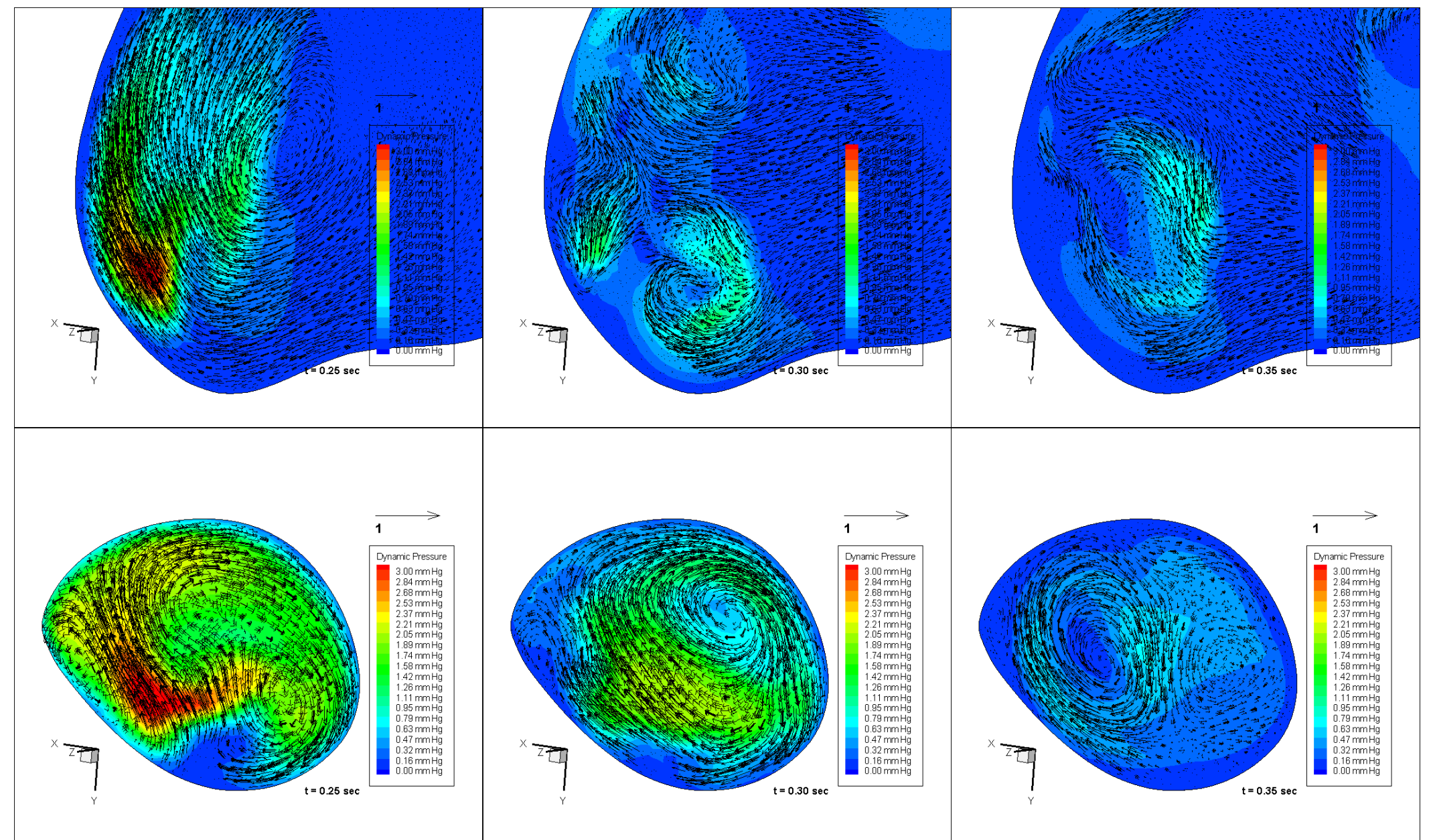

Figure 4.25: Velocity vectors colored by the dynamic pressure contours (mm Hg) for IS cross section with the aneurysm (top) and without the aneurysm (bottom) 
On the other hand, the geometry without the aneurysm (bottom of Figure 4.26) shows only one swirling flow region in the north region at the same cross section. This vortical flow spins in the clockwise direction and it starts dissipating energy due to the viscous forces in latter times of the cardiac cycle peak. Hence, the resolve flow characteristics are more dynamically complex due to the curvature and the aneurismal dome compared to the flow characteristics of the geometry without the aneurysm.

It is not surprising that the geometric configuration affects strongly the flow dynamics in geometries. The influence of the aneurismal curvatures found in the current investigation are potential regions to the origin of aneurysms which is supported by many researchers (Feng et al, 2004; Foutrakis et al, 1999; Gonzales et al, 1992, Kim et al 1992a; Kim et al, 1992b). Due to the flow unsteadiness, directional change of the secondary flows in the radial direction of the artery may degenerate the internal wall region of the vessels. The directional change of the vortical flows can also occur in the streamwise direction as a consequence of the reflected flow of the arterial bifurcation close to curved artery. The combination of these characteristics may lead to the formation of cerebral aneurysms due to the repeatedly weakening process of the endothelial cells during many years. 


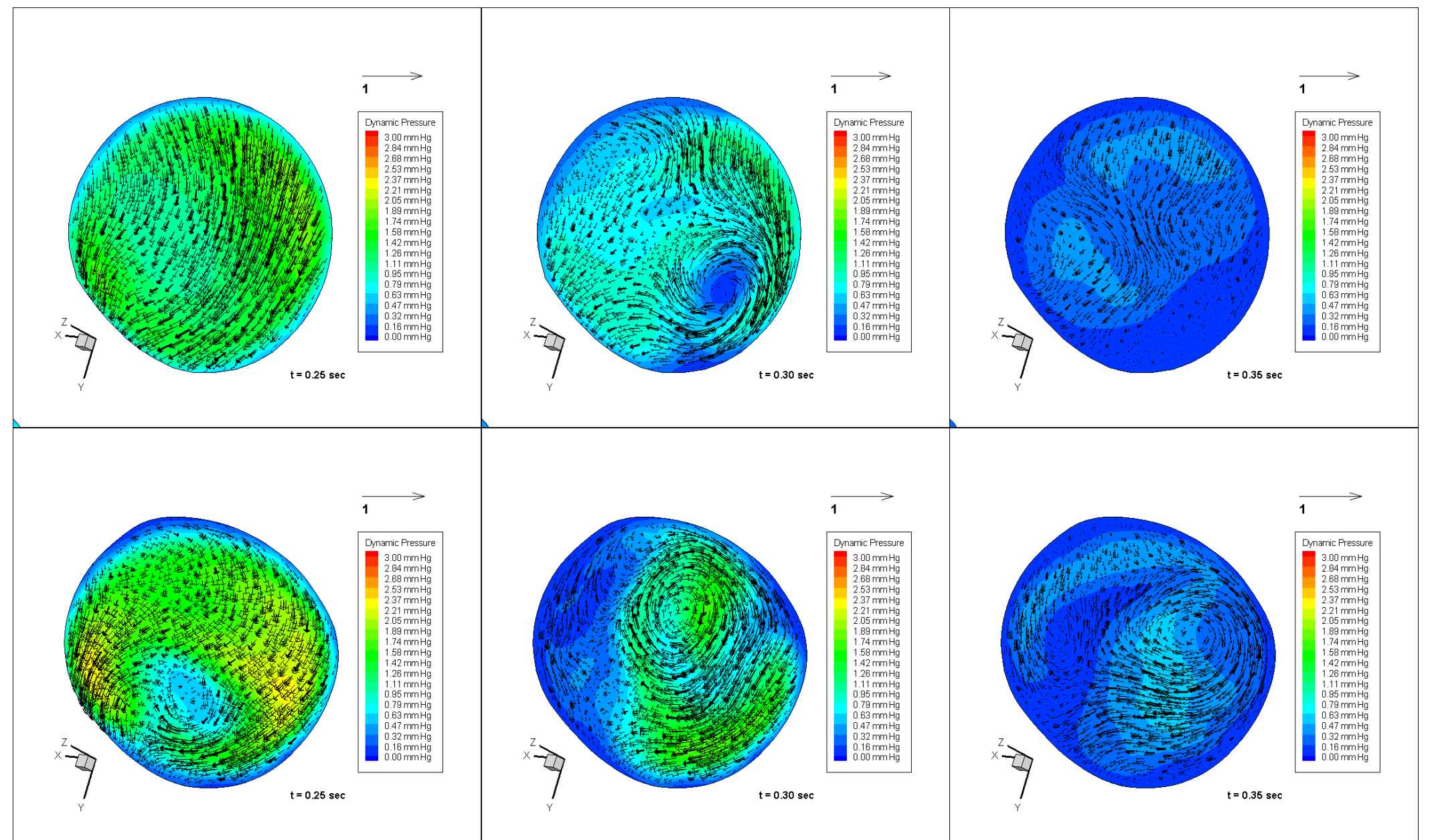

Figure 4.26: Velocity vectors colored by the dynamic pressure contours ( $\mathrm{mm} \mathrm{Hg}$ ) for AA cross section with the aneurysm (top) and without the aneurysm (bottom) 


\subsubsection{Results for Aneurysm-B}

\subsubsection{Boundary Conditions for Aneurysm-B}

FLUENT simulations were carried out for Aneurysm-B geometry using similar computational parameters as Aneurysm-A. The velocity profiles were generated by using two different $\mathrm{BC}$ and the results are compared on representative cross-sections. The volumetric flow profile used at the inlet for aneurysm-B was similar to the one used for aneurysm-A (see Figure 3.4) and the results are compared at the same peak-systole $(\mathrm{t}=$ $0.25 \mathrm{sec})$ and end-diastole $(\mathrm{t}=0.70 \mathrm{sec})$. Using pressure outlet $\mathrm{BC}$, the volumetric flow rates out of the outlets $\mathrm{O} 1$ and $\mathrm{O} 2$ were found to be $87.58 \%$ and $12.42 \%$ at the systole and $89.36 \%$ and $10.64 \%$ at diastole. The volumetric flow rate found using pressure outlet BCs is imposed in a new simulation using outflow BC at the dual outlets.

Similar to aneurysm-A, the impedance is calculated for the PI, O1, and $\mathrm{O} 2$ cross sections using different $\mathrm{BC}$ as shown in Figure 4.27. The magnitude of impedance in hemodynamics describes not only the amplitude of the total pressure and volumetric flow rate, but also the relative phase. Figure 4.27 shows that the impedances at PI, O1 and O2 cross sections exhibits similar shape and magnitude using different $\mathrm{BC}$. This means that flow resistance is very similar for the current geometry and the positive magnitude of the impedance for the cardiac cycle acts as a pressure sink at the outlets. 


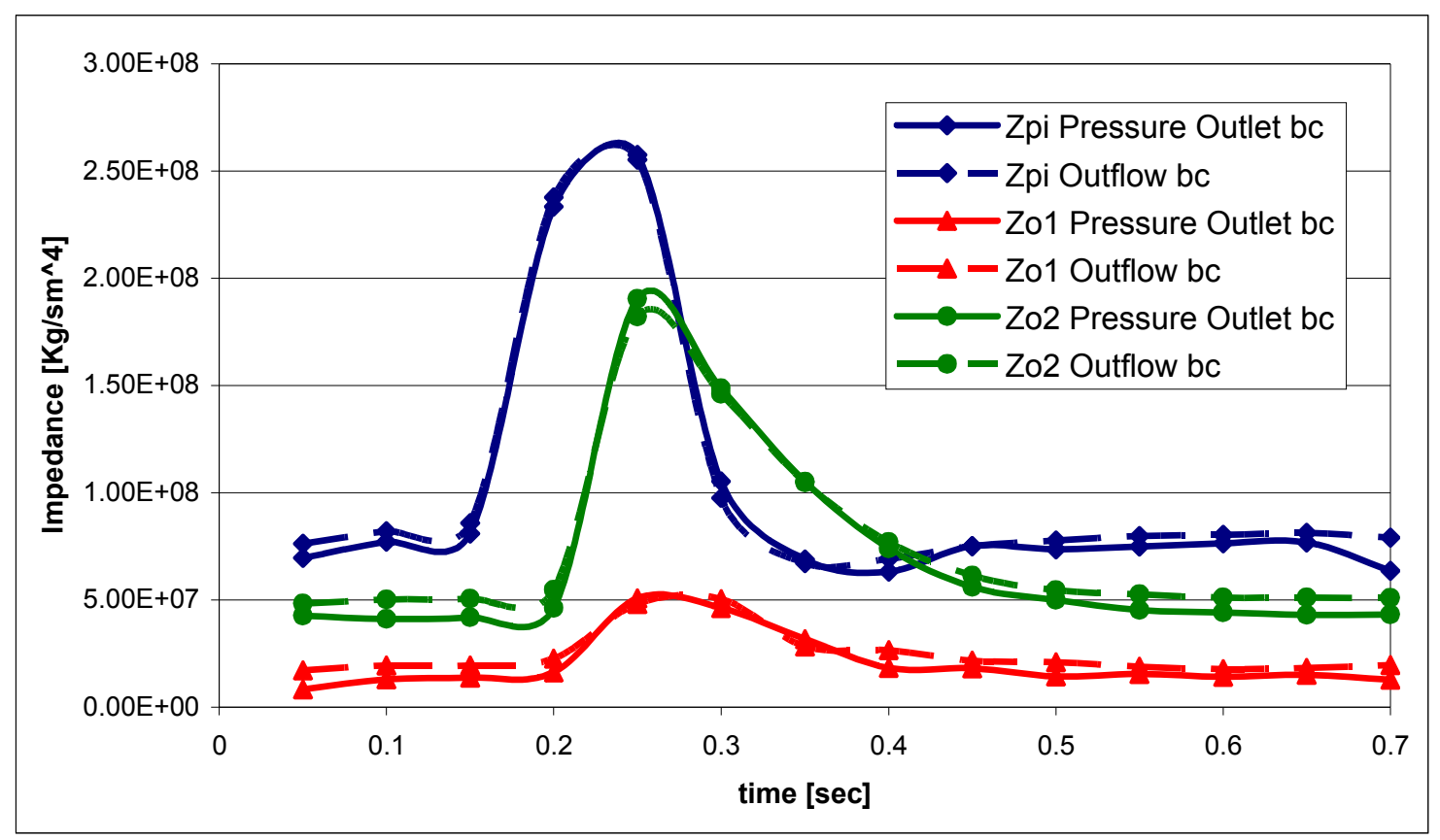

Figure 4.27: Impedance comparison between Pressure Outlet BC and Outflow BC at various locations during a cardiac cycle for Aneurysm-B

Comparisons of the different velocities' profiles at the defined cross sections are performed to calculate their difference. Figure 4.28 shows the velocity profiles using different BC for cross sections EA (top of Figure 4.28), and BF (bottom of Figure 4.28) analyzed in the systole peak (left) and late diastole (right). There is no difference in the velocity profiles for EA and BF cross sections at the peak-systole, while small differences are observed at the end-diastole, the maximum of $8 \%$ and $9 \%$ corresponding to EA and BF cross section respectively. These small changes at the end-diastole are due to the systolic volumetric flow rate obtained using pressure outlet $\mathrm{BC}$, which is imposed at the outlets $(87.58 \%$ and $12.42 \%$ for $\mathrm{O} 1$ and $\mathrm{O} 2$ respectively). A comparison of the scalar profiles shows no major differences at the EA and BF cross sections as it is shown in Figure 4.30. As mentioned before, further studies are required to calculate the minimum distance of the outlet planes so the BC would not have any effect on the solution. 


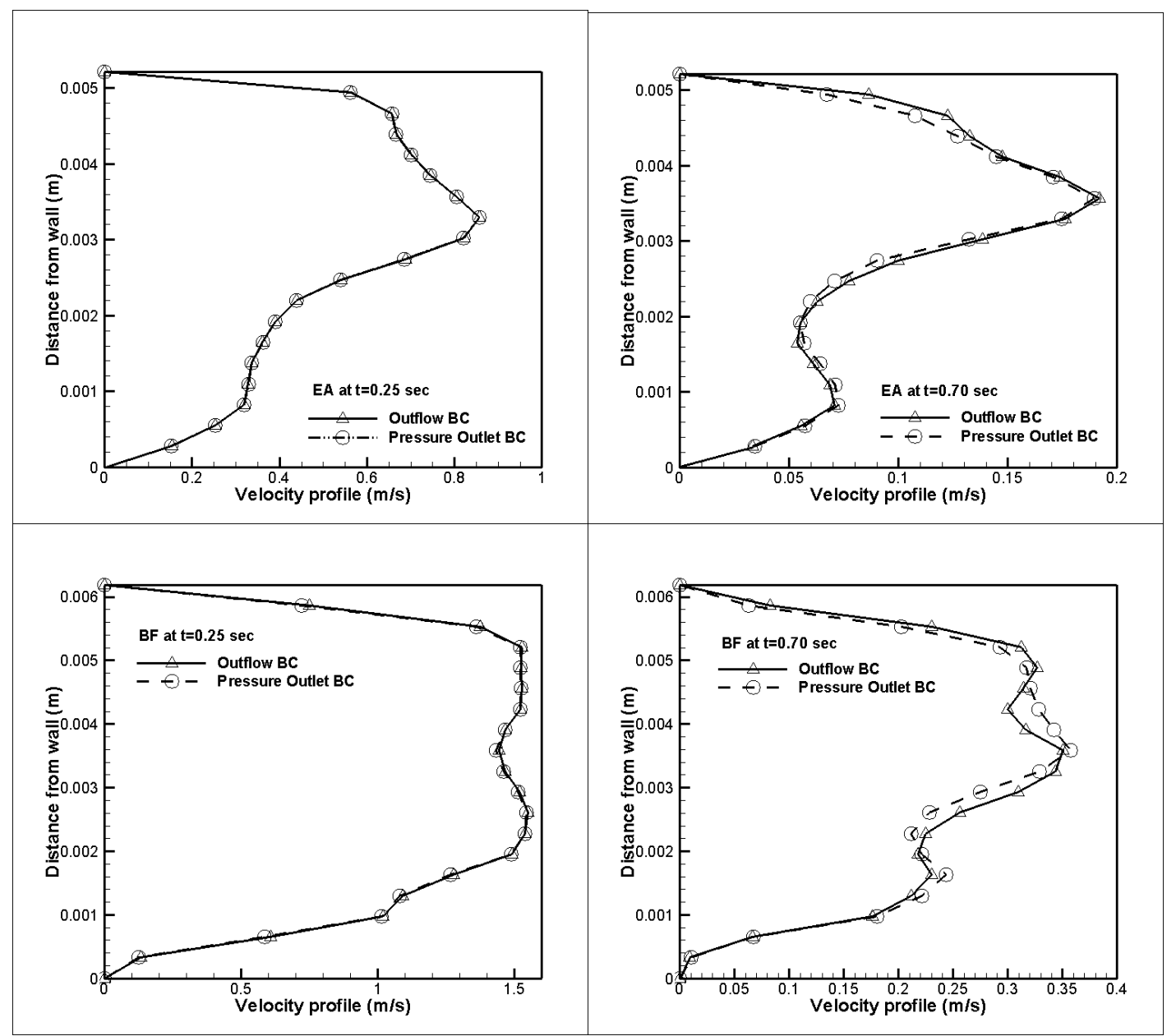

Figure 4.28: Velocity profiles at different locations for Aneurysm-B: EA at systole (top left) and diastole (top right), BF at systole (bottom left) and diastole (bottom right) using different boundary condition

Figure 4.29 shows the velocity profiles using different $\mathrm{BC}$ at the outlets' cross sections at the systole peak (left) and late diastole (right). The images on the top of Figure 4.29 correspond to $\mathrm{O} 1$ cross section and the images on the bottom of the Figure 4.29 correspond to the $\mathrm{O} 2$ cross section. The maximum differences in the velocity profile at O1 cross section is $20 \%$ for the peak-systole and $15 \%$ for the end-diastole, while on the small outlet cross-section $(\mathrm{O} 2)$, the velocity profiles show a difference as high as $12 \%$ at systole peak and of $22 \%$ at late diastole respectively. 


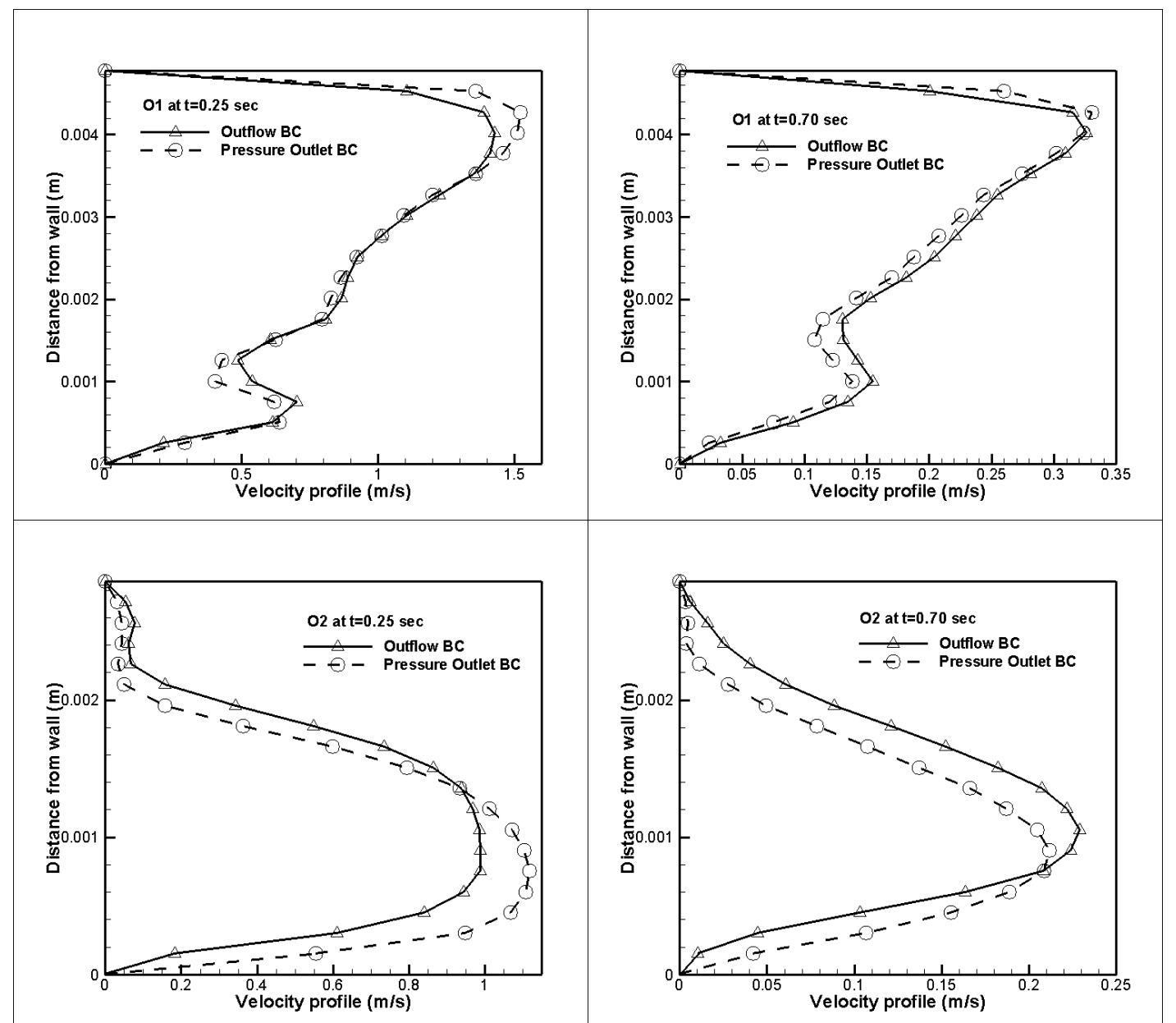

Figure 4.29: Velocity profiles at different locations for Aneurysm-B: O1 at systole (top left) and diastole (top right), $\mathrm{O} 2$ at systole (bottom left) and diastole (bottom right) using different boundary condition

The results suggest that the flow dynamics depend on the $\mathrm{BC}$. The difference in the scalar concentration profiles resolved by either $\mathrm{BC}$ at the outlets show a difference of less than $3 \%$ across the board (Figure 4.30).

A dynamic pressure difference of $28 \mathrm{~mm}$ of $\mathrm{Hg}$ in the parental artery was found to drive the flow through the aneurysm geometry at the peak-systole. The total pressure distribution across the aneurysm-B geometry is shown in Figure 4.31 (left) which is scaled to correspond to the typical resting arterial pressure. 


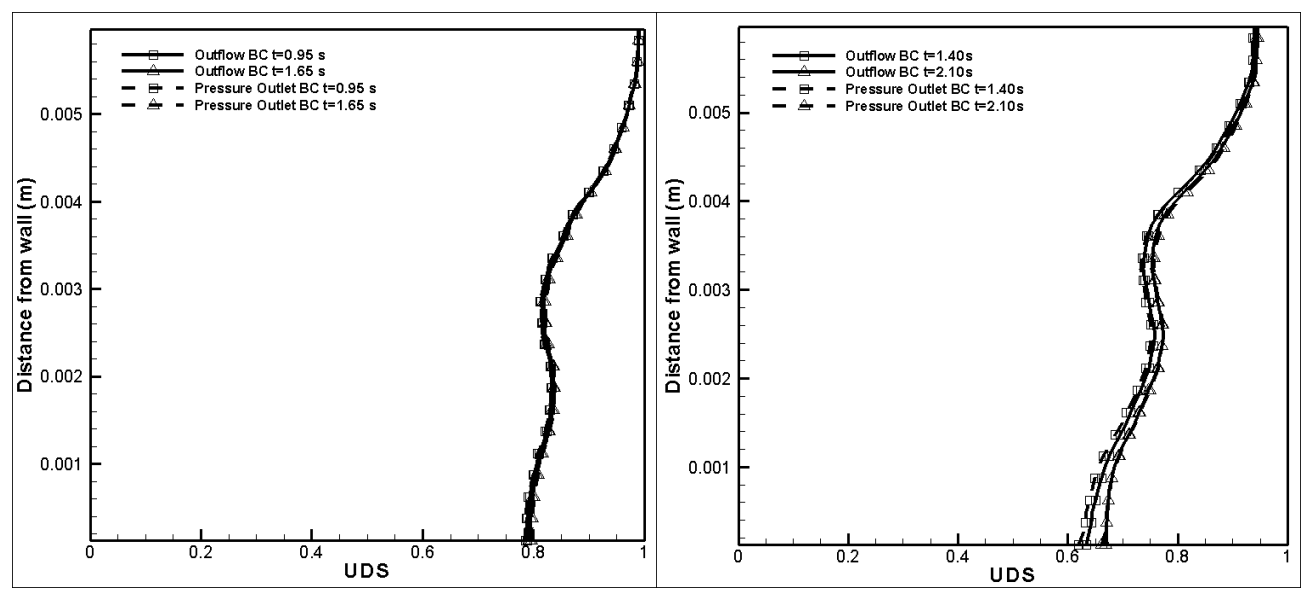

(a)

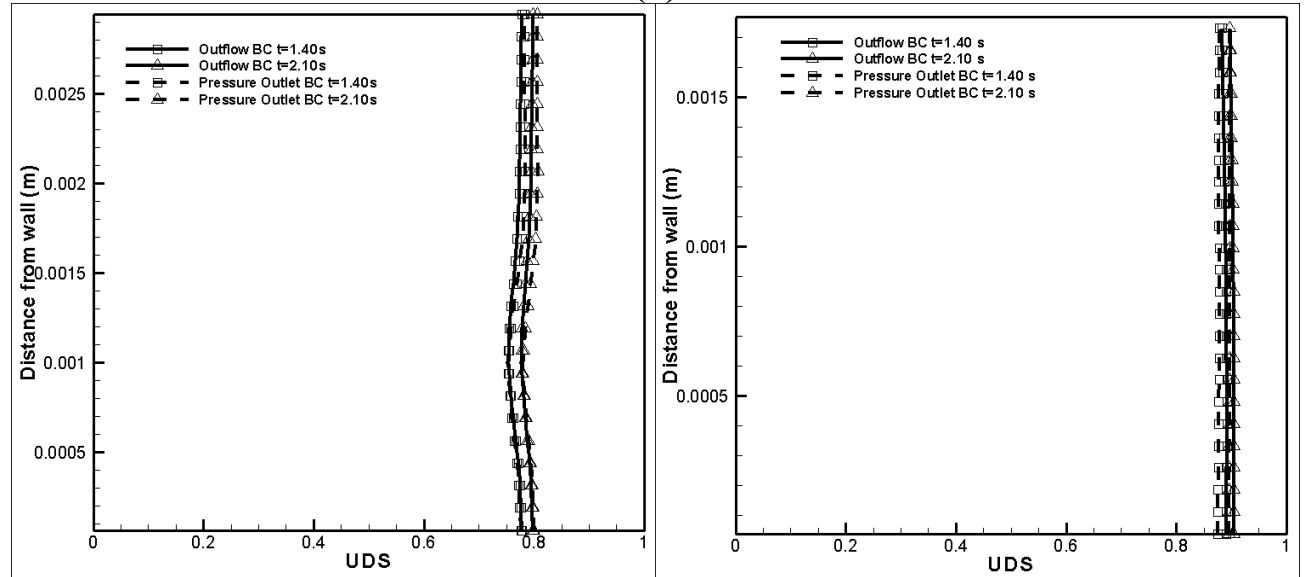

(b)

Figure 4.30: Scalar concentration at different locations for Aneurysm-B: (a) BF at systole (top left), BF at diastole (top right), (b) $\mathrm{O} 1$ at diastole (bottom left), and $\mathrm{O} 2$ at diastole (bottom right)

A drop in the arterial pressure occurs immediately after the aneurysm which is captured in detail in the middle and right of Figure 4.31, showing the pressure distribution in arterial cross-sections before and after the aneurysm. The larger size of the aneurysm-B and the existence of the throat constriction may be the possible causes for this relatively large pressure drop. In order to illustrate the pressure variation and the velocity profiles, they are plotted in Figure 4.32 as a function of time during one cardiac cycle at the geometric centers of the inlet cross section (PI) and the representative cross-sections of EA, $\mathrm{O} 1$ and $\mathrm{O} 2$. Under normal circumstances, one would expect to see a pressure wave at 
the outlets (two smaller arteries). The flat pressure profile is a consequence of prescribing a constant pressure at the outlets. This geometry has higher loses compared to the Aneurysm-A because it has more bends. This explains the accelerated flow in some sections of the geometry, compared to the velocity at the inlet. The flow in these zones leads to higher wall shear stresses. The superposition of the velocity and the static pressure waveforms would show that they are nearly in phase which agrees with the Womersley number calculation.

While the line plots (Figure 4.29) show small differences in the velocities at the outlets between either boundary conditions, the differences are more pronounced in contours of velocity across the cross-sections $\mathrm{O} 1$ and $\mathrm{O} 2$. It should be noted that for a given outlet, the mass flow rate at the outlet is the same for both the BC. Hence, the velocities would show only a difference in the distribution rather than the magnitude.

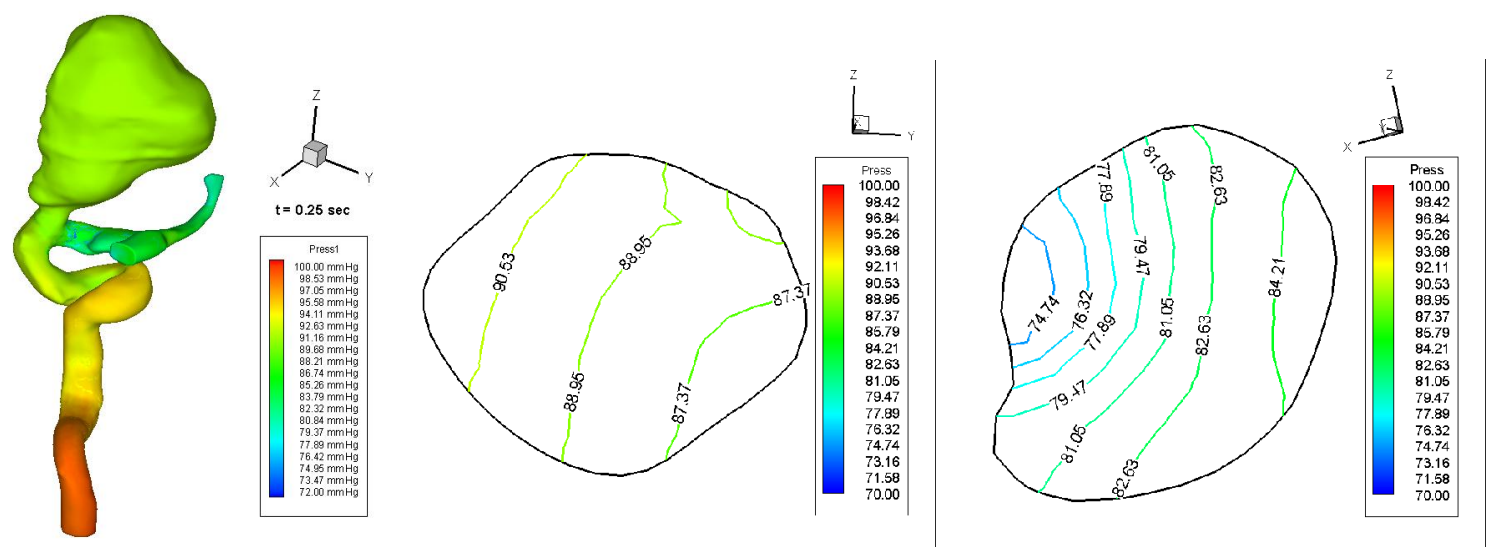

Figure 4.31: Total pressure (left) and static pressure comparison at cross sections planes immediately before (middle) and after (right) the aneurysm at the systole peak for Aneurysm B 

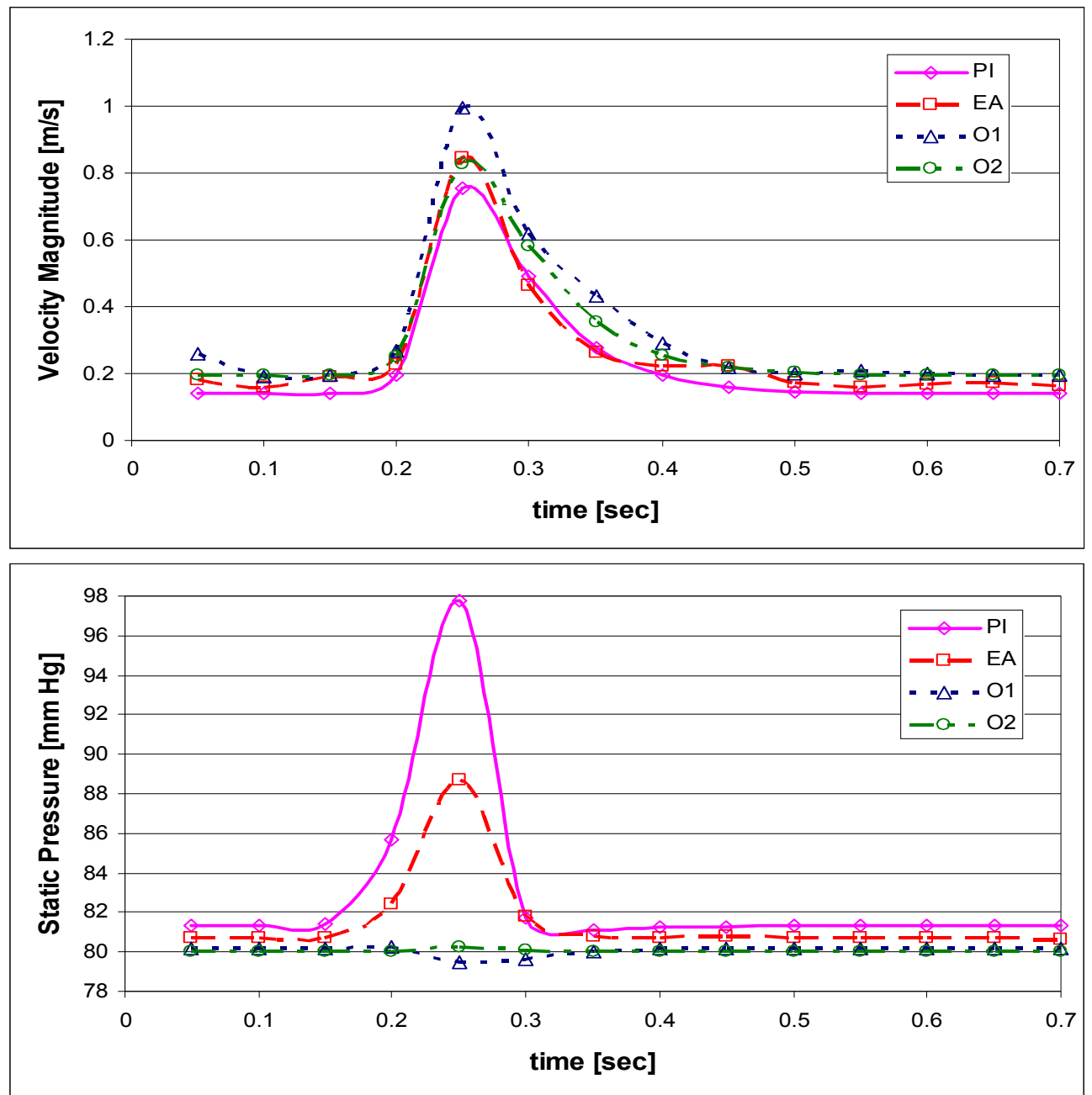

Figure 4.32: Velocity (top) and static pressure (bottom) waveforms at the geometric center of the inlet cross section and representative cross-sections PI, EA, $\mathrm{O1}$ and $\mathrm{O2}$ during one cardiac cycle for Aneurysm-B

This is evident from Figure 4.33, which shows the velocity contours at O1 (top left) and $\mathrm{O} 2$ (bottom left) obtained using the pressure outlets $\mathrm{BC}$ and $\mathrm{O} 1$ (top right) and $\mathrm{O} 2$ (bottom right) using outflow $\mathrm{BC}$. While the overall magnitude of the velocity remains the same, the velocity distribution shows significant differences, especially in the large outlet O1. This should lead to different distributions in the wall shear stress. 


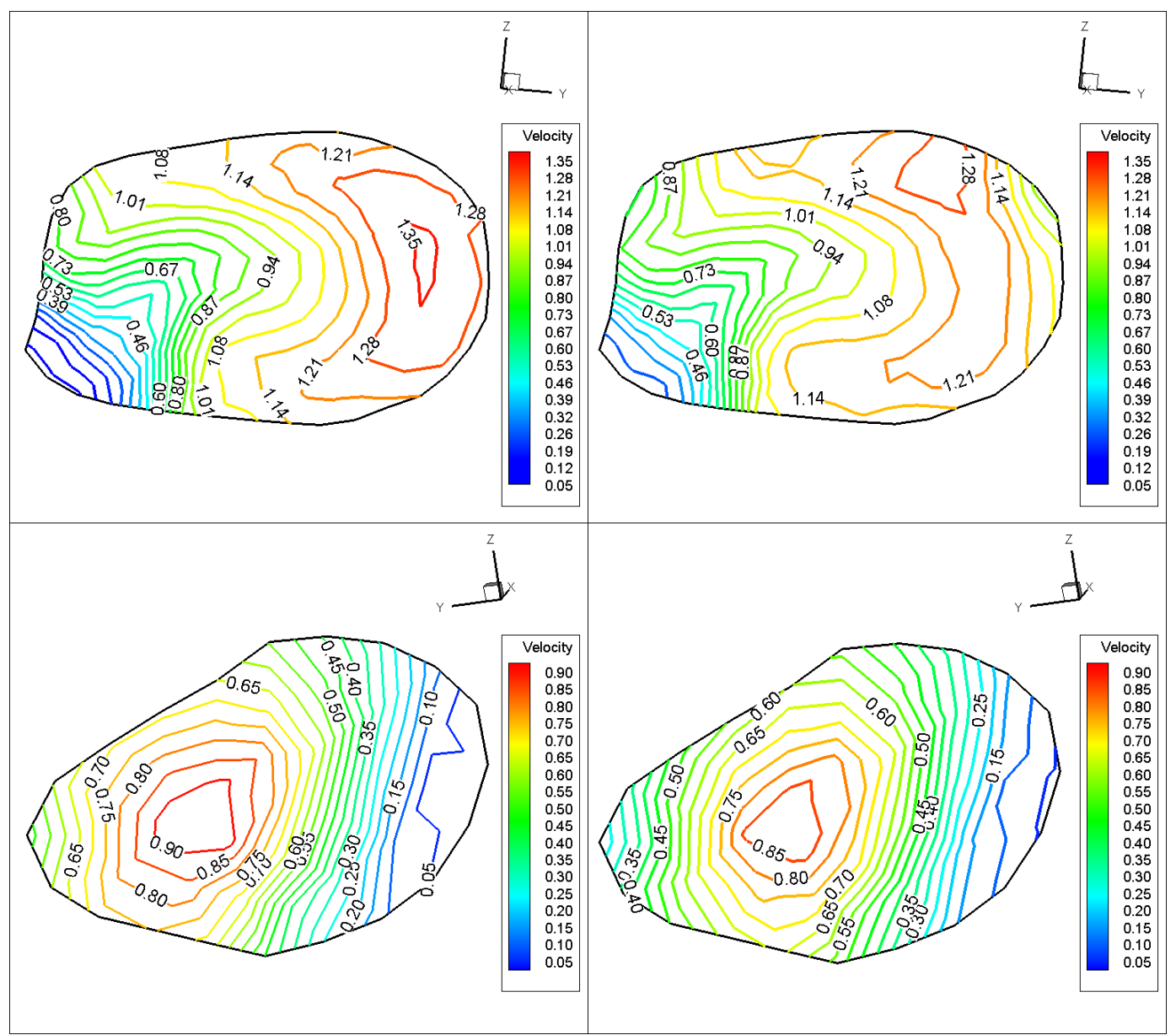

Figure 4.33: Velocity Contours at the systole peak $(t=25 \mathrm{sec})$ using different boundary conditions: pressure outlet boundary condition $\mathrm{O1}$ (top left), outflow boundary condition 01 (top right), pressure outlet boundary condition $\mathrm{O} 2$ (bottom left) and outflow boundary condition $\mathrm{O} 2$ (bottom right)

Figure 4.34 shows the pressure distribution at two outlets using the outflow BC. It must be noted that the pressure outlet condition assumes zero relative pressure at the outlets. With the primary goal of the CFD simulations being the characterization of pressure and velocity distributions, the choice of the boundary condition becomes critical for accurate results in these geometries. The outflow $\mathrm{BC}$ is believed to be the most adequate $\mathrm{BC}$ for $\mathrm{CFD}$ simulations related to arterial geometries. 


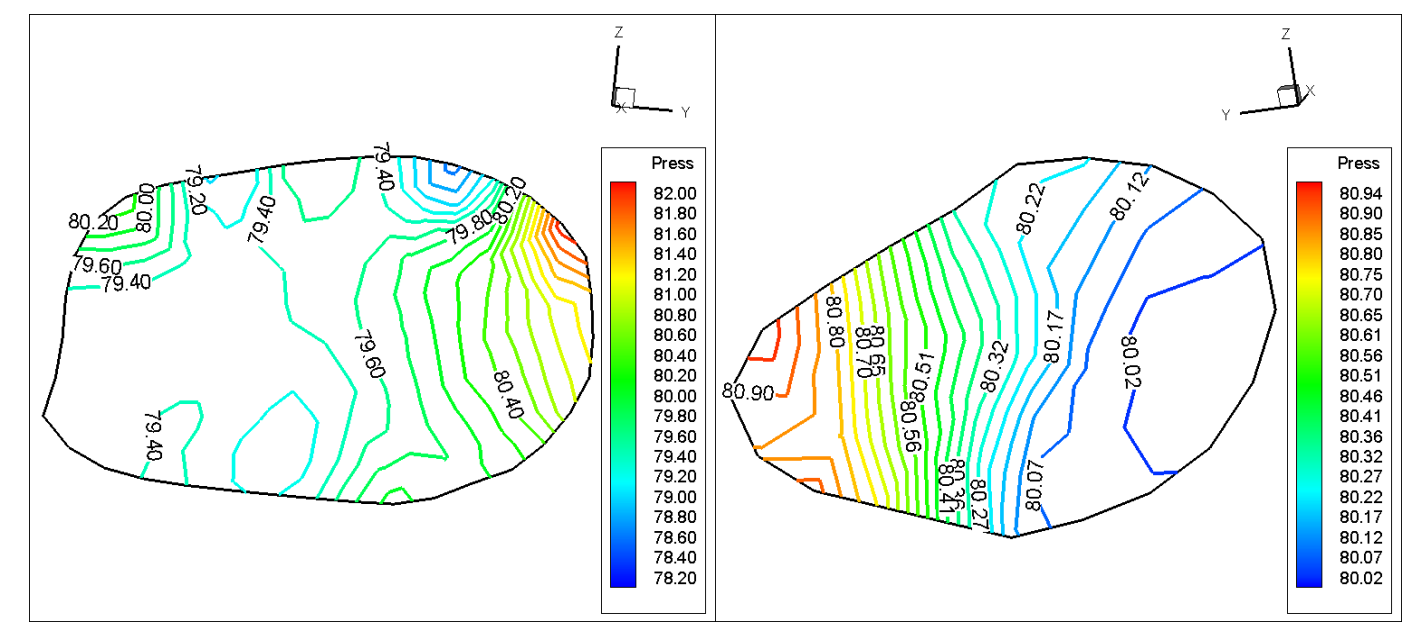

Figure 4.34: Static Pressure contours $(\mathrm{mm}$ of $\mathrm{Hg}$ ) at the outlets: $\mathrm{O} 1$ (left) and $\mathrm{O2}$ (right)

\subsubsection{Grid Dependence and Discretization Schemes effects for Aneurysm-B}

In a procedure similar to the one used for aneurysm- $\mathrm{A}$, pressure outlet $\mathrm{BC}$ conditions were imposed initially at both outlets in order to determine the volumetric flow rate for each outlet. This ratio was then used in a new simulation with an outflow BC. Results using pressure outlet BC for a second order QUICK discretization scheme gives a mass flow rate of $87.13 \%$ and $12.87 \%$ for the $\mathrm{O} 1$ and $\mathrm{O} 2$ cross sections respectively. While the mass flow rate results using pressure outlet $\mathrm{BC}$ using first order upwind discretization scheme were $86.65 \%$ for the $\mathrm{O} 1$ cross section and the rest (13.35\%) for the $\mathrm{O} 2$ outlet cross section imposed for outflow BC were $87.13 \%$ and $12.87 \%$ for both simulations. In the following procedure to quantify the effect of different discretization schemes on resolved velocity profiles, the outflow BC is imposed with the mass flow ratios of $81.13 \%$ and $12.87 \%$ at $\mathrm{O} 1$ and $\mathrm{O} 2$ outlet respectively. 
Figure 4.35 shows the difference in the velocity profiles at plane EA (top) during the systole peak (left) and the late diastole (right) using different discretization schemes. It can be seen that the maximum difference in the velocity profile between the first order and QUICK second order discretization schemes is about $18 \%$ at the systole peak and $20 \%$ at the late diastole. These plots also show that the velocity in the upper region of the profile is more prominent than in the lower region due to the presence of aneurismal flow entrance and free jets. The bottom plots of Figure 4.35 show the comparison of the velocity profiles at the BF location between the two different discretization schemes.

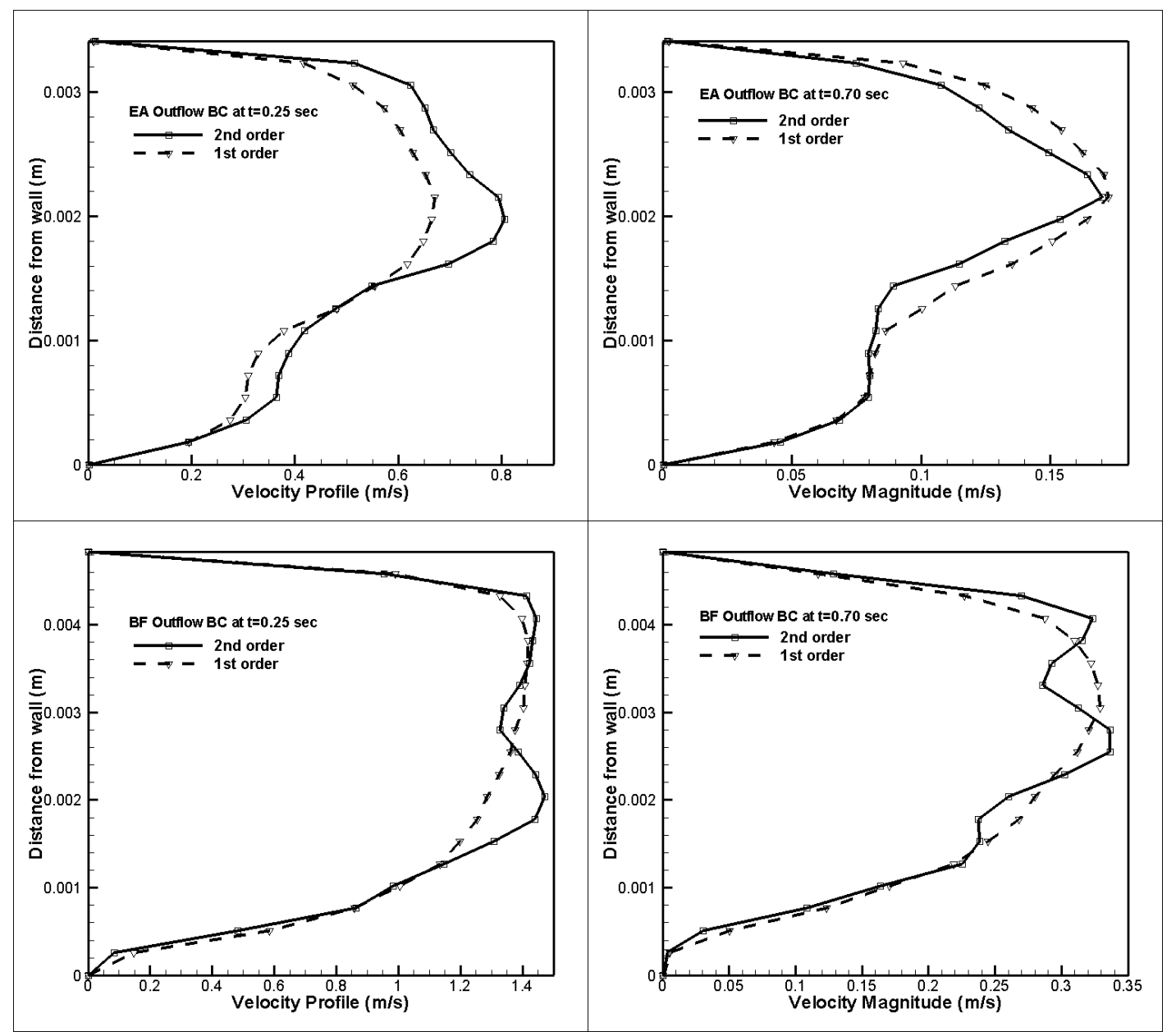

Figure 4.35: Velocity profiles comparison by using different discretization scheme at the EA cross-section (top) and BF cross-section (bottom) during the systole peak (left) and late diastole (right) for fine grid resolution 
At the peak of the systole (left) and the late diastole (right), the maximum difference is approximately $13 \%$ and $16 \%$, respectively. Overall, neglecting the second order term in the Taylor series expansions represents $15 \%$ difference in the velocity profiles approximately. On the other hand, the free jet getting inside the aneurismal dome would lead to inaccurate WSS calculation due to the not enough grid resolution close to the walls. The higher order terms of the discretization scheme also affects the accuracy of the flow characteristics specifically the secondary flows which occur at this plane.

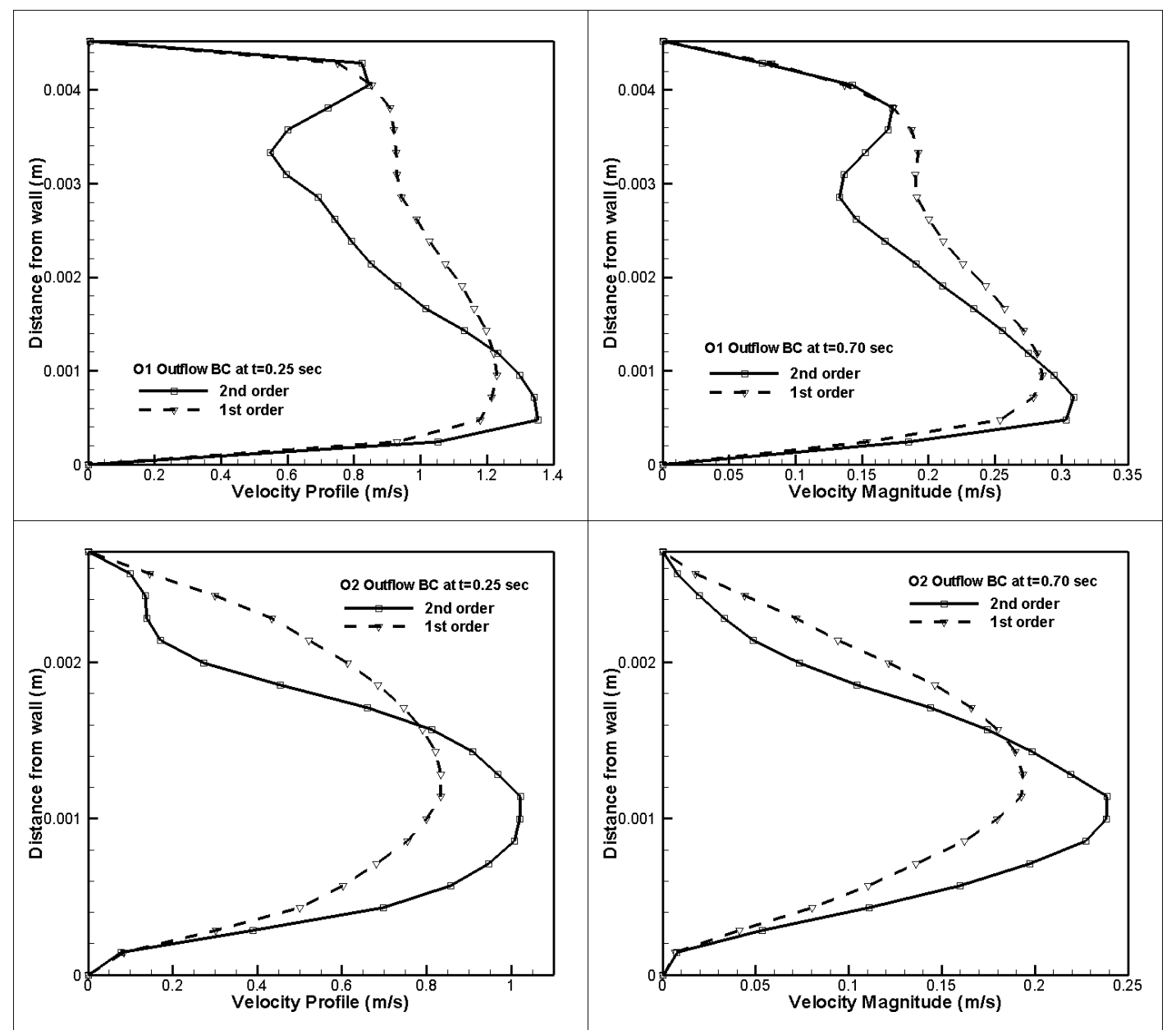

Figure 4.36: Velocity profiles comparison by using different discretization scheme at the 01 cross-section (top) and 02 cross-section (bottom) during the systole peak (left) and late diastole (right) for fine grid resolution 
The velocity profiles comparisons using different discretization schemes are also performed at the twin outlets $\mathrm{O} 1$ and $\mathrm{O} 2$ as shown in Figure 4.36. The plots on the top of the Figure 4.36 correspond to the $\mathrm{O} 1$ cross section where the difference is about $21 \%$ at the peak-systole (left) and 31\% at the end-diastole (right). The bottom part of Figure 4.36 shows the velocity profile comparisons at $\mathrm{O} 2$ cross section using first and second order discretization schemes. At $\mathrm{O} 2$ cross section, a difference of $60 \%$ at the systole peak (left) and $50 \%$ at the late diastole (right) is observed between the profiles. As seen in Figure 4.36, the higher order terms characterizes more details in the profile calculations, as reflected in the vortical flows computations. A similar trend was observed for aneurysmA geometry.

A grid convergence study is performed by using three different grid densities as shown in Table 3.1 in order to quantify the discretization error. Celik et al (2004) proposes a methodology to quantify the discretization error adopted by the ASME Journal of Fluids Engineering. This method is not only applied to the velocities profiles at various locations, but also to the WSS along a traced line as shown in Figure 4.9. This line traced along the region of interest is used to compare the WSS magnitudes among the three grid resolutions. The WSS profiles extracted by a line encompass the location of the maximum WSS and the aneurismal neck. The maximum WSS is included because this zone shows the highest sensitivity to the grid, as illustrated by Prakash's et al investigation (Prakash et al, 2001).

Velocity profiles comparison at planes EA and BF using three different grid resolutions as shown in Figure 4.37. The maximum approximate error in the profiles between the fine and coarse grid resolution at the EA cross section is $76 \%$ for the systole 
peak (top left of Figure 4.37) and 85\% at the end-diastole (top right of Figure 4.37).

These high error values are due to the presence of singular points where the error is very large due to an almost zero of the velocity profile difference $(\Delta \Phi)$ from grid to grid. Neglecting these singular points, the new approximate error is $36 \%$ and $22 \%$ for the peak-systole and end-diastole respectively. The extrapolated profile on a zero grid size in the EA cross section shows free jet at the peak of the systole. The extrapolated profile shows higher velocities in the upper region which leads to elevated impingement force at the dome of the aneurysm wall.
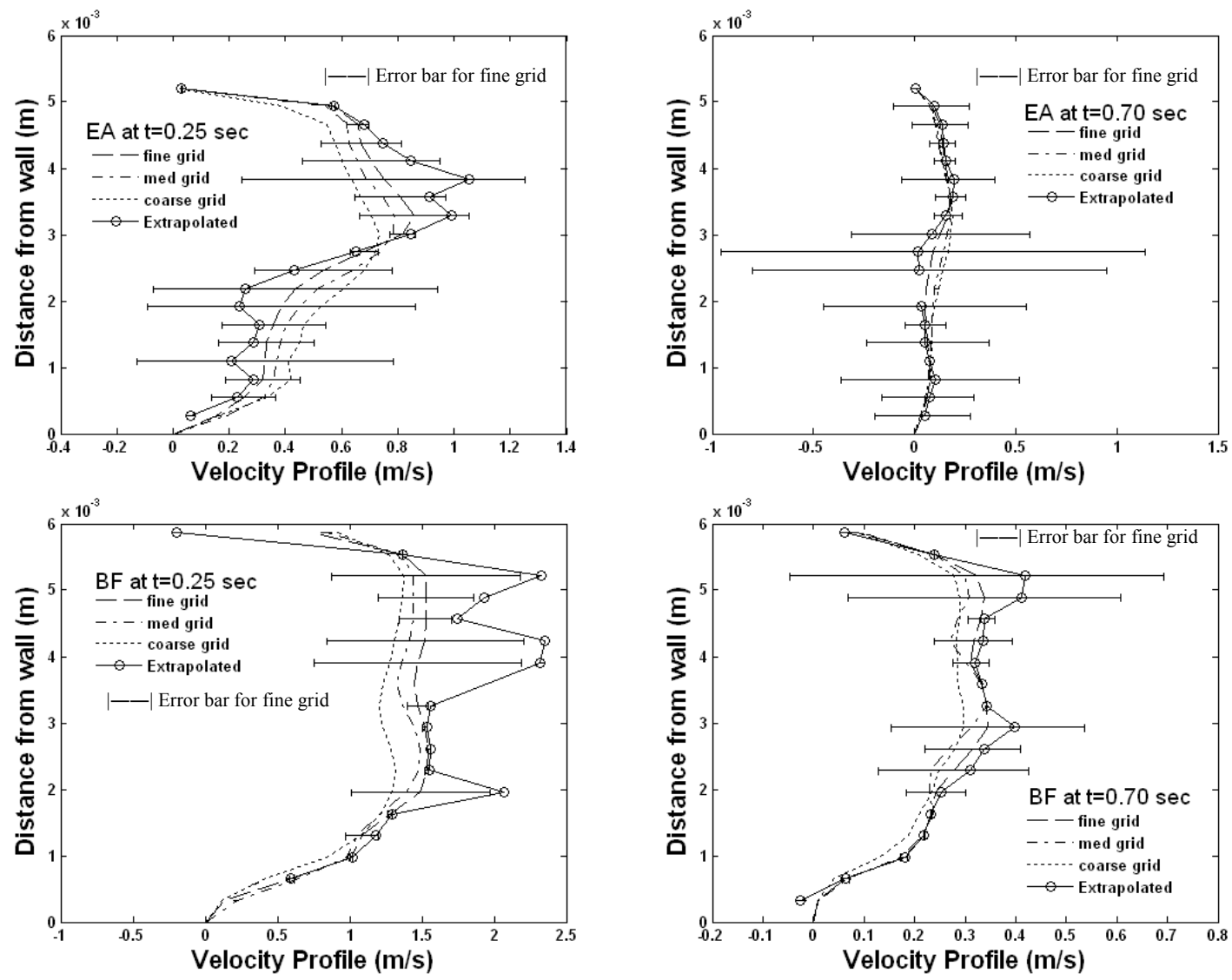

Figure 4.37: Velocity profiles comparison by using different grid resolution at the EA cross-section (top) and BF cross-section (bottom) during the systole peak (left) and late diastole (right) for second order discretization scheme 
Some singular points at the bottom of Figure 4.37 showing high error values are also neglected. Average discretization error values of $6 \%$ and $18 \%$ for the peak-systole (left) and end-diastole (right) are reported for BF cross section.

The velocity profiles at the $\mathrm{O} 1$ cross section as shown in at the top of Figure 4.38 demonstrate a maximum approximate error of $46 \%$ and $75 \%$ for the systole peak (top left of Figure 4.38) and the late diastole (top right of Figure 4.38) respectively. Neglecting the singular points, average approximate error values of $13 \%$ for the peak-systole and $20 \%$ for the end-diastole are reported. For the extrapolated velocity profile using zero grid size, the GCI method also shows higher velocities located at the core of the outlet plane. Similarly the approximate errors for $\mathrm{O} 2$ cross section as shown at the bottom of Figure 4.38. At the systole peak (bottom left), the maximum approximate error in the profiles is about $70 \%$, while at the late diastole (bottom right), the maximum estimated error bar is $64 \%$ approximately. If the singular points are not considered, the average error for the systole peak and the late diastole are $22 \%$ and $12 \%$ respectively.

A two fold increase in grid size leads to approximately a $10 \%$ difference in the velocity profiles. The error bars in Figure 4.37 and 4.38 are calculated using Richardson Extrapolation and give the grid convergence index $\left(\mathrm{GCI}^{21}\right.$ fine). Large error bars in the EA cross section at systole and diastole (top of Figure 4.37) suggests the inadequacy of the finest grid in resolving the large velocity gradients found in this region. In the vicinity of the EA cross section, various dynamic phenomena such as flow entering the aneurysm, flow exiting the aneurysm, local acceleration/deceleration due to the curve in the artery leading up to the region and an overall pulsatile flow, interact with each other to produce 
a highly complex flow field. The results and the error bars suggest that the simulations would benefit from a local grid refinement in this region.
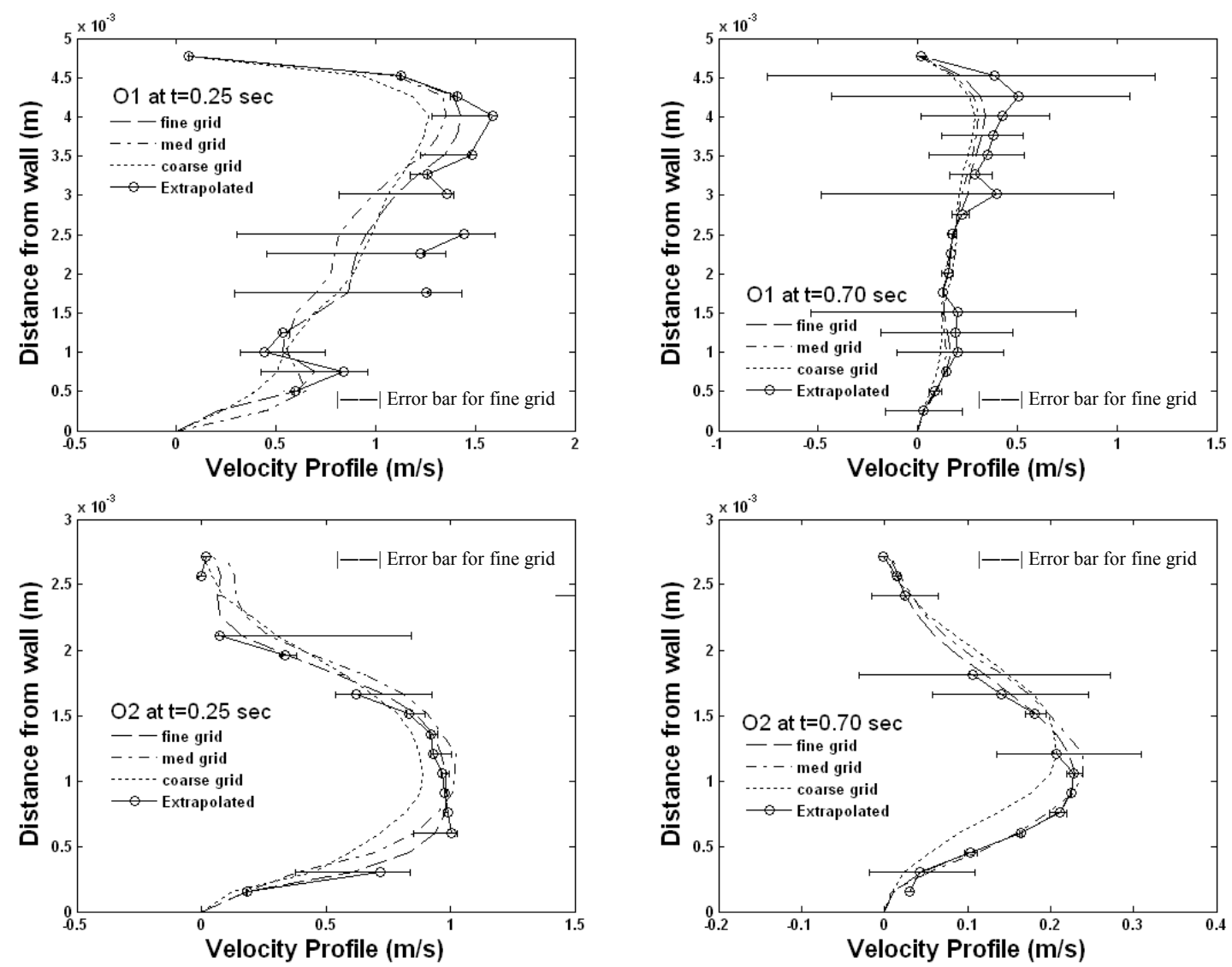

Figure 4.38: Velocity profiles comparison by using different grid resolution at the $\mathrm{O} 1$ cross-section (top) and $\mathrm{O} 2$ cross-section (bottom) during the systole peak (left) and late diastole (right) for second order discretization scheme

The WSS is plotted using three different grid resolutions in Figure 4.39. The location of the maximum magnitude of the WSS changes position accordingly to the grid refinement. The maximum WSS in the coarse mesh calculation occurs in the stenosis region (approximately 1-diameter downstream of the aneurysms location), while in the other grid resolutions (fine and medium), the location of the maximum WSS changes to a surroundings region of the aneurismal neck (downstream direction of the aneurysm). The 
maximum WSS location for the medium and fine meshes is placed in the same region as seen in Figure 4.39. This consistency in the location of maximum WSS between the medium and the fine grids also suggest the inadequacy of the coarse grid to resolve this flow. The maximum values exhibit changes of $45.10 \mathrm{~N} / \mathrm{m}^{2}, 34.05 \mathrm{~N} / \mathrm{m}^{2}$, and $24.56 \mathrm{~N} / \mathrm{m}^{2}$ for fine, medium, and coarse grid resolutions respectively at the systole peak. Figure 4.39 also shows a relatively high WSS on the concave region of curvature. Further analysis is presented in the next section comparing WSS when the aneurysm is removed.

Figure 4.40 shows the WSS profiles on a line traced along the artery surface close to the aneurysm throat at the systole peak (left) and the oscillatory WSS at three points on this line (right). It is to be noted that the point corresponding to the location of the maximum WSS (P3) is also plotted during the cardiac cycle. The left side of Figure 4.40 shows the extrapolated WSS profile for zero grid size and it exhibits an average error of $22 \%$. As Prakash et al (2001) claim, the maximum variation of the WSS profile is located at the maximum value. Moreover, the maximum error at this location is approximately $15 \%$ when compared between the extrapolated profile (GCI) and fine grid profile. Points P1 and P2 are located in the downstream and upstream of the aneurysm respectively. Figure 4.40 shows the variation of WSS with respect to the time at these three locations on the traced line (P1, P2, and P3). As expected, the results suggested cyclic variation of WSS at each of these locations in phase with the pulsatile flow. Even at these locations (P1 and P2) which do not show the overall maximum WSS in the geometry, WSS values oscillates between $15 \mathrm{~N} / \mathrm{m}^{2}$ to $2 \mathrm{~N} / \mathrm{m}^{2}$. However, P3 exhibit higher WSS changes during the cardiac cycle with the magnitude oscillating between in $42 \mathrm{~N} / \mathrm{m}^{2}$ to $5 \mathrm{~N} / \mathrm{m}^{2}$. 

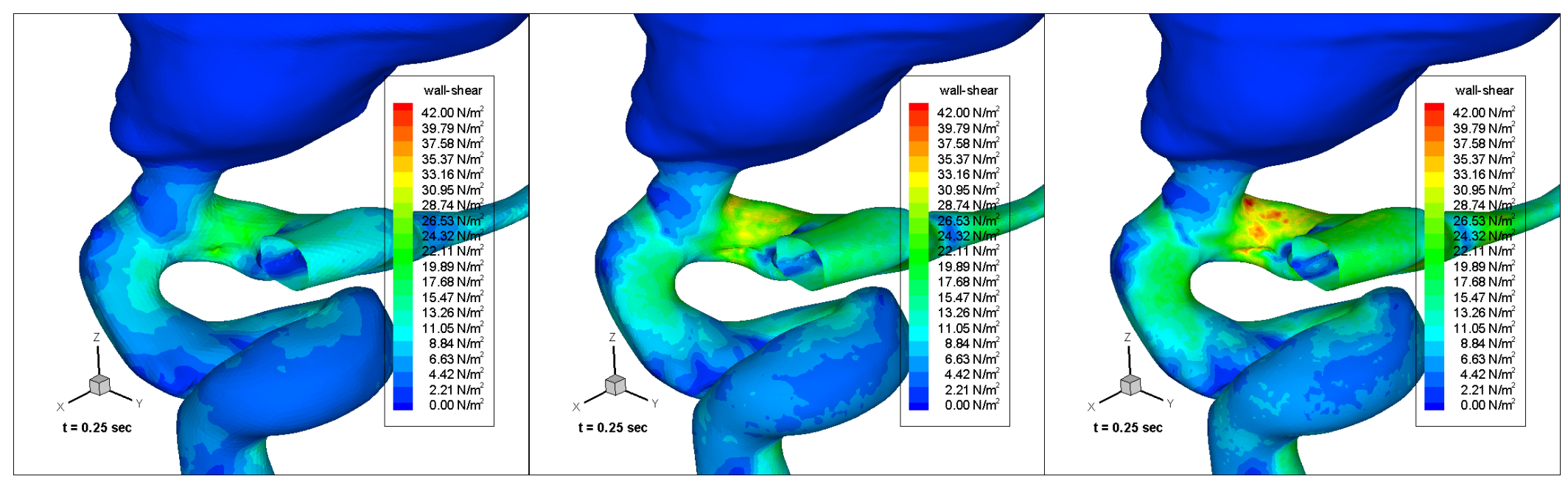

Figure 4.39: Wall shear stress contours for Aneurysm-B using different grid resolution at the systole peak: coarse, medium, and fine grid resolution
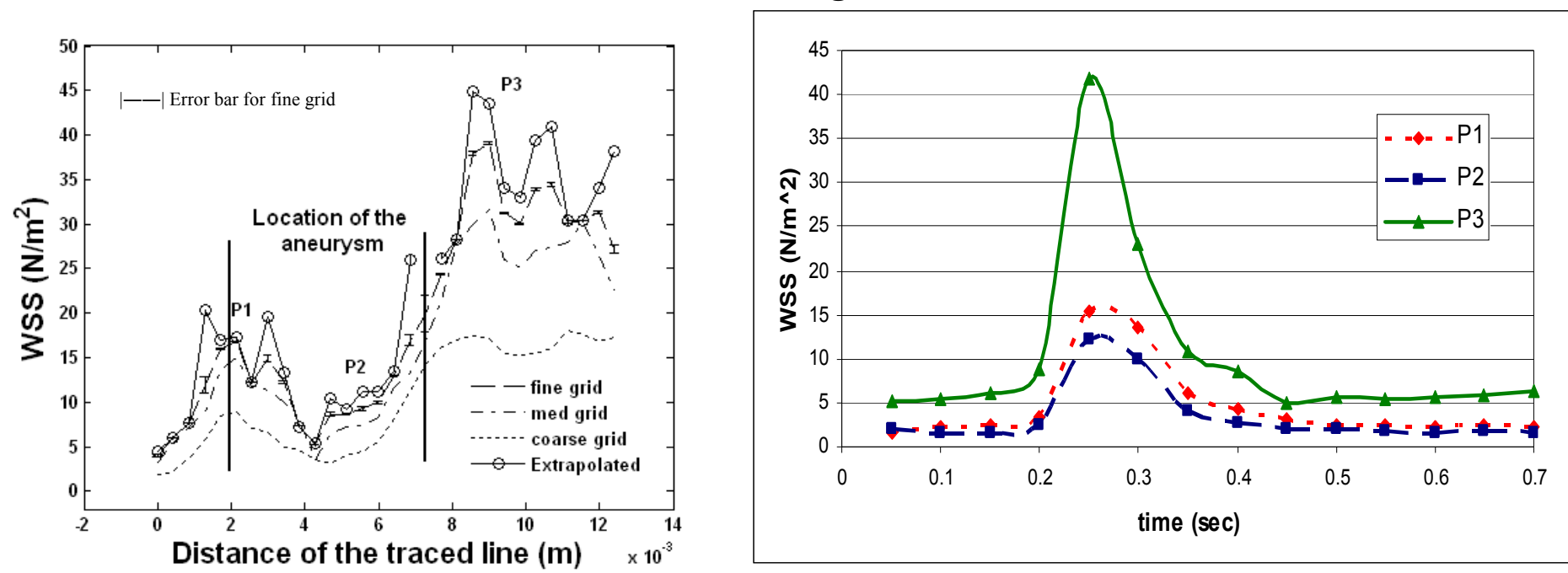

Figure 4.40: Results of the WSS along the traced line at the systole peak (left) and oscillatory WSS during a cardiac cycle at three defined points (right) for Aneurysm-B 
A detailed life cycle of the aneurysm is available courtesy of Dr. Ansaar Rai. The neurosurgeon performed a coiling procedure where the inside of the aneurysm dome was filled with platinum coil to suppress the progressive growth and to control flow impingement at the dome. Three months after the procedure, the aneurysm resurfaced due to the flow impingement in the throat region. CFD simulations can potentially fill this gap in information by providing the surgeon with a detailed flow simulation on a coiled aneurysm.

\subsubsection{Results for Geometry-B without aneurysm}

In a procedure similar to the one applied to aneurysm- $\mathrm{A}$, the disease is removed to find out the hemodynamic causes which may trigger the intracranial aneurysm. This geometry as geometry-A exhibits similar characteristics: categorized as side-wall aneurysms, aneurysm is located on the curvature of the artery preceding the bifurcation region (downstream direction) and it is close to the diseased. However, they differ in how the aneurysm is attached to the lumen vessel: a distinct neck region is present in aneurysm-B.

Similar to aneurismal geometry-A, three cross sections are selected to study the flow dynamics and compared on both geometries (with and without the aneurysm). Figure 4.41 shows the velocity profiles across a center line in the predefined cross sections using three grid resolutions. During the peak systole (left of Figure 4.41), the maximum approximate error for fine grid resolution is $9 \%$ at the BA cross section, $6 \%$ at the IS cross section, and 7\% at the AA cross section. While during the late diastole (right 
of Figure 4.41), these approximate errors in the fine grid velocity profiles are recorded as $24 \%$ for BA cross section, $11 \%$ for the IS cross section, and $15 \%$ for AA cross section. The higher errors during the end-diastole may be due to the volumetric flow ratio imposed (peak-systole rate) for all the cardiac cycle.
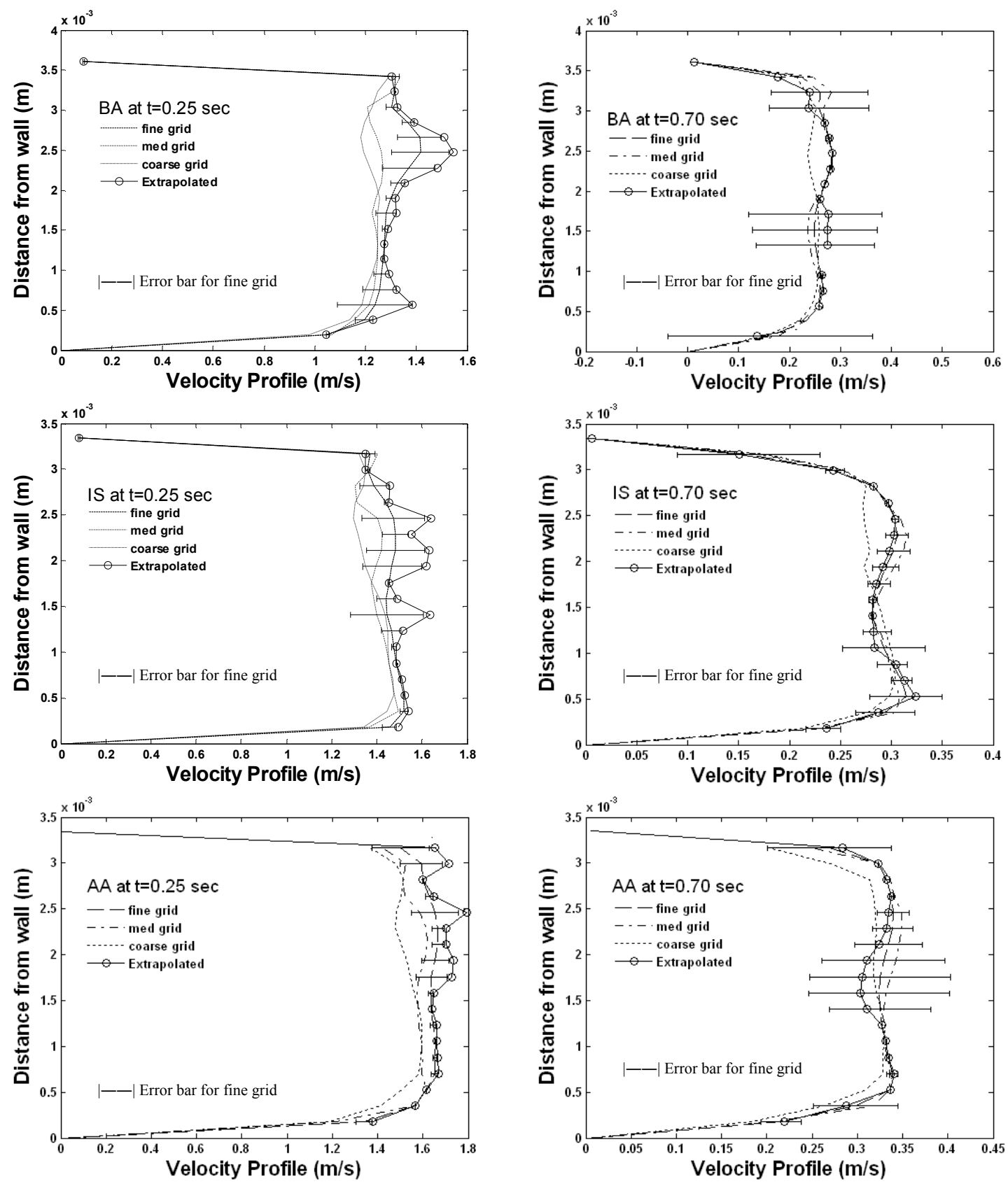

Figure 4.41: Velocity profiles comparison using different grid resolution during the systole peak (left) and late diastole (right) for BA, IS, and AA cross sections 
The removal of the aneurysm and the throat section leading to the aneurysm also removes the dynamic flow patterns entering and exiting the aneurysm leading to a more stable flow that is affected only by the acceleration/deceleration due to the curvature of the geometry, arterial bifurcation, and the pulsatile flow. In fact the pulsatile flow seems to have a larger effect on the velocity profiles than the arterial curvature and the bifurcation region. During the acceleration phase of the pressure pulse (systole), the flow is resolved with less error (left of Figure 4.41), while in the deceleration or stationary part of the pressure pulse (diastole) the calculation of the flow velocity includes higher discretization errors. Hence, a finer grid might be required to resolve the dynamics of the decelerating phase of the pulsatile flow. Special attention must also be paid to the pressure solver and the boundary conditions for the pressure term along with the mesh requirement to obtain a truly mesh independent solution.

Similar to the procedure followed for geometry-A, the WSS contours are also plotted using three different grid resolutions as seen in Figure 4.42. Unlike the geometry with the aneurysm, the location of the maximum WSS remains the same across the three grids in the constriction zone (1-diameter in the upstream direction of AA section). Pedley (1980) had made several studies of arterial bifurcation in pipes and describe how the parental artery splits in two child-arteries. He assumed that the cross section of the artery exhibits a circular shape all along the main vessel and it start getting flat at the poles. The creation of the two child-vessels is attributed to the flatness. The present geometry describes the similar bifurcation process, but the diameter is reduced on the south pole located before of the arterial division. It may be the case that arteriosclerosis leads to accumulation on this region, which decreases the arterial diameter (stenosis 
region). The maximum WSS values found for the three grid resolutions are $71.78 \mathrm{~N} / \mathrm{m}^{2}$, $50.21 \mathrm{~N} / \mathrm{m}^{2}$, and $46.53 \mathrm{~N} / \mathrm{m}^{2}$ for fine, medium, and coarse mesh resolution respectively. Further analysis of the other curvatures of the geometry found that the diameters in these zones are higher compared to the neighbor diameters (before and after the curvature section). These regions may be potential zones for cerebral aneurysms, but differ geometrically because they lack an arterial division located downstream of the region of interest. Therefore, this section where the aneurysm is virtually removed would affect the flow characteristics by means of a reflected flow wave.

A new line is traced on the region of interest encompassing the region where the aneurysm was originally present and the constriction region as shown in Figure 4.9. The time responses of the WSS at selected locations on the extracted line are also presented in the left of Figure 4.43. Points $\mathrm{P} 1$ and $\mathrm{P} 2$ are placed in the upstream and downstream direction of the artery where the aneurysm was initially located. The error bars on the fine grid WSS is about $10 \%$ and the extrapolated profile using GCI technique show very small differences compared to the fine grid WSS. The maximum WSS magnitude obtained for the fine grid and the extrapolated value differs on $8 \%$ of error. The time response of this location (P3) WSS shows a cyclic change of values ranged between $68 \mathrm{~N} / \mathrm{m}^{2}$ and $8 \mathrm{~N} / \mathrm{m}^{2}$ for the systole peak and the late diastole respectively. This range is much higher compared to the case with the aneurysm. Hence, it seems that the cyclic stress reversal on the artery wall contributes to the change of the mechanical properties leading to the development of an aneurysm. 

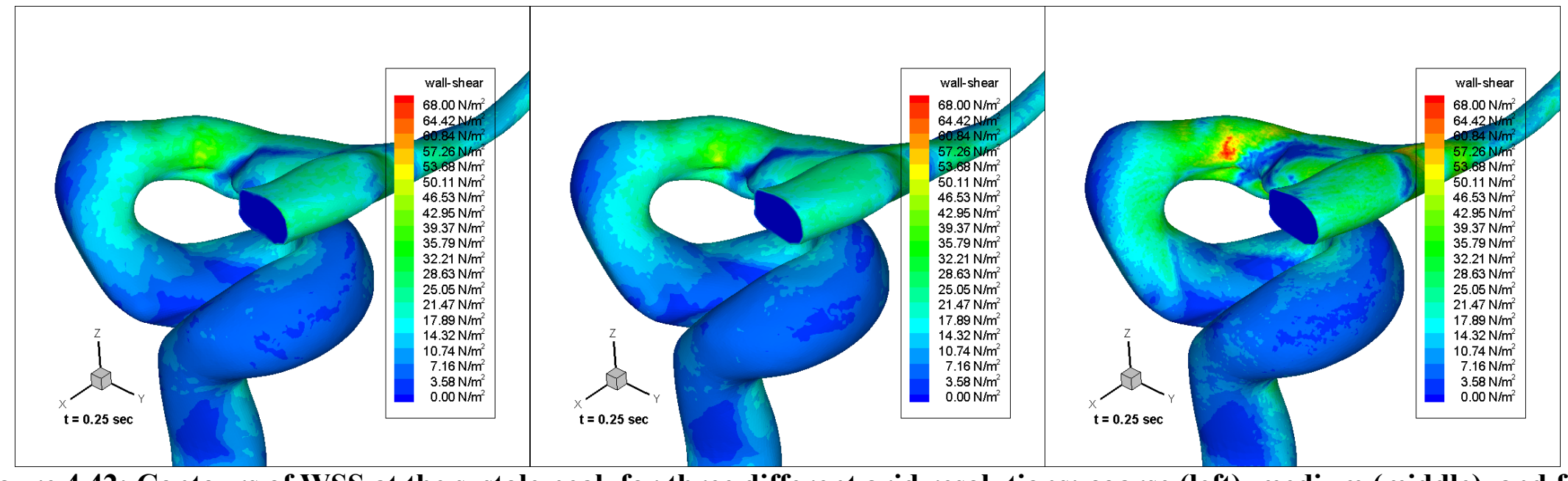

Figure 4.42: Contours of WSS at the systole peak for three different grid resolutions: coarse (left), medium (middle), and fine (right) grid resolutions
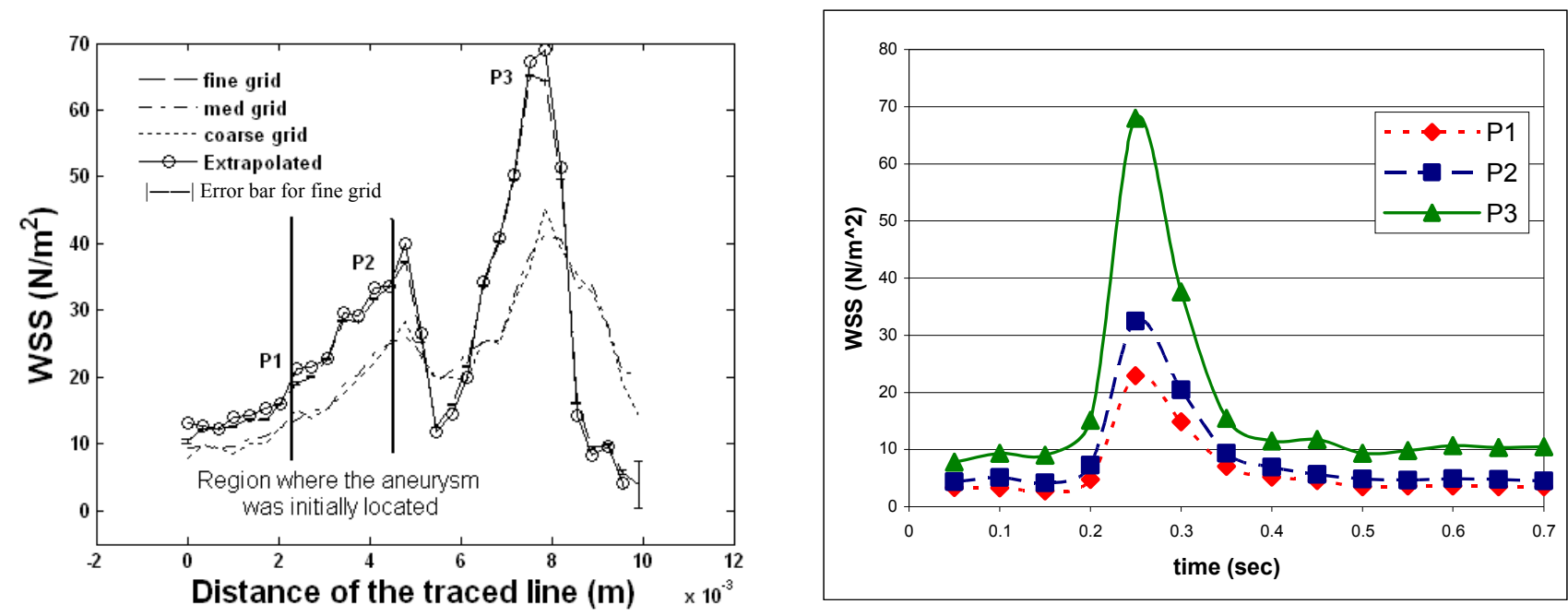

Figure 4.43: Results of the WSS along the traced line at the systole peak (left) and oscillatory WSS during a cardiac cycle at three defined points (right) for geometry-B 
The flow field is analyzed in the predefined cross sections to correlate the hemodynamic parameters that may contribute to the formation of aneurysms. The flow dynamics are observed using a fine grid resolution. Figures 4.44, 4.45, and 4.46 show the velocity vectors colored by the dynamic pressure contours at BA, IS, and AA cross sections on the region of interest. The comparison is performed for geometries with and without the aneurysm in order to establish the flow characteristics differences. Cardinal directions are used to explain the flow dynamics at these cross sections. Overall, the flow characteristics in the geometry with aneurysm are more complex than the ones for the geometry without the aneurysm due to the entering/exiting flow. The flow dynamics on the region of interest for the geometry without the disease show strong recirculation vortices induced by the arterial curvature.

The velocity vectors colored by the dynamic pressure at BA cross section is shown in Figure 4.44 corresponding to the geometry with (top) and without the aneurysm (bottom). At the peak of the systolic phase, the flow impinges on the north east region of the artery wall for the geometry with the aneurysm, forming a clockwise vortex located in the south region. Strong flow vectors going into the aneurysm are clearly observed in top left of Figure 4.44. At the same time (systole), the geometry without the aneurysm shows flow impingement on the north-east part of the artery wall where the aneurysm would grow. When the deceleration phase starts, the flow continues into the aneurysm in the north east region artery wall with less force and the recirculation vortex in the south starts dissipating for the diseased geometry. At the same instantaneous time recorded $(t=0.30$ sec), the flow dynamics at this cross section for artery without aneurysm changes significantly. 


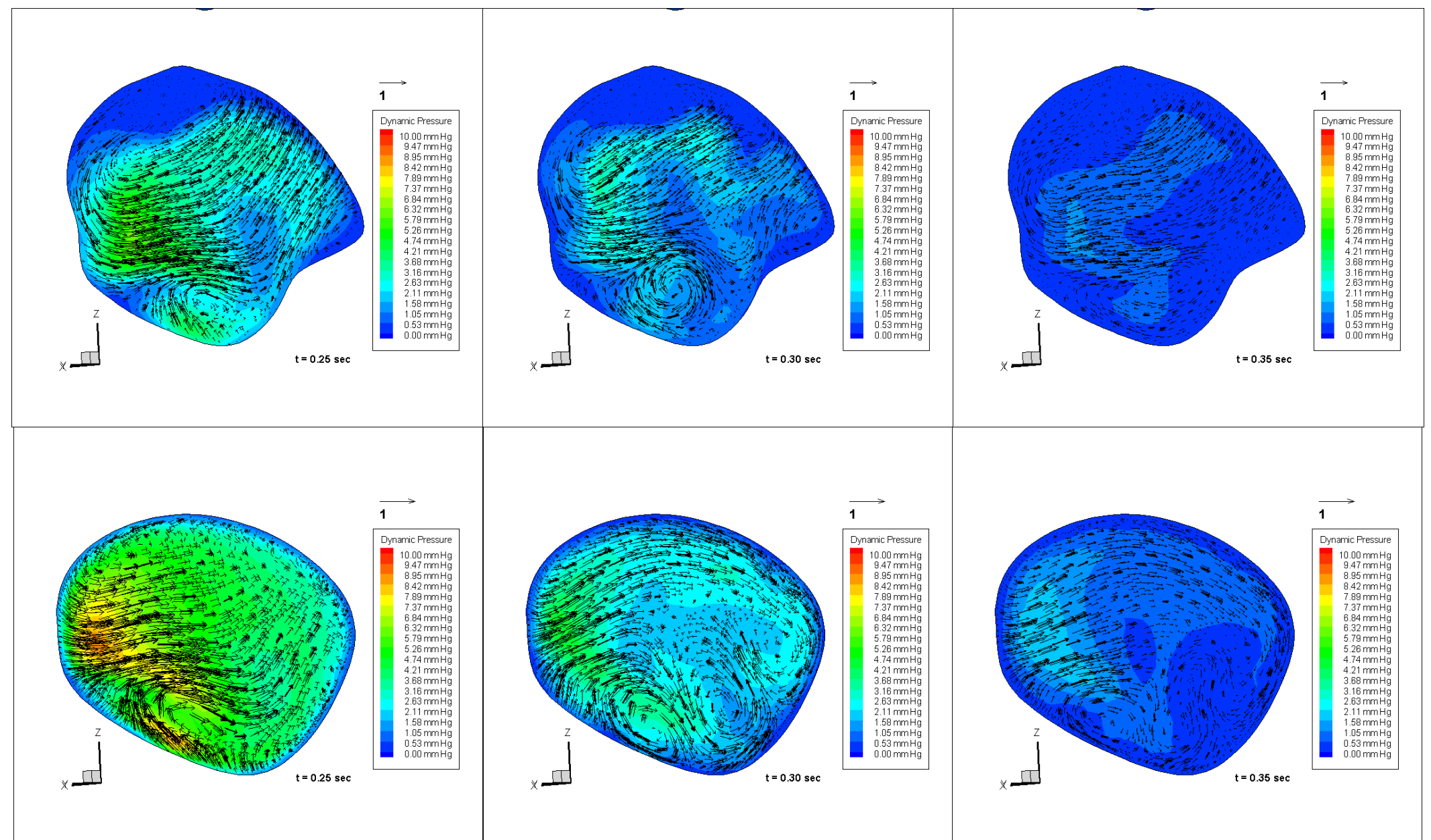

Figure 4.44: Velocity vectors colored by the dynamic pressure contours ( $\mathrm{mm} \mathrm{Hg}$ ) for BA cross section with the aneurysm (top) and without the aneurysm (bottom) 
Three vortexes are found at the plane: two strong vortical flows are located at the south region and the third is placed at the east zone (weak vortex). Moreover, the flow impacts in the entire north region starting from the west to the east. The dynamics of the flow for the previous time shows that the flow is driven form west to the core and later to the northeast region as shown in Figure 4.41. The flow characteristics have changed significantly on the north west zone where the flow direction reverses for the geometry without the aneurysm. At the late systole $(\mathrm{t}=0.35 \mathrm{sec})$, the velocity has decreased considerably and the vortexes have diminished in both geometries. Only the geometry without the aneurysm shows some secondary flows located at the south zone. The change of flow direction as seen at the systole peak and the latter time plus the flow impingement may degenerate the wall properties leading to the formation of cerebral aneurysms.

Figure 4.45 shows the flow characteristics colored by the dynamic pressure at IS cross section from the peak-systole through to the end-systole for the diseased geometry (top) and the reconstructed health geometry (bottom). The geometry with the aneurysm shows a strong clockwise recirculation at the south region at the systolic peak which dissipates at latter times. More vortices are found in the aneurysm region at several locations during the three times recorded. At the systole peak, a vortex can be observed at the south region which increases the velocity tangent to the surface. Thus, it would increase the shear stress on the wall in this region. At the opposite side of the cross section (north region), similar acceleration is seen close to the wall. This vortex impinges on the internal artery wall and the recirculation gets stronger at the south region of the cross section. 


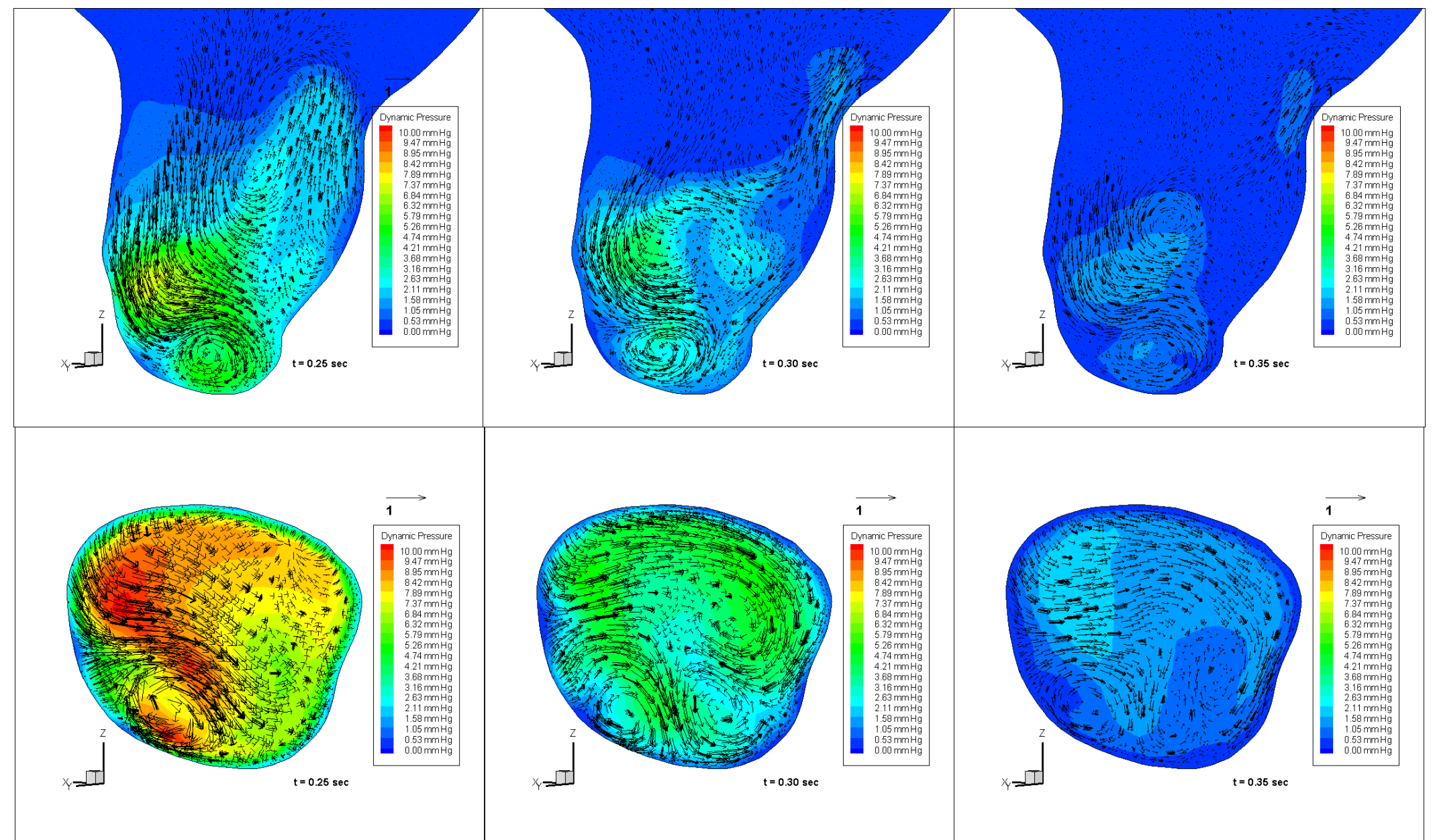

Figure 4.45: Velocity vectors colored by the dynamic pressure contours ( $\mathrm{mm} \mathrm{Hg}$ ) for IS cross section with the aneurysm (top) and without the aneurysm (bottom) 
In time, this flow impingement could start weakening the arterial wall causing lesions on the endothelial cells. Latter times recorded $(t=0.30 \mathrm{sec})$, the flow becomes more complex and two more recirculation regions are formed at the west zone of the cross section. These last two vortices started merging until they vanished when the systole reach its end. The vortex located at the south west region also start diminishing for the latter time $(t=0.35 \mathrm{sec})$, decreasing its vorticity.

The flow characteristics for the geometry with (top) and without the aneurysm (bottom) display variations at the AA cross section due to the aneurysm geometry as shown in Figure 4.46. During the systole peak, the flow is reentering the parental artery and forms a strong recirculation at the south region for the geometry with the disease. When the flow is decelerated $(t=0.30 \mathrm{sec})$, the formation of two strong vortices are observed at the core and the south west region of the cross section. In time, the vortex located at the core zone starts dissipating and it almost vanishes at the later instantaneous time recorded $(t=0.35 \mathrm{sec})$. The vortex located on the south region shifts its position to the south east region and the vorticity gets weaker in the deceleration phase. Figure 4.46 also shows the flow dynamics of the geometry without the aneurysm (bottom) starting from the systole peak. During the systole peak, the fluid flow characteristics differ from the previous cross section on the north east region. The flow enters the AA cross section from the west side and it is divided creating a clockwise vortical flow located in the south west region. The other flow fraction impinges on the south east region and moves upward the defined plane. A change of the flow direction is observed at the north east region when compared to the diseased cross section, where the flow moves in the downward direction (flow exiting from the aneurysm). 


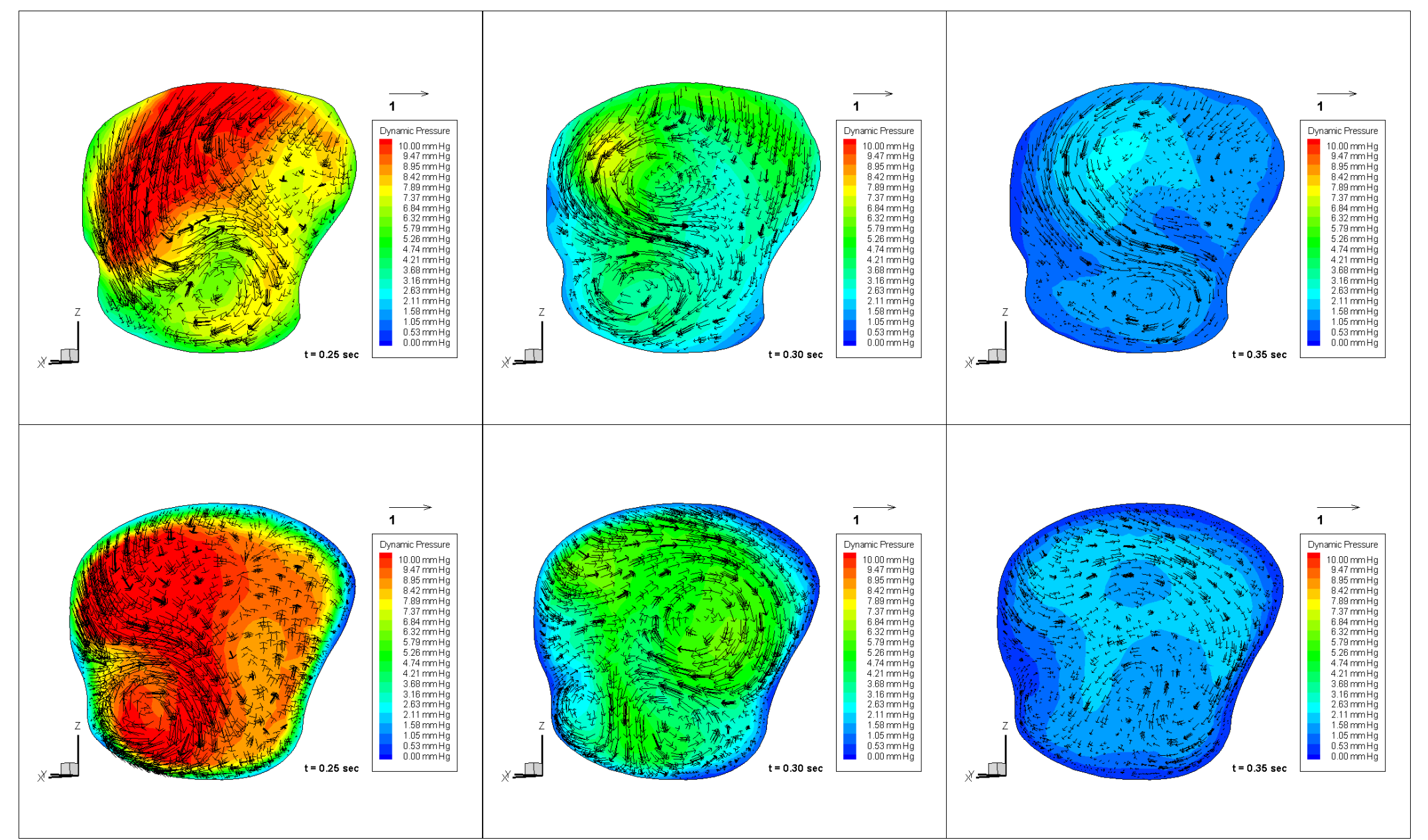

Figure 4.46: Velocity vectors colored by the dynamic pressure contours ( $\mathrm{mm} \mathrm{Hg}$ ) for AA cross section with the aneurysm (top) and without the aneurysm (bottom) 
Later instantaneous time record $(t=0.30 \mathrm{sec})$ shows the dynamics of the flow in which the formation of three vortexes is found at the same cross section. A strong clockwise recirculation flow is found at the core of the plane as a result of the flow impact form the north to the east interior wall, inducing the aneurysm formation.

The other two recirculation vortices are located at the south region, in which the fluid moves downward toward the convex region. These vortexes are mostly vanished in the later time $(t=0.35 \mathrm{sec})$ specifically the strong recirculation flow located at the core of the AA cross section. Similar to geometry-A, the aneurysm may develop due to the change of the flow direction at the concave region, provoking a continuous alteration of the endothelial cells structure. Experimental studies had conducted by Malek et al (1999) shows how the endothelial cells change according to the flow direction. In time, a continuous change of flow direction applied to the endothelial cells may provoke a weakening of the artery walls, hence leading to the origin of aneurysms. The most common location for aneurysm formation seems to be regions that exhibit arterial curvature.

The change of direction in recirculating flow also occurs at other locations such as that seen in the curved sections of the artery. Assuming the lumen vessel as a pipe with constant diameter as Himeno's investigation suggest (Himeno, 2007), the size of the curvature sections show an increment as compared to previous and subsequent cross sections. Therefore, the flow impingement (high WSS) and the change in recirculation flow direction in certain regions may provoke weakening of the vessels; thus forming potential regions for the aneurismal formation. The reflected wave flow due to the arterial bifurcation would increase the change of direction of the recirculation flow in the 
streamwise direction. This may be the reason why the aneurysms tend to occur close to bifurcation regions. The arterial bifurcation also affects the fluid flow characteristics as Pedley (1980) claims. This wave has been observed in experiments using elastic pipes without discontinuities along the channel.

High WSS seems to contribute to the origin of intracranial aneurysms as shown in the current investigation and corroborated by medical experience. However, low WSS seems to be a reason why the aneurismal dome keeps growing. The WSS analysis has been performed focusing on the WSS values at 5-diameters upstream and downstream direction of the region of interest. Maximum WSS location (stenosis region) can lead wrong conclusions. The flow dynamics inside the dome shows recirculating vortices changing the direction as shown in Figure 4.8. It seems that once the properties of the wall had changed (artery diseased), only these vortexes may provoke rupture at these regions where the flow change its direction. Further studies are needed to find out potential rupture zones and what hemodynamic factors may produce its rupture. Therefore, physicians would be aware of these regions when the disease is treated, assuring better handling cerebral aneurysms. 


\section{Summary}

The hemodynamic factors that influence the aneurismal formation and development are currently unknown. Many investigations had been carried out leading to ambiguous hypothesis. The most controversial parameter is the wall shear stress which is highly debated among the researchers. As a consequence, two main hypotheses presently exists on why cerebral aneurysms occur, viz. high WSS triggers aneurysms and low WSS triggers aneurysm growth. Therefore, the current investigation gives insight to the possible hemodynamic factors that can cause such disease. Through the use of pseudoaneurysm (pipe bend) geometry and real aneurysm geometries, the flow dynamics are studied on various cross sections to determine the potential causes of the wall deformation.

Several cases had carried out to investigate the flow dynamics that may cause the origin and development of aneurysms in pseudo-geometries and aneurismal geometries. Table 5.1 shows the summary of all the cases run for the geometries. A preliminary study is performed in pseudo-aneurysm geometries (bent pipe) to understand the flow dynamics. These geometries would elucidate the possible factors which trigger the aneurysms. Two different configurations yield to investigate the flow characteristics without the inclusion of the twist and several bend regions compared to real geometries. Additionally to the configuration, no bifurcation zone is included in the constant diameter geometry. Latter, a spherical bulge with smoothed edges is incorporated in the geometries to compare the flow dynamics. This initial approach will elucidate some important factors why the aneurysms may occur. Real geometries or aneurismal geometries are then studied using different grid resolutions and discretization schemes to quantify the 
differences and the discretization error. Finally, the removal of the aneurysm is performed in a process similar to the previous approach.

Table 5.1: Summary of all the cases for pseudo-aneurismal and aneurismal geometries

\begin{tabular}{|l|c|c|}
\hline GEOMETRY & \multicolumn{2}{|c|}{ PIPE BEND GEOMETRY (30 DEGREES BEND) } \\
\hline $\begin{array}{l}\text { with/without } \\
\text { aneurysm/bulge }\end{array}$ & WITH BULGE & WITHOUT BULGE \\
\hline GRID SIZE & FINE GRID & FINE GRID \\
\hline DISCRETIZATION & & \\
SCHEME ORDER & $2^{\text {nd }}$ order & $2^{\text {nd }}$ order \\
\hline Momentum eq. & QUICK & QUICK \\
Scalar & QUICK & QUICK \\
\hline CASE ID & PB30-1 & PB30-2 \\
\hline
\end{tabular}

\begin{tabular}{|l|c|cc|}
\hline GEOMETRY & \multicolumn{3}{|c|}{ PIPE BEND GEOMETRY (60 DEGREES BEND) } \\
\hline $\begin{array}{l}\text { with/without } \\
\text { aneurysm/bulge }\end{array}$ & WITH BULGE & \multicolumn{3}{|c|}{ WITHOUT BULGE } \\
\hline GRID SIZE & FINE GRID & COARSE GRID & FINE GRID \\
\hline $\begin{array}{l}\text { DISCRETIZATION } \\
\text { SCHEME ORDER }\end{array}$ & $2^{\text {nd }}$ order & $2^{\text {nd }}$ order & $2^{\text {nd }}$ order \\
\hline Momentum eq. & QUICK & QUICK & QUICK \\
Scalar & QUICK & QUICK & QUICK \\
\hline CASE ID & PB60-1 & PB60-2 & PB60-3 \\
\hline
\end{tabular}




\begin{tabular}{|c|c|c|c|c|c|c|}
\hline \multirow{2}{*}{\begin{tabular}{|l} 
GEOMETRY \\
with/without \\
aneurysm/bulge
\end{tabular}} & \multicolumn{6}{|c|}{ ANEURYSM-A } \\
\hline & \multicolumn{3}{|c|}{ WITH ANEURYSM } & \multicolumn{3}{|c|}{ WITHOUT ANEURYSM } \\
\hline GRID SIZE & $\begin{array}{l}\text { COARSE } \\
\text { GRID }\end{array}$ & MEDIUM GRID & FINE GRID & $\begin{array}{l}\text { COARSE } \\
\text { GRID }\end{array}$ & $\begin{array}{l}\text { MEDIUM } \\
\text { GRID }\end{array}$ & FINE GRID \\
\hline $\begin{array}{l}\text { DISCRETIZATION } \\
\text { SCHEME ORDER }\end{array}$ & $2^{\text {nd }}$ order & $2^{\text {nd }}$ order & $2^{\text {nd }}$ order & $2^{\text {nd }}$ order & $2^{\text {nd }}$ order & $2^{\text {nd }}$ order \\
\hline $\begin{array}{l}\text { Momentum eq. } \\
\text { - Scalar }\end{array}$ & $\begin{array}{l}\text { QUICK } \\
\text { QUICK }\end{array}$ & $\begin{array}{ll}\text { Upwind } & \text { QUICK } \\
\text { Upwind } & \text { QUICK }\end{array}$ & $\begin{array}{l}\text { QUICK } \\
\text { QUICK }\end{array}$ & $\begin{array}{l}\text { QUICK } \\
\text { QUICK }\end{array}$ & $\begin{array}{l}\text { QUICK } \\
\text { QUICK }\end{array}$ & $\begin{array}{l}\text { QUICK } \\
\text { QUICK }\end{array}$ \\
\hline CASE ID & AA-1 & AA-2 & AA-4 & AA-5 & AA-6 & AA-7 \\
\hline
\end{tabular}

\begin{tabular}{|c|c|c|c|c|c|c|}
\hline GEOMETRY & \multicolumn{6}{|c|}{ ANEURYSM-B } \\
\hline $\begin{array}{l}\text { with/without } \\
\text { aneurysm/bulge }\end{array}$ & \multicolumn{3}{|c|}{ WITH ANEURYSM } & \multicolumn{3}{|c|}{ WITHOUT ANEURYSM } \\
\hline GRID SIZE & $\begin{array}{l}\text { COARSE } \\
\text { GRID }\end{array}$ & MEDIUM GRID & FINE GRID & $\begin{array}{l}\text { COARSE } \\
\text { GRID }\end{array}$ & $\begin{array}{l}\text { MEDIUM } \\
\text { GRID }\end{array}$ & FINE GRID \\
\hline $\begin{array}{l}\text { DISCRETIZATION } \\
\text { SCHEME ORDER }\end{array}$ & $2^{\text {nd }}$ order & $1^{\text {st }}$ order $2^{\text {nd }}$ order & $2^{\text {nd }}$ order & $2^{\text {nd }}$ order & $2^{\text {nd }}$ order & $2^{\text {nd }}$ order \\
\hline $\begin{array}{l}\text { Momentum eq. } \\
\text { Scalar }\end{array}$ & $\begin{array}{l}\text { QUICK } \\
\text { QUICK }\end{array}$ & $\begin{array}{ll}\text { Upwind } & \text { QUICK } \\
\text { Upwind } & \text { QUICK }\end{array}$ & $\begin{array}{l}\text { QUICK } \\
\text { QUICK }\end{array}$ & $\begin{array}{l}\text { QUICK } \\
\text { QUICK }\end{array}$ & $\begin{array}{l}\text { QUICK } \\
\text { QUICK }\end{array}$ & $\begin{array}{l}\text { QUICK } \\
\text { QUICK }\end{array}$ \\
\hline CASE ID & AB-1 & AB-2 & AB-4 & AB-5 & AB-6 & AB-7 \\
\hline
\end{tabular}


In order to proceed with the simulations, the following assumptions are made. 1) Laminar flow is assumed to be during all cardiac cycle. 2) The walls of the arteries are assumed to be rigid. 3) Blood is assumed to have similar properties as liquid water (single phase) and 4) fully developed flow is assumed at the entrance of the parental artery.

Two aneurysm geometries are reconstructed from 2D images obtained by a rotational angiogram. Image processing techniques are applied to perform the geometrical reconstruction process are validated using another geometry (upper respiratory track) with similar reconstruction process. CFD simulations are performed to reproduce the fluid flow through the arteries using similar flow parameters. Due to the lack of information corresponding to the vitals of the patients, some additional assumptions are made so as to proceed with the investigation. The effect of different boundary conditions is investigated at the outlets using pressure outlet and outflow boundary conditions. Pressure outlet boundary conditions are used only to find out the volumetric rate at each outlet which are then applied in the outflow boundary conditions. This boundary condition seems to be the "right" compared to pressure outlet boundary conditions because zero gauge pressure is unlikely to occur in any part of the human body.

The effect of using first order upwind and second order (QUICK) discretization schemes are also studied to estimate the error in velocity profiles. Finally, grid dependence study is carried out using three different grid densities and the error is estimated through error bars for fine grid resolution. The GCI method is used to extrapolate velocity profiles to zero grid size which suggest a necessary grid refinement in the EA and BF cross sections. This complexity is due to the fluid dynamic response of 
the flow entering/exiting at the EA cross section, and the flow wave incident/reflected at the $\mathrm{BF}$ cross section for the geometry with aneurysm. As it is seen in the results section, this methodology is repeated for the arterial geometry with the dome (aneurysms) removed. This procedure is performed to revert in time and study the flow characteristics when the artery was healthy. Hemodynamic factors are investigated to determine the causes of origin and growth for side-wall intracranial aneurysms.

The WSS is another flow parameter studied in the current investigation and it is found that relatively high WSS may not be the only reason for the origin of aneurysm. The hypothesis of relatively high WSS as found in the results for both geometries is corroborated by several previous studies. In the current study, it is found that the changes of the vortical flows (vortex) in the radial direction may affect to the degeneration of healthy artery wall. These recirculation vortices are parallel to the primary flow (flow direction) due to the presence of the arterial bifurcation in the geometry. It is shown by many authors (Chandra, 1992; Pedley, 1977; Zeller et al, 1970) that a returning wave appears in an arterial bifurcation which rises in patients with hypertension. These wave reflections can be better observed in elastic pipes without the presence of any discontinuities such as the human arteries which exhibit wall compliance (Chandran, 1992). On the other hand, diseased arteries may be affected only by the secondary flows. Further investigation of this statement is required to prove this hypothesis. Healthy artery wall mechanics information is found in books and research papers; however, there is no data of diseased artery wall mechanics yet.

The endothelial cells (inner artery wall lining) change their structure due to the shear stress, which is the shear force applied by one layer on the next. Shear stress is 
caused by the friction force between molecules in sliding laminae (set of infinitesimal concentric shells). This shear force provokes the change of the structural intima layer to stimulate the secretion of continuous basal production of nitric oxide (NO). The loss of NO on smooth muscle proliferation, platelet activation, and leukocyte adhesion contributes to the evolution of atheroma (Levick, 2003). Atheroma or arterial plaque is accumulations of substances (fatty depositions) on the intima layer. Malek et al (1999) have performed experimental investigation on the arterial hemodynamic shear stress on bovines. During his experiments, high and low shear stress has applied to the endothelial cells during 24 hours achieving changes in their structure of the intima sheet as shown in Figure 5.1.

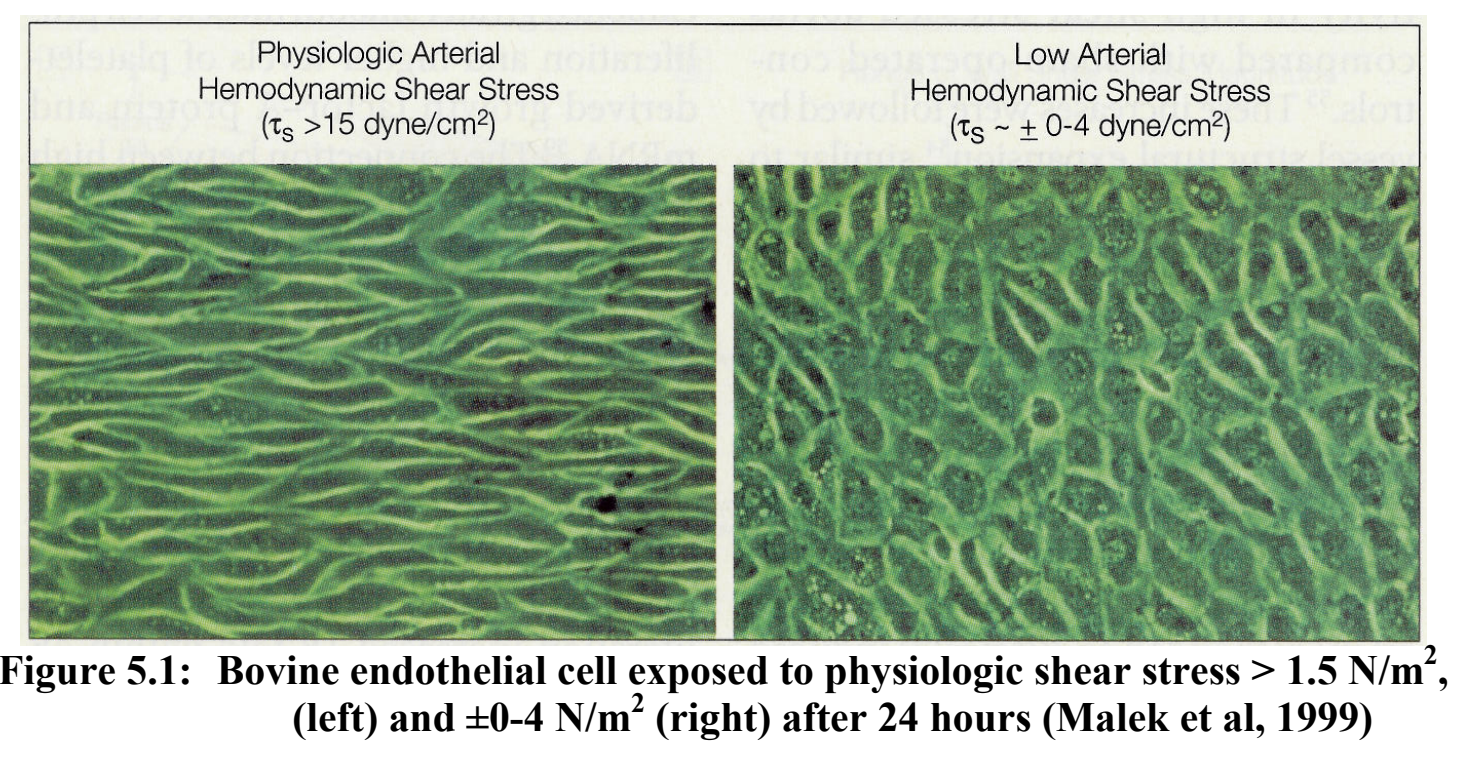

The weakening process of the endothelial cell starts when the blood flow collides with high velocity (centrifugal fluid force) on the concave region of the artery wall (curvature of the artery wall). Vortex flows are generated by the artery twist would increase the collision force at the same outer wall region at the systole peak. At the same 
time, the majority of the suspended cells may be accumulated on the convex region due to the slower velocities at this region of the artery wall. This effect is known as helical flow which is explained as a transfer of momentum from inside to outside of the bend. Similar nature occurs at the river bends, the vortex flow which is fast at the outer bank is steep and heavily eroded leading to the formation of meander. On the other hand, the vortex flow is slow at the inner bank shallow sand, gravel, and silt (Chant, 2002). When the deceleration phase starts, these vortices change the flow direction exerted at the concave wall region so the tugging force varies in magnitude reaching very low values. Hence, the intima layer would be constantly changing structurally due to the directional alteration of the vortices and this repeatedly process would provoke a weakening of the endothelial cells in healthy artery wall.

At the dome of the aneurysm, low WSS are observed on the wall. Even though low WSS are found in this region, there should be some relatively high WSS regions (considering only the aneurismal dome) where they may be potential zones of rupture. It may be the case that highly mobile (changing direction) and transient vortices at this region are enough to keep deteriorating the diseased arterial wall. Further studies are recommended to be performed inside the aneurysm to prove such statement. Solutions would show the flow dynamics inside aneurysms, and then it could be coupled with FEA (Fluid Solid Interaction-FSI) software to investigate the wall compliance. In order to perform this investigation, it is needed to be taken the mechanical properties of diseased artery wall is needed. Currently there is no such information available in the literature.

As mentioned before, it is not novel that the geometric configuration such as the arterial bifurcation and the curvature of the artery influence the flow. Complementary to 
the current investigation, laboratory experiments are conducted to calculate the mass flow rate in a bifurcation model. The experiment is repeated several times to avoid possible errors during the recorded measurements. Numerical simulation is carried out also to corroborate the experimental results using FLUENT software and to observe the returning flow wave. The results show strong agreement between these studies (see Appendix B). The possible differences between the results are due to uncertainty of the laboratory apparatus (flowmeter and beaker) and human errors.

\subsection{General Discussion}

Images obtained from angiograms are used to reconstruct realistic aneurysm geometries. The reconstruction is performed using an 'in house' software developed in MatLab, and the process is validated through rebuilding an upper respiratory track from cross sectional images (similar to the aneurismal images obtained from the angiography). The reconstruction results are also compared with those reported by Corcoran (2003). The current procedure shows more accurate dimensions compared to the reference values from Corcoran's study.

The flow patterns inside the aneurysm depend on the boundary condition chosen at the outlets if the outlets are close to the aneurysm. Careful consideration should also be given to the mass flow through multiple outlets and the corresponding boundary conditions. Outflow boundary conditions are suggested for flow calculation in arteries because the pressure variation with time at the outlet is not known in any part of the

human body. The use of outflow boundary conditions requires the volumetric flow rate at 
each outlet. This rate can be approximated analytically or as suggested shown in the result section of the current investigation. The variable volumetric flow rate obtained may have some influence in the final results using outflow boundary conditions, so it is recommended to use a dynamic feedback system to alleviate the flow rate at each outlet.

Many researchers including the current study have shown that the flow patterns depend critically on the geometric configuration of intracranial aneurysms. These most important geometric parameters are the diameter variation, constriction zones, arterial curvature, artery bifurcations, size, shape, and orientation of the aneurysms.

A grid convergence analysis is constructed and the CGI method recommended by ASME Journal of Fluid Engineering is applied to calculate error bars for fine grid resolution. This process has shown that the use of one grid resolution can lead to premature conclusion. For example, the maximum WSS for Aneurysm-B geometry is located on the upstream direction (close to the arterial bifurcation) of the aneurysm on the coarse grid. The refinement of the grid showed that the location of maximum WSS changes to a region close to the aneurysm (aneurismal neck) in the upstream direction.

The generation of suitable meshes in order to obtain accurate results on aneurismal geometries is a challenging problem. The flow parameter WSS is extremely sensitive to the grid resolution and it is exerted to the highest changes for every increment of the grid resolution. Calculate GCI values showed an averaged error of $30 \%$ and the location of maximum WSS varied significantly. Higher grid resolution would show significant changes in the WSS magnitude due to the slope variation in the velocity vectors near the artery wall (more cell elements). 
The occurrence of side-wall intracranial aneurysms seems to be a response to several hemodynamic factors, such as high wall shear stress, cyclical shear stress variation, and directional change of the secondary flows. The geometric configuration of the artery plays an important role in the flow dynamics as mentioned before. This statement is made by comparison of computational results at several cross sections in the region of interest with and without the cerebral disease. Upon removal of the aneurysm, it was observed (for both cases investigated) that the flow impinges on the concave arterial bend. This may lead to a weakening of the inner wall region and eventually lead to a formation of aneurysms. Moreover, the recirculating vortices are detected at the region where the aneurysm will occur in time, but they change direction during the deceleration phase. This change of direction is not only observed in a plane perpendicular to the flow direction, but also in stream direction due to the flow unsteadiness and the bifurcation region of the geometries (e.g. reflected flow). At the late diastole, the WSS magnitude diminishes to very low values (but remains oscillatory) so the shear force may change the wall structure (endothelial cells), leading to weakening of the intima layer. The degeneration of the intima layer would modify the wall mechanics in healthy artery walls which, in term, may eventually lead to aneurysms.

In general, curved arteries followed by an arterial bifurcation are potential regions for occurrence of side wall aneurysms due to the flow impingement and the recirculating vortices. These contribute to a high wall shear stress on the concave region of the arteries. This seems to be the reason why the artery usually exhibit larger diameter in the curved regions. 


\section{Conclusions and Recommendations}

\subsection{Conclusions}

Simple pipe geometries with arterial dimensions were tested using 30 and 60 degrees bends to asses the effects of curvature employing the same inlet boundary conditions. The results showed that higher curvature leads to higher WSS magnitudes and the maximum value is found at the inner convex region surface region of the bend with respect to the primary flow direction. Moreover, the curvature leads to strong flow impingement on the outer surface (where the bulge is expected to develop), provoking relatively high WSS. This flow impingement is observed more clearly at a plane towards the end of the bend with 30 degrees bending. When the bending angle is increased more complex flows are produced in the curved region.

In order to emulate an aneurysm in the pipe geometries, a protuberance is built onto the bend to elucidate the flow characteristics. The results showed that the maximum WSS occurs in the throat area (downstream direction) which validates previous observations in studies by Steinman et al, 2003; Feng et al, 2004; Jou et al, 2003; and Shojima et al, 2004. Other relatively high WSS magnitudes are also found in the curved region similarly to those found in the geometries without the bulge. This protuberance does not only increase the flow complexity in the curved region (changing the downstream flow pattern), but also the maximum WSS location in both geometries (close to the neck of the bulge in the downstream direction). Cross sections are analyzed inside the bulge in order to understand the flow characteristics. The results showed swirling flows inside the dome the strength of which depend on the phase of the cardiac cycle. 
Aneurismal geometries were reconstructed using an in-house code from 2D images obtained from CT clinical study. The advantage of using in-house code is the low development cost and higher control on the reconstruction procedure compared to any commercial software. Many researchers had created their customized codes in order to reconstruct their geometries and they had validated it using different methodologies. For example, Corcoran et al (2003) rebuilt an upper respiratory track and validated his results by comparison with values found in the literature. Using the same cross sectional pictures (Corcoran's courtesy), we had showed that the present methodology showed that is more accurate than Corcoran's methodology (error less than 5\%).

Simulations were also performed using two side-wall intracranial aneurysm geometries. During the systole peak, it was found for aneurysm-B that the location of the maximum WSS is located close to the throat (downstream direction). On the other hand, aneurysm-A geometry showed that the maximum WSS occurs at the stenosis region (about seven diameters upstream of the aneurysm location). This does not necessarily mean that high WSS is the cause of aneurysms: one needs to study the localized maxima. Also, it is important to study the hemodynamic parameters in the aneurismal surroundings. Relatively high WSS also occurs in the aneurismal surroundings specifically at the downstream direction of the aneurysm similar to the aneurysm-B geometry. The WSS also showed very low values at the diastole end phase, so the region of interest is exposed to large variations of the cyclical WSS, depending on the cardiac cycle phase. Secondary flows are also observed on the cross sectional planes of the curved region. The throat of aneurysm-B geometry increases the flow complexity. This 
complexity is due to the flow entering as a free jet and flow exiting from the aneurismal dome.

Finally, the aneurysm is virtually removed and simulations are carried out in order to go back in time and assess the causes of aneurismal formation. These results are compared with the ones obtained in the previous simulations (geometries with aneurysm). It was found that the primary flow impinges on the outer surface of the bend (where aneurysm was initially located) and high vorticity is observed at these cross sections. According to the cardiac cycle phase, the flow changes direction close to the wall where the aneurysm was originally formed. This process would lead to cyclic WSS produced by the pulsatile flow on the curved region. Secondary flows are also observed in the cross sections and they may play a significant role in weakening of the endothelial cells. Therefore, it can be concluded that the directional change of the flow, secondary flows, and the local maxima of the WSS may all play a role triggering intracranial aneurysms.

\subsection{Recommendations}

- In order to improve future simulations using outflow boundary conditions, a minor extension of the angiography routine has to be made whereby the mass flow rate at the inlet and outlets should be measured. This information would provide accurate data about the inflow and outflow to be imposed in the specific patient case for the hemodynamic flow predictions.

- Certain assumptions were made to perform the current investigation. These assumptions can be improved to represent the reality closely, such as: 
- The arterial walls are exerted by internal pressure, external pressure, and wall tension (nonlinearly visco-elastic properties).

- A velocity profile at the entrance is more likely to occur in arterial flows.

- Blood is a multiphase flow due to the suspended cells.

- Transitional flows may occur at the systole peak of the cardiac cycle. LES (Large Eddy Simulations) technique can be used to alleviate flows of transitional nature.

- The arterial volumetric waveform is more complex than the one used in the current investigation. Figure 6.1 shows a closer representation of a volumetric waveform during a cardiac cycle (Plesniak et al, 2007).

- The volumetric flow rate is calculated using pressure outlet BC. This rate changes according to the cardiac cycle phase, so a dynamic feedback system could be implemented to carry out simulations using outflow BC.

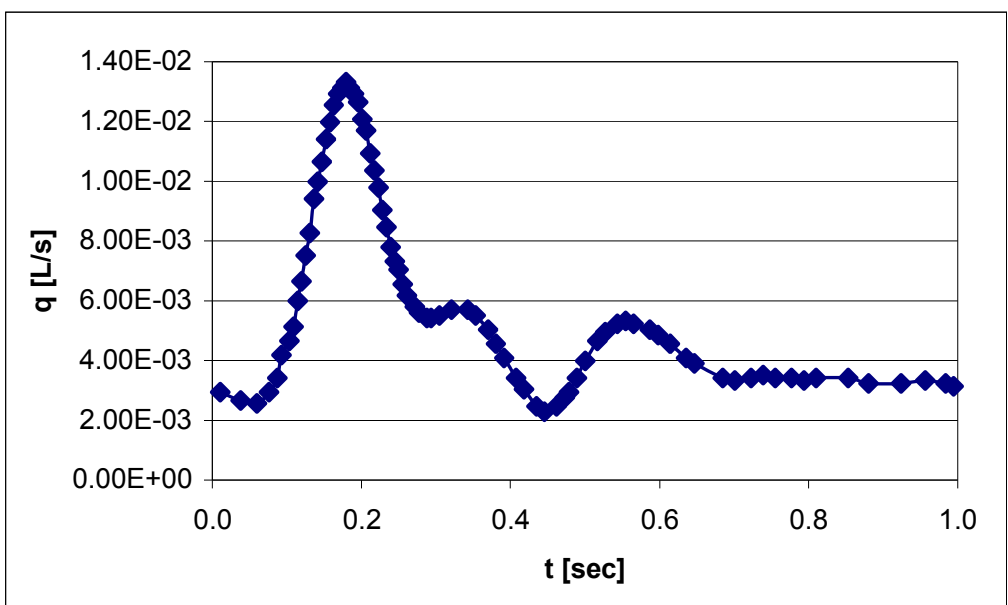

Figure 6.1: Specific-patient volumetric waveform for a cardiac cycle (Plesniak et al, 2007) 
- The application of the $\mathrm{BC}$ in aneurismal geometries may have some effect on the final results due to the proximity between the outlets and the aneurysm region. Further studies are recommended to find out the minimum distance of the outlet plane, so the outlet $\mathrm{BC}$ does not affect the final results. This effect can lead to premature conclusions in cerebral aneurysm's investigations.

- Adaptive grid resolution at certain locations (region of interest and arterial bifurcation) applied to the geometries with and without the aneurysm may lead to more stable and accurate results; however, the cost of computational time would increase considerably. The use of hexagonal grid shape may improve the computational time (reduction of the grid elements).

- Laboratory experiments of real cerebral aneurysm are very complex to be performed in human beings. Alternative techniques to validate the current results include carrying out experiments such as: 1) In vitro studies can be conducted by using transcranial Doppler techniques in a transparent silicon model connected to a special pump and 2) In vivo experiments can be also performed in rabbits (circulatory system similar than humans).

- The current work has elucidated the influence of some hemodynamic factors that may trigger cerebral aneurysms. Complementary FSI studies are necessary to corroborate such hypotheses focusing on the directional change of vortex flows and oscillatory high WSS in the region of interest. Mechanics of healthy arteries are well known and the coupling with this methodology would show some new interactive factors which may contribute to aneurismal occurrence. 


\section{Bibliography}

Acevedo-Bolton, G., Jou, L. D., Dispensa, B. P., Lawton, M. T., Higashida, R. D., Martin, A. J., Young, W. L., and Saloner, D. (2006). Estimating the Hemodynamic Impact of Interventional Treatments of Aneurysms: Numerical Simulation with Experimental Validation: Technical Case Report. Neurosurgery, 59(2), pp. 429-430.

Agarwal, G., Mozes, G., Kinnick, R. R., Gloviczki, P., Bruhnke, R. E., Carmo, M., Hoskin, T. L., Bennett, R. E., and Greenleaf, J. (2006). Vibrometry A Novel Noninvasive Application of Ultrasonographic Physics to Estimate Wall Shear Stress in Native Aneurysm. Ann. N. Y. Acad. Sci. 1085, pp. 197-207

Balaras, E. and Yang, J. (2005). Nonboundary Conforming Methods for LargeEddy Simulations of Biological Flows. J. of Fluid Engineering, 127, pp. 851-857.

Bluestein, D., Niu, L., Schoephoester, R. T., and Dewanjee, M. K. (1996). Steady flow in an aneurysm model: Correlation between fluid dynamics and blood platelet deposition. J. Biomechanical Engineering, 118(3), pp. 280-286.

Butty, V. D., Gudjonsson, K., Buchel, P., Makhijani, V. B., Ventikos, Y., and Poulikakos, D. (2002). Residence times and basins of attractions for realistic right internal carotid artery with two aneurysms. Biorhelogy, 39, pp. 387-393.

Canton, G., Levy, D., Lasheras, J. C., and Nelson, P. K. (2005). Flow changes caused by the sequential placement of stents across the neck of sidewall cerebral aneurysms. J. Neurosurgery, 103, pp. 891-902. 
Castro, M.A., Putman C. M., and Cebral, J. R. (2005). Applications of vascular CFD for clinical evaluation of cerebral aneurysms. Third Conference on Computational Fluid and Solid Mechanics, pp. 602-606.

Cebral, J.R., Castro, M.A., Appanaboyina, S., Putman, C.M., Millan, D., and Frangi, A.F. (2005). Efficient pipeline for image-based patient-specific analysis of cerebral aneurysm hemodynamic: Technique and sensitivity. IEEE trans. on Medical Imaging, 24, pp. 457-464.

Celik, I. B., 2004, "Procedure for Estimation and Reporting of Discretization Error in CFD Applications, ASME Journal of Fluids Engineering, Retrieved from http://www.asme.org/pubs/journals/fluideng/JFENumAccuracy.pdf, 2004

Chant, R. J. (2002). Secondary circulation in a region of flow curvature: Relationship with tidal forcing and river discharge, J.Geophys. Res., 107(C9), 3131, doi:10.1029/2001JC001082

Chandra K. B. (1992). Cardiovascular Biomechanics. New York University Press, New York.

Chintavis, S. M., Hademenos, G., and Powers, W. J. (1995). Hemodynamic assessment of the development and rupture of intracranial aneurysm using computational simulations. Neurology Research, 17(6), pp. 426-434.

Corcoran, T. E., Shortall, B. P., Kim, I. K., Meza, M. P., and Chigier, N. (2003). Aerosol Drug Delivery Using Heliox and Nebulizer Reservoirs: Results from an MRIBased Pediatric Model. J. Aerosol Medicine, 16, No. 3, pp. 263-271. 
Cussler, E. L. (1997). Diffusion Mass Transfer in Fluid System, 2nd ed. Cambridge University Press.

Dempere-Marco, L., Oubel, E., Castro, M., Putman, C., Frangi, A., and Cebral, J. (2006). CFD Analysis Incorporating the Influence of Wall Motion: Application to Intracranial Aneurysms. Proceedings of MICCAI'06, 9th International Conference, Copenhagen, Denmark, October 1-6.

Lee, D. H., Hur, S. H., Kim, H. G., Jung, S. M., Ryu, D. S., and Park, M. S. (2001). Treatment of Internal Carotid Artery Dissections with Endovascular Stent Placement: Report of Two Cases. Korean Journal of Radiology, 2001 March, Vol. 2(1), pp. $52-56$

Ersahin, C. (2007). Aerosol Generation and Entrainment Model for Cough Simulations. [Dissertation Defense]

Feng, Y., Wada, S., Tsubota, K., and Yamaguchi, T. (2004). Growth of Intracranial Aneurysms Arised from Curved Vessels under the Influence of Elevated Wall Shear Stress - A Computer Simulation Study. JSME International Journal, 47, pp. 1035-1042.

Fluent 6.2 User’s Guide. Fluent Inc., Lebanon, NH, 2005.

Ford, M. D., Hristo, N. N., Holdsworth, D. W., and Steinman D. A. (2003). The effect of gravity on cine X-Ray angiography. Summer Bioengineering Conference, Florida, USA, June 25-29

Foutrakis, G. N., Yonas, H., and Sclabassi, R. J. (1999). Saccular aneurysms formation in curved and bifurcating arteries. AJNR Am J Neuroradiol, 20, pp. 1309-1317. 
Funamoto, K., Hayase, T., Saijo, Y., and Yambe, T. (2007). Accuracy of Ultrasonic-Measurement-Integrated Simulation for three-dimensional Blood Flow in Aneurismal Aorta. Proceedings of $5^{\text {th }}$ Joint ASME/JSME Fluid Engineering Summer Conference, July 30-August 2, San Diego, FEDSM2007-37497.

Gambit 2.3.16 User's Guide. Fluent Inc., Lebanon, NH, 2006.

General Electric Healthcare of Visipaque (Iodixanol) Injection. [Material Safety Data Sheet]

Gonzales, C. F., Cho, Y. I., Ortega, H. V., and Moret, J. (1992). Intracranial aneurysms: Flow analysis of their origin and progress. AJNR Am. J. Neuroradiol, 13(1), pp. 181-188.

Gorden, C., Laudan, J., Gatchell, S., and Zeumer, H. (2001). Three-Dimensional pulsatile flow simulation before and after endovascular coil embolization of a terminal cerebral aneurysm. J. Cerebral Blood Flow \& Metabolism, 21, pp. 1464-1471.

Gray, Henry. (2000). Anatomy of the Human Body. Philadelphia: Lea \& Febiger, 1918; Bartleby.com, www.bartleby.com/107/. [August, 2007]

Harvey, W. (1628). On the Motion of the Heart and Blood in Animals. Prometheus Books, Buffalo, New York.

Hayakawa, M., Katada, K., Anno, H., Imizu, S., Hayashi, J., Irie, K., Negoro, M., Kato, Y., Kanno, T., and Sano, H. (2005). CT angiography with electrocardiographically gated reconstruction for visualizing pulsation of intracranial aneurysm: Identification aneurismal protuberance presumably associated with wall thinning. Am. J. Neuroradiol, 26, pp. 1366-1369. 
Himeno, M., Noda, S., and Himeno, R. (2007). Application of generic algorithm to prediction of artery geometric determinary. Proceedings of $5^{\text {th }}$ Joint ASME/JSME Fluid Engineering Summer Conference, July 30-August 2, San Diego, FEDSM200737241.

Hoi, Y., Meng, H., Woodward, S. H., Bendok, B. R., and Hanel, R. A. (2004). Effects of arterial geometry on aneurysm growth: three-dimensional computational fluid dynamic study. J. Neurosurgery, 99, pp. 676-81.

Hoshina, K., Sho, E., Sho, M., Nakahashi, T. K., and Dalman, R. L. (2003). Wall shear stress and strain modulate experimental aneurysm cellularity. J. Vascular Surgery, 37, pp. 1067-1074

Isida, F., Ogawa, H., Simizu, T., Kojima, T., and Taki, W. (2005). Visualizing the dynamics of cerebral aneurysms with four-dimensional computed tomographic angiography. Neurosurgery, 75 , pp. 460-471.

Isoda, H., Hirano, M., Takeda, H., Kosugi, T., Alley, M. T., Markl, M., Pelc, N. J., and Sakahara, H. (2006) Visualization of hemodynamics in a silicon aneurysm model using time-resolved, 3D, phase-contrast MRI. AJNR Am J. Neuroradiol, 27, pp. 11131118.

Jou, L., Wong, G., Dispensa, B., Lawton, M. T., Higashida, R. T., Young, W. L., and Saloner, D. (2005). Correlation between luminal geometry changes and hemodynamics in fusiform intracranial aneurysms. AJNR Am J. Neuroradiol, 26, pp. 2357-2363. 
Jou, L-D., Quick, C. M., Young, W. L., Lawton, M. T., Higashida, R., Martin, A., and Saloner, D. (2003). Computational Approach to Quantifying Hemodynamic Forces in Giant Cerebral Aneurysms. AJNR Am J. Neuroradiol, 24, pp. 1804-1810.

Józefowicz, R. F., Miller, J. J, and Powers, J. M. (2000). Neuropathology and Neuroimaging Laboratory: Mind, Brain and Behavior Course. Departments of Pathology and Neurology, University of Rochester, Available at http://www.urmc.rochester.edu/neuroslides/index.html

Kang, H. S., Oh, C.W., Han, M. H., Byun, H. S., and Han, D. H. (2005). Treatment of a Sequential Giant Fusiform Aneurysm of the Basilar Trunk. Korean Journal of Radiology, June; Vol. 6(2), pp. 125-129

Karmonik, C., Arat, A., Benndorf, G., Akpek, S., Klucznik, R., Mawad, M. E., and Strother, C. M. (2004). A technique for improved quantitative characterization of intracranial aneurysms. AJNR Am J. Neuroradiol, 25, pp. 1158-1161.

Kassell, N.F. and Torner, J.C. (1982). Epidemiology of intracranial aneurysms. Int. Anethesiol Clin., 20, pp. 13-17.

Kim, C., Cervos-Navarro, J., Kikuchi, H., Hashimoto, N., and Hazama, F. (1992b). Alterations in Cerebral Vessels in Experimental Animals and their Possible Relationship to the Development to the Aneurysms. Surg. Neurol., 38, pp. 331-337

Kim, C., Cervos-Navarro, J., Patzold, C., Tokuriki, Y., Takabe, Y., and Hori, K. (1992a). In Vivo Study of Flow Pattern at Human Carotid Bifurcation with Regard to Aneurysm Development. Acta Neurochir. (Wien), 115, pp. 112-117. 
Kondo, S., Hashimoto, N., Kikuchi, H., Hazama, F., Nagata, I., and Kataoka, H. (1997). Cerebral aneurysms arising at nonbranching sites. An experimental study. Stroke. 28, pp. 398-403; discussion pp. 403-404.

Krings, T., Moller-Hatmann, W., Hans, F. J., Thiex, R., Brunn, A., Scherer, K., Meetz, A., Dreeskamp, H., Stein, K. P., Gilsbach, J. M., and Thron, A. (2003). A refined method for creating saccular aneurysms in the rabbit. $J$. Neuroradiology, 45, No.7, pp. 423-429.

Ku, D. N., Giddens, D. P., Phillips, D. J., and Strandness, D. E. (1985). Hemodynamics of the normal human carotid bifurcation: in vitro and in vivo studies. Ultrasound Med Biol., 11, pp. 13-26.

Levick, J.R., (2003). An Introduction to Cardiovascular Physiology. Oxford University Press Inc.

Lieber, B.B., and Giddens, D. P. (1990). Post-Stenotic Core Flow Behavior in Pulsatile Flow and its Effects on Wall Shear Stress. J. Biomech., 23, pp. 597-605.

Malek, A.M, Alper, S.L., Izumo, S. (1999). Hemodynamic shear stress and its role in atherosclerosis. JAMA., 282, pp. 2035-2042.

Mantha, A., Karmonik, C., Benndorf, G., Strother, C., and Metcalfe, R. (2006). Hemodynamics in a Cerebral Artery before and after the Formation of an Aneurysm. AJNR Am J Neuroradiol, 27, pp. 1119-1122.

Martindale. (1996). The Extra Pharmacopoeia, 31st Ed., Pharmaceutical Press, pp. 1011.

Mchedlishvili, G. I. (1986). Arterial Behavior and Blood Circulation in the Brain. New York 
Meng, H., Wang, Z., Kim, M., Ecker, R. D., and Hopkins L. N. (2006). Saccular aneurysms on straight and curved vessels are subject to different hemodynamics: Implications of intravascular stenting. AJNR Am J Neuroradiol, 27, pp. 1861-1865.

Metcalfe, R. W. (2003). The Promise of Computational Fluid Dynamics as a Tool for Delineating Therapeutic Options in the Treatment of Aneurysms. AJNR Am J. Neuroradiol, 24, pp. 553-554.

Miller, R., Battaglia, F., and Olsen, M. G. (2005). A Computational and Experimental Investigation of Flow in an Intracranial Side-Wall Aneurysm. Proceedings of MICCAI'06, 9th International Conference, Houston, USA, June 19-23.

Miniño A. M., Heron M. P., and Smith B. L. (2006). Deaths: Preliminary Data for 2004. National vital statistics reports; Vol. 54 no. 19. Hyattsville, MD: National Center for Health Statistics.

Mittal, R., Simmons, S. P., and Udaykumar, H. S. (2001). Application of LargeEddy Simulation to the Study of Pulsatile Flow in a Modeled Arterial Stenosis. $J$. Biomech. Eng., 123, pp. 325-332.

Nanduri, J. R., Pino-Romainville, F. A., and Celik, I. B. (2007). Mesh Generation for CFD of Biological Flows: Internal and External Flow Meshes from Diagnostic Images. FLUCOME'07, 9th International Conference on Fluid Control, Measurement and Visualization, September 16-19.

Pardridge, W. M., (1998). Introduction to the Blood-Brain Barrier: Methodology, Biology and Pathology. $1^{\text {st }}$ Ed., Cambridge University Press. 
Pedley, T. J. (1977). Pulmonary fluid dynamics. Annu. Rev. Fluid Mech. 9, pp. 229-274.

Pedley, T. J. (1980). The Fluid Mechanics of Large Blood Vessels. Cambridge University Press.

Perktold, K. and Rappitsch, G. (1995). Computer simulation of local blood and vessel mechanics in a compliant carotid artery bifurcation model. J. Biomech, 28, pp. 845-856.

Pino-Romainville, F. A., Nanduri, J. R., Rai, A., and Celik, I. B. (2007). Dispersion Study in a Giant Intracranial Aneurysm using Computational Fluid Dynamics Techniques. Proceedings of $5^{\text {th }}$ Joint ASME/JSME Fluid Engineering Summer Conference, July 30-August 2, San Diego, FEDSM2007-37444.

Plesniak, M.W. and Peterson, S.D. (2007). Keynote Paper: Biomedical Fluid Dynamics - Rich Flow Physics in Pulsatile Flow. Proceedings of $5^{\text {th }}$ Joint ASME/JSME Fluid Engineering Summer Conference, July 30-August 2, San Diego, FEDSM200737342

Prakash, S. and Ethier, C. R. (2001). Requirements for Mesh Resolution in 3D Computational Hemodynamics. Transactions of ASME, 123, pp. 134-144

Rai, A. (2006-2007). Private Communications

Raymond, J.; Roy, D., Leblanc, P., Roorda, S., Jannicki, C., Normandeau, L., Morel, F., Gevry, G., Bahary, J. P., Chagnon, M., Guilbert, F., and Weill, A. (2003). Endovascular Treatment of Intracranial Aneurysms with Radioactive Coils: Initial Clinical Experience. Stroke, 34, pp. 2801-2806 
Ramalinga S. D., Lloyd, D. P., and Vincent, T. T. (1997). Hemodynamics of Intracranial Lateral Aneurysms: Flow Simulation Studies. Biomedical Engineering Conferences. Proceedings of the 1997 Sixteenth Southern, 24, pp. 224-227.

Reid, R. C., Sherwood, T. K., and Prausnitz, J. M. (1977). Properties of Gases and Liquids, 3rd Ed. Ed. McGraw-Hill.

Scheinberg, P., and Joyne, H. W. (1952). Factors Influencing Cerebral Blood Flow and Metabolism. Circulation, 5, pp. 225-236

Schwuchow, J. M., Kern, V. D., and Sack, F. D. (2002). Tip-Growing Cells of the Moss Ceratodon purpureus are Gravitropic in High-Density Media. Plant Physiology, 130, pp. 2095-2100.

Scotti, A., and Piomelli, U. (2001). Numerical Simulation of Pulsatile Turbulent Channel Flow. Phys. Fluids, 13, 5, pp. 1367-1384.

Shandas, R., Kim, H-B., Hertzberg, J. R., and Mukdadi, O. (2003). An Ultrasound Base Method for Measuring Multiple Velocity Components in Opaque Macro and Micro Flows. Transducers'03, $12^{\text {th }}$ International Conference on Solid State Sensors, Actuators and Microsystems, Boston, June 8-12.

Shojima, M., Oshima, M., Takagi, K., Torii, R., Hayakawa, M., Katada, K., Morita, A., and Kirino, T. (2004). Magnitude and role of wall shear stress on cerebral aneurysms: Computational Fluid Dynamic study of 20 middle cerebral aneurysms. Stroke, 35, pp. 2500-2505.

Stehbens, W. E. (1989). Etiology of intracranial berry aneurysm. J. Neurosurgery, 70, pp. 823-831. 
Steiger, H. J. (1990). Pathophysiology of development and rupture of cerebral aneurysms. Acta Neurochir Suppl. (Wien), 48, pp. 1-57.

Steinman, D. A., Milner, J. S., Norley, C. J., Stephen, P. L., and Holdsworth, D. W. (2003). Image-Based Computational Simulation of Flow Dynamics in a Giant Intracranial Aneurysm. AJNR Am J. Neuroradiol, 24, pp. 559-566.

Stroud, J.S., Berger, S.A., and Saloner, D. (2002). Numerical Analysis of Flow through a Severely Stenotic Carotid Artery Bifurcation. ASME J. Biomech. Eng., 102, pp. $9-20$.

Taylor, C. (2008). Private communications.

Tittelbaugh, E. M., Fu, R., and Sett, S. (2007). Coupling FEA to CFD to Investigate the Effects of Pulsatile Blood Flow on the Dilatation of Artery Walls. www.ensight.com/images/stories/pdf/NWC07-tittelbaugh-fusett_abaqus_fluent_fsi_nafems_2007_paper.pdf, last accessed on March $17^{\text {th }} 2007$

Toth, M., Nadasy, G. L., Nyar, I., Kerenyi, T., and Monos, E. (2003). Are there systematic changes in the arterial biomechanics of intracranial aneurysm patients? Pflugers Arc., 439, pp. 573-578.

Utter, B., and Rossmann, J. S. (2006). Influence of Shape on Saccular Aneurysm Hemodynamics and Risk of Rupture. Bioengineering Conference Proceeding of IEEE 32nd annual, pp. 21-22.

Weir, B., Amidei, C., Kongable, G., Findlay, J. M., Kassel, N. F., Kelly, N., Dai, L., and Karrison, T. G. (2003). The aspect ratio (dome/neck) of rupture and unruptured aneurysms. J. Neurosurgery, 99, pp. 447-451. 
Wilson, K., Whyman, M., Hoskins, P., Lee, A. J., Bradbury, A. W., Fowkes, F. G., Ruckley, C. V. (1999). The relationship between abdominal aortic aneurysm wall compliance, maximum diameter and growth rate. Cardiovasc Surg., 7, pp. 208-213.

Yang, J. and Balaras, E. (2006). An embedded-boundary formulation for largeeddy simulation of turbulent flows interacting with moving boundaries. $J$. of Computational Physics, 215, pp. 12-40

Younis, B. A. and Berger, S. A. (2004). A Turbulence Model for Pulsatile Arterial Flows. Transactions of ASME J Biomedical Engineering, Vol. 126, pp. 578-584.

Zarins, C. K., Giddens, D. P., Bharadvaj, B. K., Sottiurai, V. S., Mabon, R. F., and Glagov, S. (1983). Carotid bifurcation atherosclerosis. Quantitative correlation of plaque localization with flow velocity profiles and wall shear stress. Circulation Research, 53, pp. 502-14

Zeller, H., Talukder, N., and Lorenz, J. (1970). Model studies of pulsating flow in arterial branches and wave propagation in blood vessels. In Fluid Dynamics of Blood Circulation and Respiratory Flow, AGARD Conference Proceedings, 65. 


\section{Appendix A: Geometry Reconstruction Validation}

The code that was used to reconstruct the aneurysm geometry is validated by rebuilding a well known geometry. This geometry corresponds to a child's upper respiratory track from Corcoran's investigation used in the study “Aerosol Drug Delivery Using Heliox and Nebulizer Reservoirs: Results from an MRI-Based Pediatric Model". A comparison of the dimensions of the model constructed by Corcoran et al (2003), and those reconstructed using our procedure is given in Table A1.

The result shows good agreement with the reference values. This reconstruction process is better than the Corcoran performed because Corcoran's reconstruction differs about $\pm 6 \%$ from the reference values. On the other hand, the results obtained by the MATLAB code software differ less than $\pm 5 \%$ from the reference values.

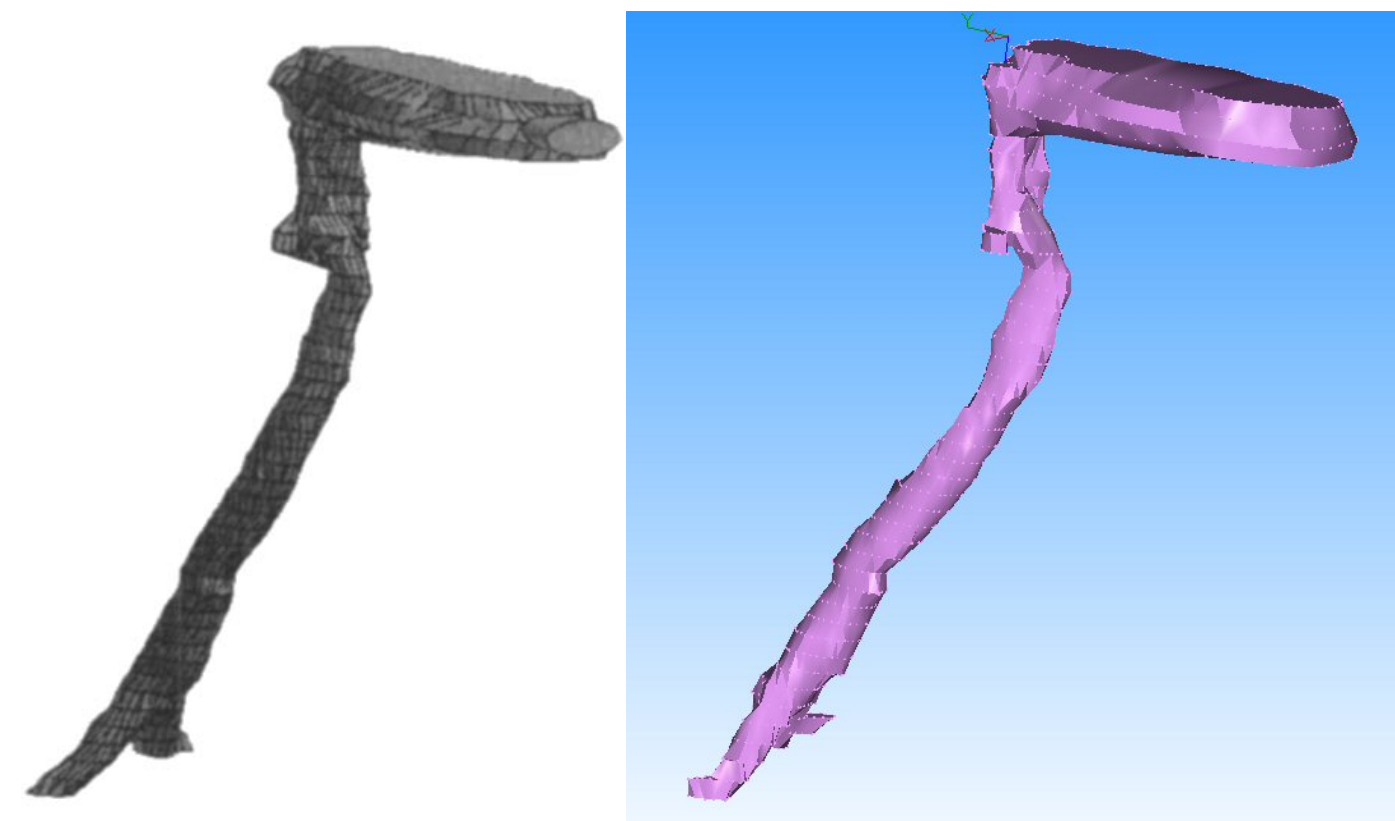

Figure A1: Geometry reconstruction comparison between Corcoran's procedure and our procedure 
Table A1: Dimensions of the Reconstructed Model Compared to Corcoran's References

\begin{tabular}{|c|c|c|c|c|}
\hline \multicolumn{5}{|c|}{ Reierences } \\
\hline $\begin{array}{l}\text { Parameter of } \\
\text { comparison }\end{array}$ & $\begin{array}{c}\text { Source of } \\
\text { measurement }\end{array}$ & $\begin{array}{l}\text { Values } \\
\text { from } \\
\text { reference } \\
\end{array}$ & $\begin{array}{c}\text { Values from } \\
\text { Corcoran's } \\
\text { model } \\
\end{array}$ & $\begin{array}{c}\text { Values } \\
\text { from our } \\
\text { model } \\
\end{array}$ \\
\hline $\begin{array}{l}\text { Width of dental } \\
\text { arch maxilla } \\
(\mathrm{mm})\end{array}$ & $\begin{array}{l}\text { Correlation by } \\
\text { age and sex }\end{array}$ & 29 & 33 & 31 \\
\hline $\begin{array}{l}\text { Length of } \\
\text { dental arch } \\
\text { maxilla (mm) }\end{array}$ & $\begin{array}{l}\text { Correlation by } \\
\text { age and sex }\end{array}$ & 29.5 & 28 & 29 \\
\hline $\begin{array}{l}\text { Vocal tract } \\
\text { length }(\mathrm{mm})\end{array}$ & $\begin{array}{l}\text { MRI-both sexes } \\
(\mathrm{n}=9, \text { ages } 5 \\
\text { and } 6)\end{array}$ & $\begin{array}{l}99.2 \\
(91.4- \\
110.2)\end{array}$ & 118.0 & - \\
\hline $\begin{array}{c}\text { Oropharyngeal } \\
\text { distance }(\mathrm{cm})\end{array}$ & $\begin{array}{l}\text { MRI correlation } \\
\text { by age based on } \\
100 \text { subjects }\end{array}$ & 7.0 & 6.4 & $6.4^{*}$ \\
\hline $\begin{array}{l}\text { Glottal length } \\
\quad(\mathrm{mm})\end{array}$ & $\begin{array}{l}\text { Cadavers-male } \\
(\mathrm{n}=5 \text {, ages } 2-5)\end{array}$ & 10.3 & 9.0 & 8.6 \\
\hline $\begin{array}{l}\text { Glottal length } \\
\quad(\mathrm{mm})\end{array}$ & $\begin{array}{l}\text { Radiology CT } \\
(n=5, \text { ages } 2-6)\end{array}$ & $8-10$ & 9.0 & 8.6 \\
\hline $\begin{array}{l}\text { Subglottic } \\
(6 \mathrm{~mm}) \text { ant- } \\
\text { post length }\end{array}$ & $\begin{array}{l}\text { Radiology CT } \\
(n=5, \text { ages } 2-6)\end{array}$ & $7-10$ & 8.5 & - \\
\hline $\begin{array}{l}\text { Tracheal length } \\
\text { (cm) }\end{array}$ & $\begin{array}{l}\text { Empirical } \\
\text { model }\end{array}$ & 7.4 & 7.8 & $7.8^{*}$ \\
\hline $\begin{array}{c}\text { Tracheal } \\
\text { diameter }(\mathrm{mm})\end{array}$ & $\begin{array}{l}\text { Empirical } \\
\text { model }\end{array}$ & 9.3 & $9.9 \mathrm{a}$ & 8.1 \\
\hline $\begin{array}{c}\text { Tracheal } \\
\text { diameter (mm) }\end{array}$ & $\begin{array}{l}\text { Radiology CT } \\
(n=5, \text { ages } 2-6)\end{array}$ & $7-10$ & $9.9 \mathrm{a}$ & 8.1 \\
\hline $\begin{array}{c}\text { Mainstem } \\
\text { bronchial } \\
\text { diameter }(\mathrm{mm})\end{array}$ & $\begin{array}{l}\text { Empirical } \\
\text { model }\end{array}$ & 8.1 & R 7.5/L 8.0 & R 7.8/L 8.2 \\
\hline
\end{tabular}


Figure A2 shows the upper respiratory track successfully imported in Gambit, where it can be re-meshed and define to the boundary conditions. The green lines located in the upper region of the image correspond to the mouth. This geometry has been modified in 3DReshaper by removing the secondary bronchi (bifurcation region). Only the primary bronchi is needed to simulate the coughing cycle.

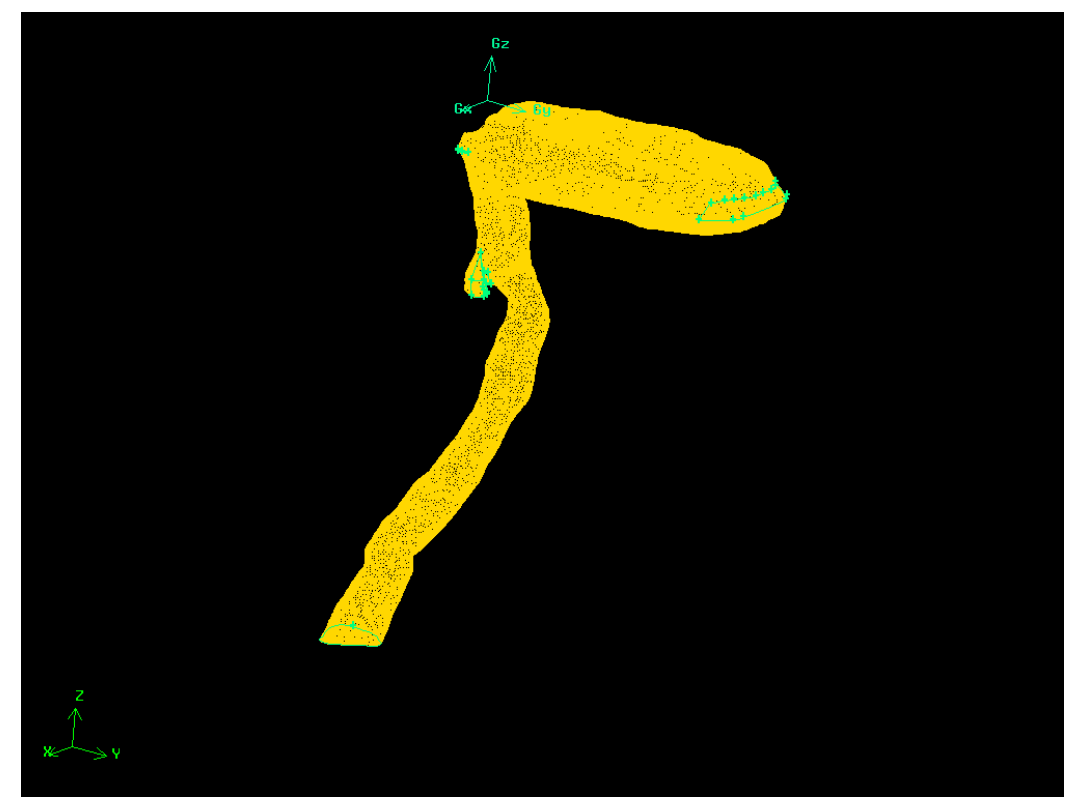

Figure A2: Upper respiratory track geometry imported in Gambit

Similar to the cardiac cycle imposed at the parental inlet of the aneurismal geometries, a coughing waveform (Ersahin, 2007) is imposed at the primary bronchi. This simulation was carried out using $\kappa-\varepsilon$ turbulent model due to the nature of the air flow characteristics. Preliminary results are shown in Figure A3 at the coughing peak; this simulation was performed only to corroborate the upper respiratory track reconstruction and use. This figure also shows the z-velocity colored by the magnitude in the geometry and the velocity vectors. The parameter for this simulation was only one phase flow (air) which the flowrate peak is at $\mathrm{t}=0.1 \mathrm{sec}$. Latter time is also recorded 
(beginning of the deceleration process) $(\mathrm{t}=0.15 \mathrm{sec})$ and the flow patterns is described by velocity vectors through the geometry. Hence, the geometry reconstruction using $2 \mathrm{D}$ images using the MatLab program developed "in house" is validated.

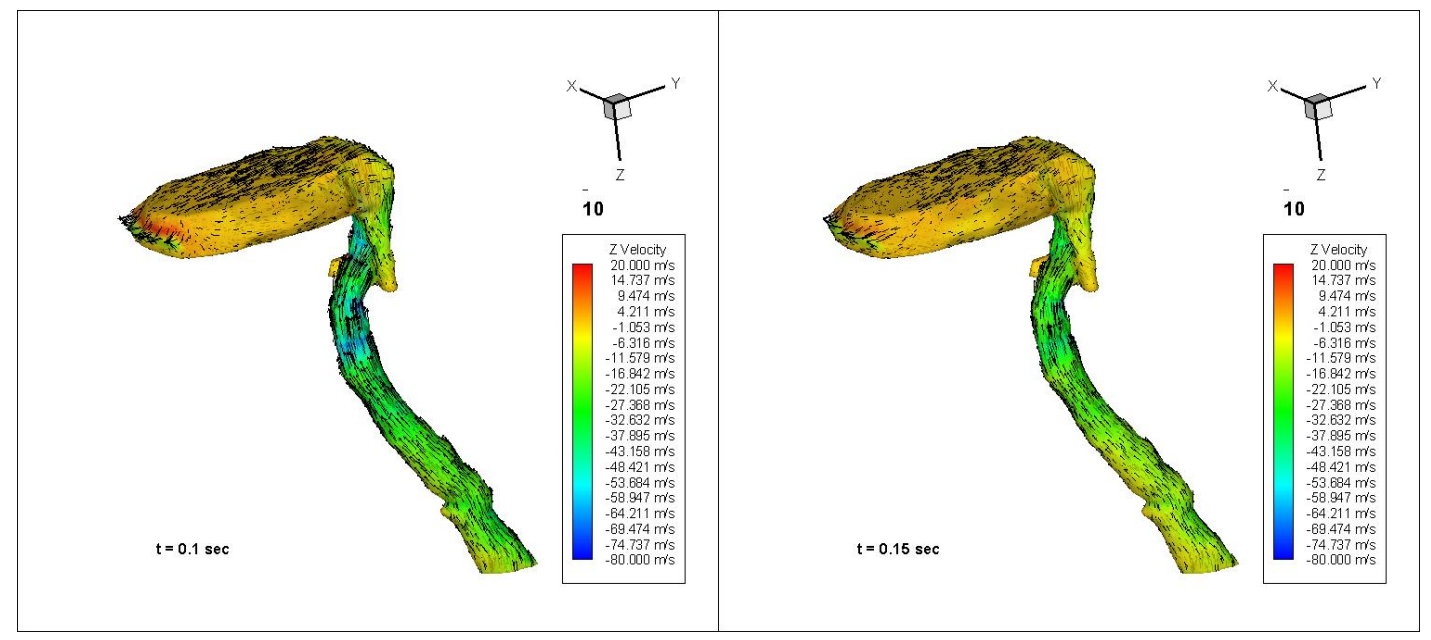

Figure A3: Velocity vectors colored by the $\mathrm{z}$ velocity in the upper respiratory track during the coughing peak (left) and coughing decelerating phase (right) 


\section{Appendix B: Experimental Investigation}

\section{Experimental Set-up and Apparatus}

Laboratory experiments are performed with a bifurcating pipe in order to corroborate the numerical simulation. The Y-pipe model is constructed by using acrylic plastic with a similar diameter as the arteries. The same notations are used for the cross sections parental inlet (PI), large outlet $(\mathrm{O} 1)$, and small outlet $(\mathrm{O} 2)$ measuring $5 \mathrm{~mm}, 4$ $\mathrm{mm}$, and $3 \mathrm{~mm}$ respectively. Both child outlets $\mathrm{O} 1$ and $\mathrm{O} 2$ have an angle of 45 degrees with respect to a projection of PI cross section. The length of the bifurcation model is 6.5 $\mathrm{cm}$ which only the PI measures $4 \mathrm{~cm}$. Figure B1 shows the Y-pipe model used in the experimental set-up. This Figure also shows that the outlets have marked with different colors to be distinguished during the experiment.

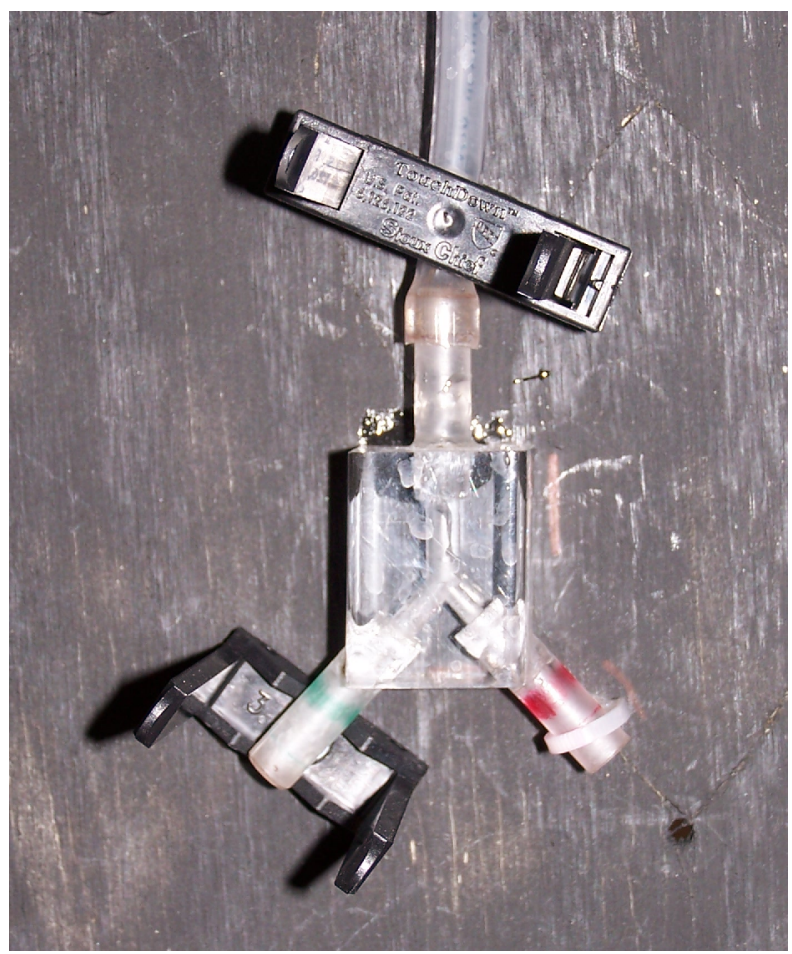

Figure B1: Y-pipe close-up used in the experimental set-up 
The red and green colors correspond to $\mathrm{O} 1$ and $\mathrm{O} 2$ respectively. To avoid the movement of the bifurcation model, which can cause variations in the final results, the bifurcation model is leveled and glued to a panel. In addition, the rubber pipes are joined to the three cross sections and fixed to the panel. The ending cross sections of rubber pipes (PI, O1, and $\mathrm{O} 2$ ) are sealed with epoxy, the Y-pipe as a preventive mode to water losses, thus the mass transport is conserved during the experiment.

Figure B2 shows the complete experimental set-up. In this Figure, a rotameter is placed in the upstream direction of the bifurcation model to measure the instantaneous volumetric flow. Before this apparatus is placed, it is calibrated to assure accurate reading measurement. In the downstream direction of the Y-pipe, a beaker is placed at the $\mathrm{O} 2$ outlet to quantify the accumulated volumetric flow discharge during the experiment. The O1 outlet is drained. Additional apparatus and accessories are used, such as a stop watch, a video camera, and a column of water. The stop watch and the video camera are utilized to keep track of the time and to record the reading of the instantaneous volumetric flow given by the flowmeter. The column of water would serve as a reservoir to maintain constant water fluid flow during the experiment.

\section{Experimental Procedure}

The rotameter is initially closed while the column of water is filled to avoid air bubbles getting into the system. Once the water column is filled, the rotameter is opened to yield the water flow through the system. The velocity depends on the accumulated water column weight, the PI diameter, and the gravitational force. Every $20 \mathrm{~mL}$ reading changes from the beaker, and measurements are taken simultaneously from the flowmeter 
until the column of water is totally discharged. All the experiments recorded by the camera focused the beaker and the rotameter. The obtained data is corroborated by watching the video. Every experiment is recorded and the data obtained is confirmed by this process. The data obtained during the experiments exhibit very similar results and they are averaged as it is shown in Figure B4. The Reynolds number is calculated and oscillates from 1,700 to 1,200 , with respect to PI cross section. Another dimensionless number (Womersley number) is calculated to determine the ratio of pulsatile flow frequency to the viscous effects being of 0.05 . This value is small due to the long time period of the experiment.

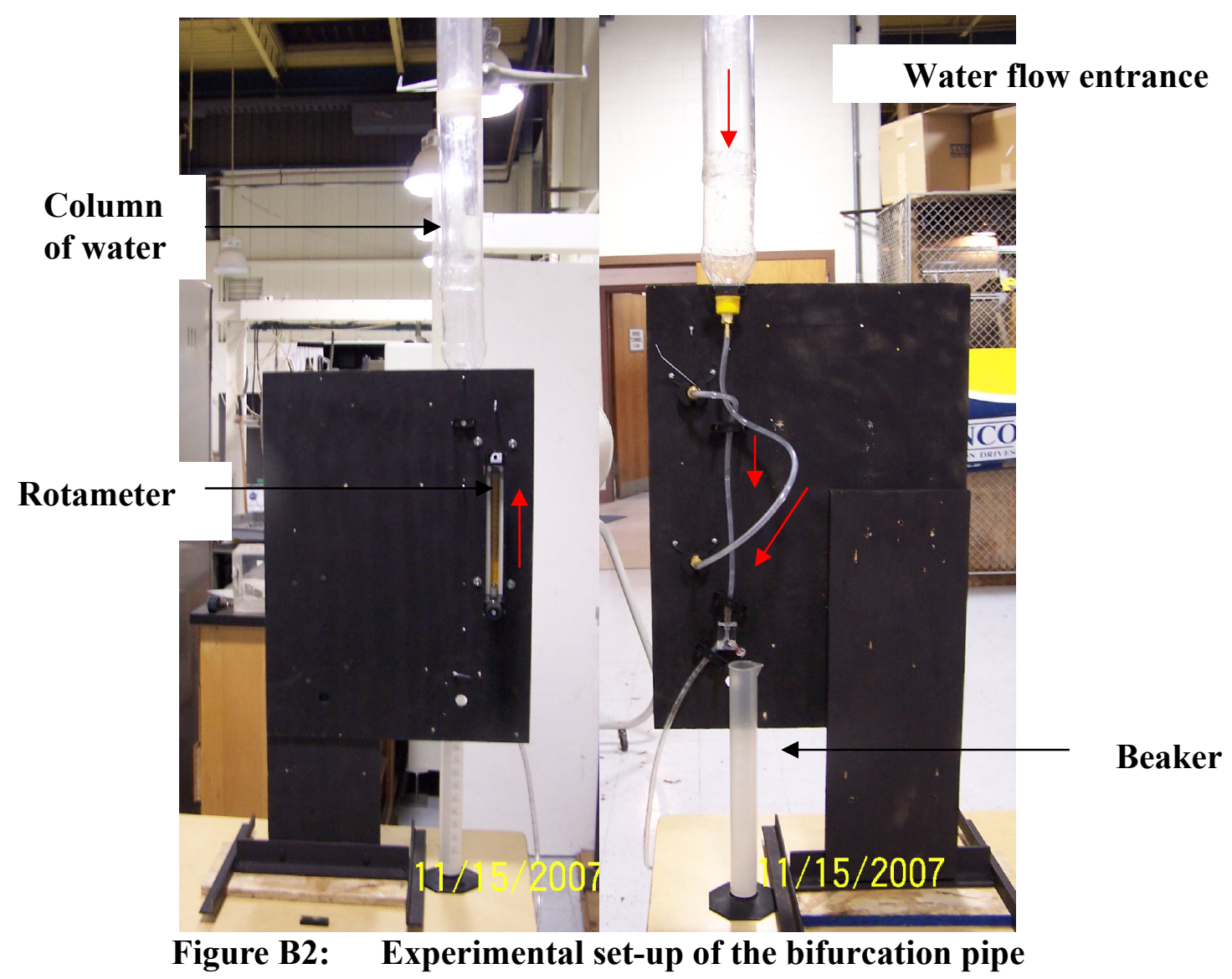




\section{Numerical Study}

Numerical simulations are performed to validate the experimental results using a similar geometry. The geometry has the same pipe diameters and the projection angles of the child pipes as the bifurcation model shown in Figure B3. The model is meshed using tetrahedral grids having 296,031 computational cell elements and the BC are defined. Pressure outlet $\mathrm{BC}$ and velocity inlet are chosen for the dual outlets and the inlet respectively. The volumetric flow rate obtained during the experiment is adjusted to a mathematical function and imposed at PI cross section of the y-pipe model. The fluid assumed in the simulation is water (incompressible Newtonian fluid). The equations are discretized using a second order scheme to carry out the numerical simulation with a convergence error of $10^{-3}$. Even though the total period of the experiments is about 180 seconds as shown in Figure B4, the simulation is performed for a period of 50 seconds.

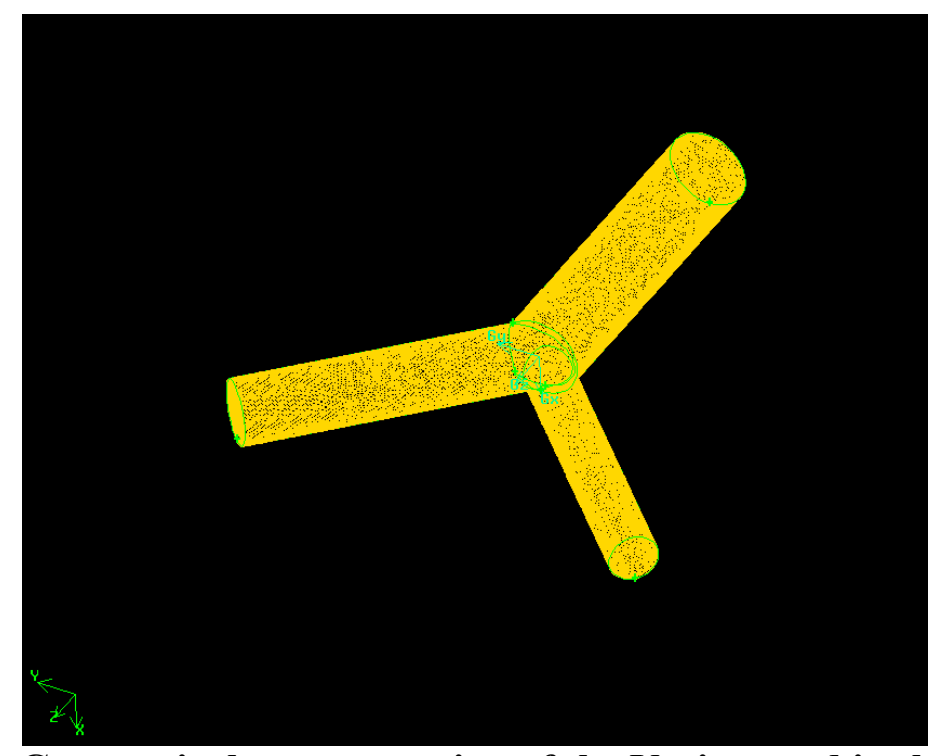

Figure B3: Geometrical representation of the Y-pipe used in the simulation 


\section{Results and Discussion}

Once the simulation process is completed, the volumetric flow rate at the outlets are plotted to be compared with the experimental results. Figure B4 shows the volumetric flow rate comparison of both of the outlets between the numerical simulations and the experiments. Due to the nature of the experiment, only the deceleration phase is carried out because of the importance of the reflected flow wave. Such flow reversal has an effect in the upstream region of the bifurcation during the deceleration phase. The results obtained in the numerical simulation and the experiments have a strong correlation in the prediction of the volumetric flow rate at the outlets. Overall, the results differ about $\pm 3 \%$. Figure B4 also shows an empty region for the experimental results $(75<\mathrm{t}<100)$, which it is due to the necessary time taken to discharge the filled beaker during the experiment. The small beaker is used in the current investigation in order to measure as accurately as possible the accumulated volumetric flow at $\mathrm{O} 2$. The discharge of the water from the beaker only takes few seconds to be resettled at the experimental set-up.

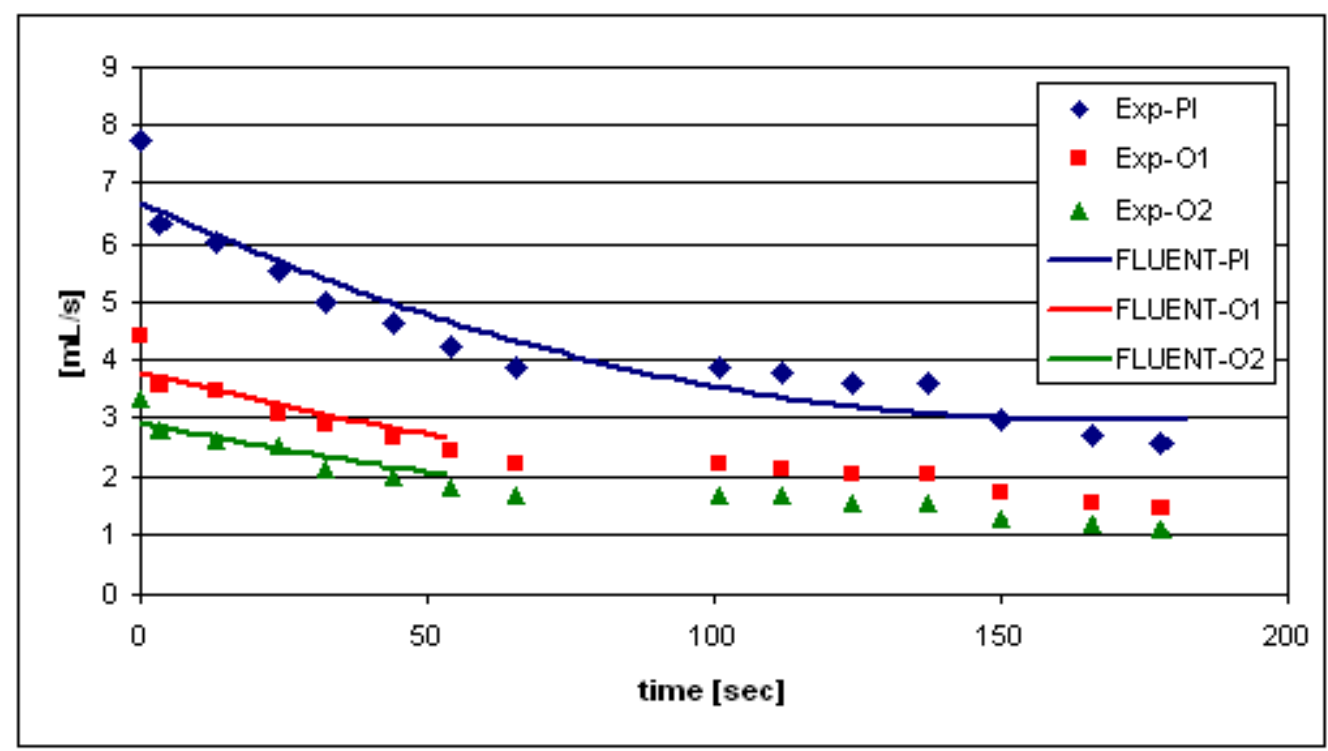

Figure B4: Volumetric flow rate comparison between the experiments and the numerical simulations 
The experiment is also performed for the acceleration phase; however, air bubbles are introduced to the system during the water pouring. The air bubbles affect not only the mass flow rate at each outlet, but they also block the small outlet during the experiment. In order to solve this problem, a computer-controlled flow system can be used to emulate repeatedly a defined volumetric waveform at the inlet like the cardiac cycle. Therefore, the water flow feeding would avoid the air bubbles' generation, assuring a continuous flow during the experiment. The high price of this system is the main reason why it was not used in the experimental set-up. An alternative method to imitate the cardiac cycle is to use a controlled manual pump, but it requires more a sophisticated experimental apparatus due to the drastic change of the volumetric flow measured by the rotameter and the beaker.

The advantage of numerical simulations is that the flow characteristics can be studied at any location of the geometry. Therefore, a symmetric plane is made to visualize the fluid flow at the bifurcation region, as shown in Figure B5. Recirculation flows are observed for both child tubes at the outer walls of the bifurcations. When the fluid moves downstream toward the bifurcation zone at high velocity $(\operatorname{Re}=1700)$, the flow tends to keep the point of maximum axial velocity closer to the inner side of the wall (junction region). On the other hand, the maximum axial velocity is found near by the outer side of the walls when the fluid moves at slower velocities $(\mathrm{Re}=1200)$. Hence the mass flow rate at the outlets would be affected by the fluid flow velocity. Additionally, the bifurcation sharpness plays an important role in the mass flow ratio at the outlets because it can determine the fluid flow behavior at this region. Pedley had 
carried out several experiments with different bifurcation sharpness and different projection angles of the child outlets (Pedley, 1977).

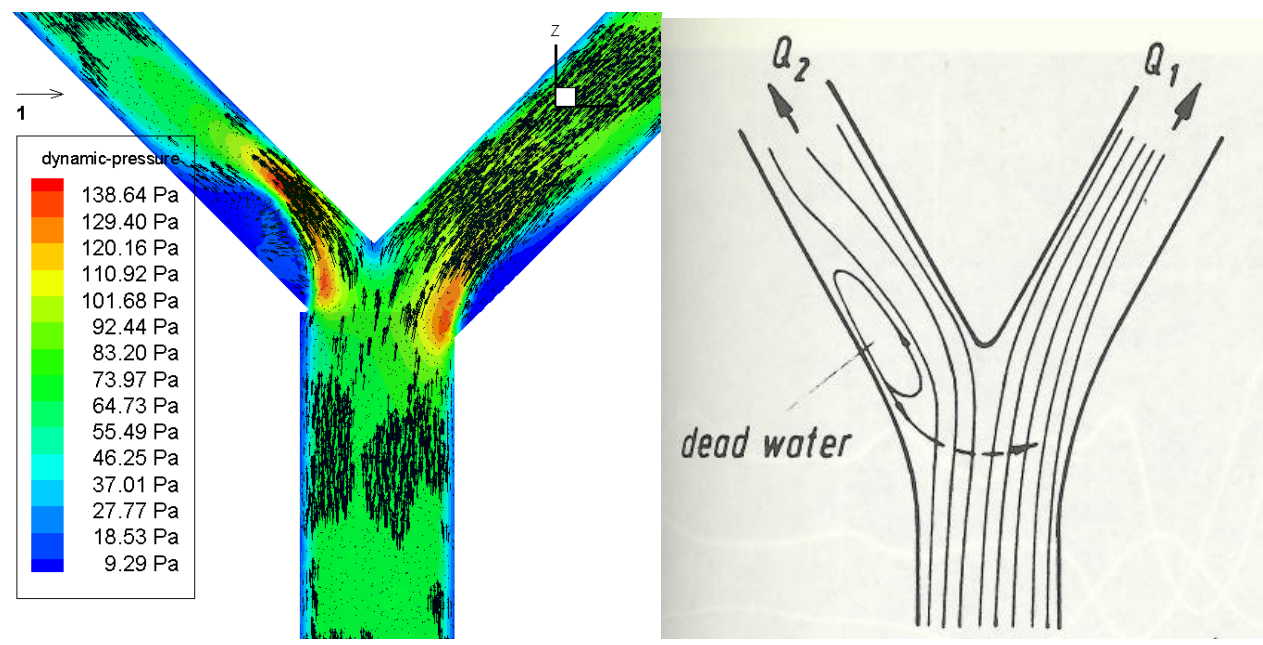

Figure B5: Qualitative comparison results of the fluid flow characteristics for the bifurcation model: Velocity vectors colored by the axial velocity at $\operatorname{Re}=1,200$ (right) and $\operatorname{Re}=1,100$ (left) (Zeller et al, 1970)

Complementary to Pedley's investigation, Zeller et al (1970) has observed that the separation and flow reversal at the outer wall is higher at the small outlet. These experimental results agree with the results shown in Figure B5. They also state that flow reversal (dead-water-zone) changes according to the volumetric flow imposed at the inlet and an increment of the flow pulsation raises the dead water rotation, increasing the shearing stress at the walls. The current simulation also agrees with Zeller's investigation and it is shown in Figure B6. In order to manipulate the volumetric flow, Zeller et al (1970) used a motor and a pulse generator valve to cause variable flow rates likewise the cardiac cycle. Figure B7 shows the pulse form and flow fluctuation used for Zeller et al's investigation, which can be compared qualitatively with the results obtained for the aneurysm geometries. The waveform used for cerebral aneurysm exhibits a simpler volumetric flow and the cardiac cycle period is 0.7 seconds. 


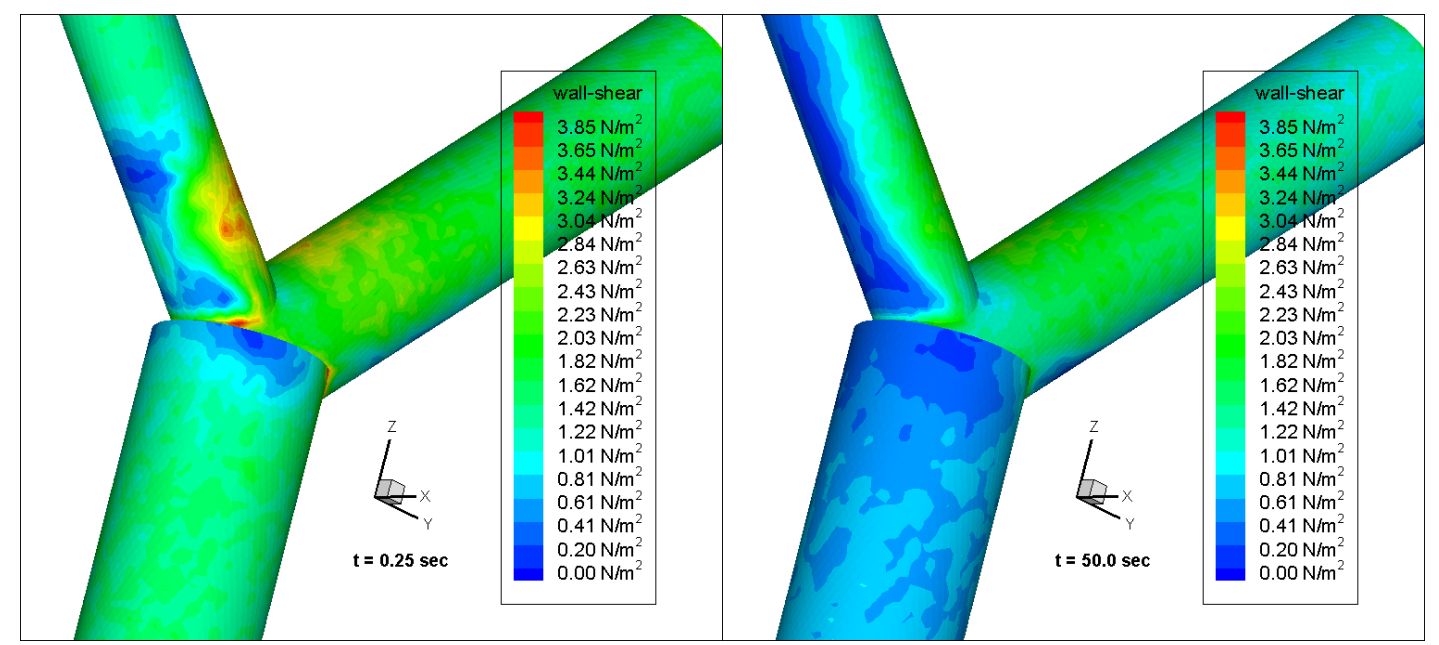

Figure B6: WSS contours of the bifurcation model: $R e=1,700$ (left) and $R e=1,200$ (right)

Different geometric configurations were used by Zeller et al in the experiments: a) same outflows diameters $\left.\left.\left(\mathrm{A}_{1}=\mathrm{A}_{2}\right) ; \mathrm{b}\right) \mathrm{A}_{1}+\mathrm{A}_{2}=1.2 \mathrm{~A}_{0} ; \mathrm{c}\right)$ different angles for the child outlets (i) $\theta_{1}=\theta_{2}=15$, (ii) $\theta_{1}=\theta_{2}=30$, and (iii) $\theta_{1}=14.8$ and $\theta_{2}=30$.

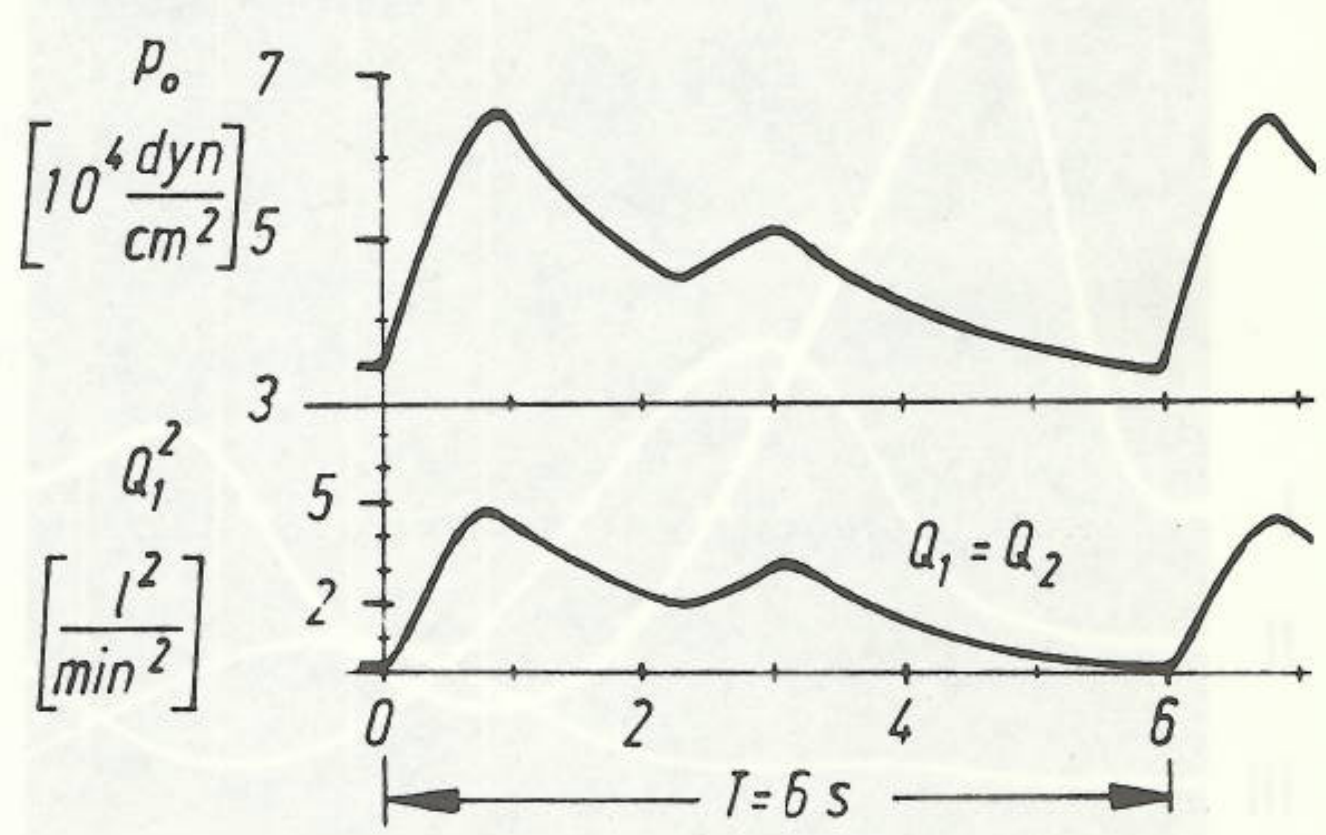

Figure B7: Pulse form and flow fluctuation (Zeller et al, 1970) 